Copyright

by

Sibi Mathew

2010 
The thesis committee for Sibi Mathew

Certifies that this is the approved version of the following thesis

\section{Evaluation of CFD Predictions Using Thermal Field Measurements on a Simulated Film Cooled Turbine Blade Leading Edge}

Approved by Supervising Committee:

David G. Bogard, Supervisor

Silvia Ravelli 


\title{
Evaluation of CFD Predictions Using Thermal Field Measurements on a Simulated Film Cooled Turbine Blade Leading Edge \\ by
}

Sibi Mathew, B.S

\author{
Thesis \\ Presented to the Faculty of the Graduate School of \\ The University of Texas at Austin \\ in Partial Fulfillment \\ of the Requirements \\ for the Degree of
}

Master of Science in Engineering

The University of Texas at Austin

December 2010 


\section{Dedication}

To my grandfather, the smartest man I know 


\section{Acknowledgements}

Firstly, I would like to thank Dr Bogard for his support and advice throughout the course of my graduate school career. He has been a great teacher and gave me the confidence to transform ideas into reality. I would also like to thank Dr Silvia Ravelli for all her support in helping me better understand CFD. Also, thank you for reviewing my thesis. I would also like to thank Mark Phillips for helping me with the stepper motor controller.

Furthermore, I would like to thank Jason Albert and Todd Davidson for helping me throughout the course of my experiments. I would also like to thank Laurene Dobrowolski and Gwennaël Chartrel for keeping me company in my CFD adventures. Thanks also to Jason Dees, Tom Dyson, Emily Pfautsch, Antoine and Randy Williams for making the TTCRL a more fun place. Special thanks go out to Claire Florette.

My closest friend, Kushal Kapse ,from using a restroom plunger for an engineering project to saving my life in New Orleans, thank you for everything. And, for making me the grasshopper I am now, the greatest of thanks to Terri Branham. To round up the best of friends, thanks also to Adam Namal, Arun Chauhan and Steve 'Atlantic Ocean' Mani. Also, my gratitude to my roommates, Art, Rudy and Baldemar. Heres to the good times at the Gingerman,

I would like to thank my Uncle, Dr Narayan Hedge and my Aunt, Kusum Hegde for their support throughout the years. And to my parents, K.D Mathew and Ani Alexander, and my grandmother, thank you for your unwavering belief in me. From Ranjit Singh with a peacock turban to helping me understand pump systems, thank you for everything. And most importantly, to my brother and best friend, Alex Mathew, thank you for being my goalkeeper. 


\title{
Evaluation of CFD Predictions Using Thermal Field Measurements on a Simulated Film Cooled Turbine Blade Leading Edge
}

\author{
Sibi Mathew, M.S.E \\ The University of Texas at Austin, 2010 \\ Supervisor: David G. Bogard \\ Computations and experiments were run to study adiabatic effectiveness and thermal \\ field contours for a simulated turbine blade leading edge. The RKE and SST k- $\omega$ \\ turbulence models were used for the computational simulations. Predictions of RKE \\ model for laterally averaged adiabatic effectiveness matched the experimental values. \\ The computational simulations showed different flowfield for the coolant exiting the \\ stagnation line row of holes. Both the experiments and SST k- $\omega$ simulations predicted \\ coolant separation at the stagnation plane. Also, the downstream spreading of the coolant \\ exiting the stagnation row of exit holes was better predicted by the SST k- $\omega$ model. At \\ the stagnation plane, experimental thermal field measurements showed greater diffusion \\ of the coolant into the mainstream than predicted by both turbulence models. Reasons for \\ increased diffusion were examined. Thermal field comparison downstream of the \\ offstagnation row of exit holes showed that the computational simulations and the \\ experiments had the same general shape for the offstagnation coolant jet. But the \\ computational simulations predicted greater diffusion of coolant in the direction normal \\ to the surface than seen in the experiments.
}




\section{Table of Contents}

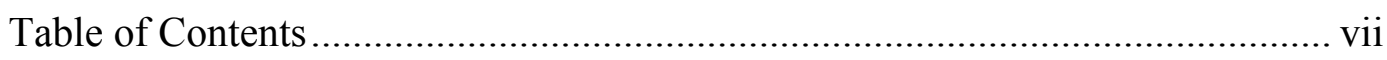

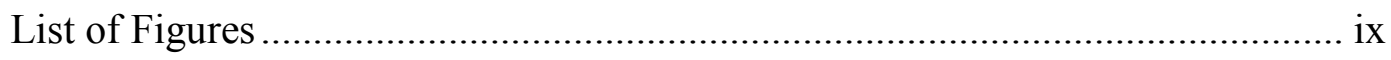

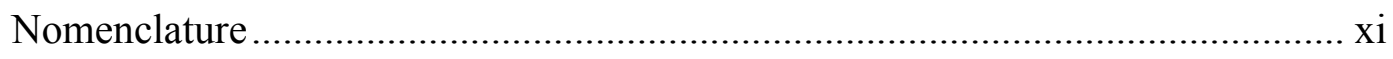

Chapter 1: Introduction and Literature review......................................................

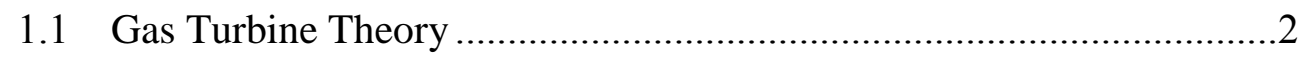

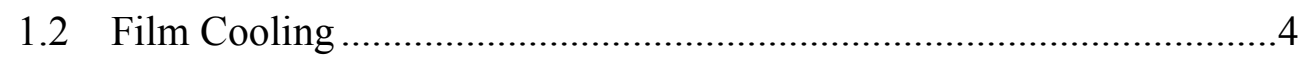

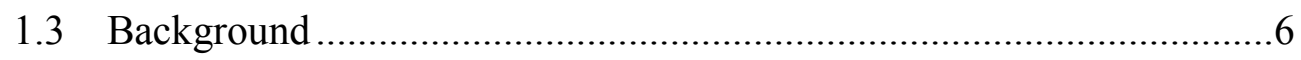

1.3.1 Turbine Airfoil Cooling .........................................................6

1.3.2 Biot Number Analysis ..........................................................10

1.3.3 Experimental Studies ..............................................................12

1.3.4 Computational Modeling ....................................................15

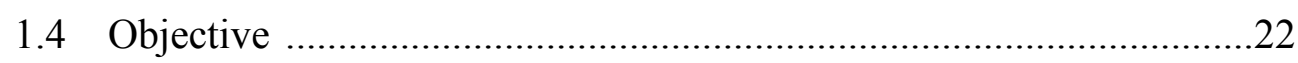

Chapter 2: Experimnetal and Computational Setup.............................................23

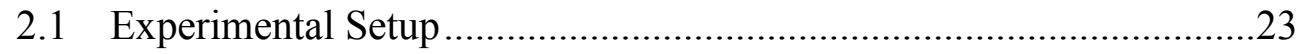

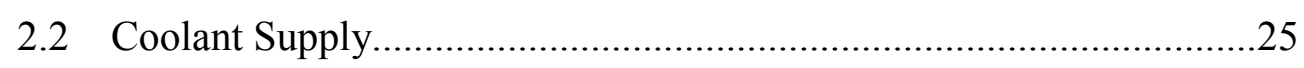

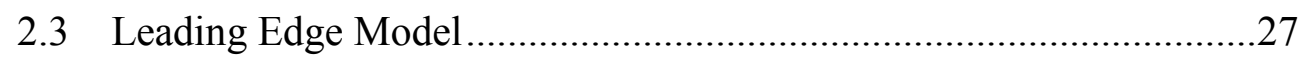

2.4 Test Section Instrumentation .............................................................

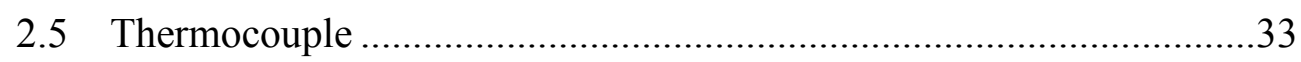

2.5.1 Thermocouple Conduction Error …………………………........33

2.5.2 Thermocouple Design .............................................................

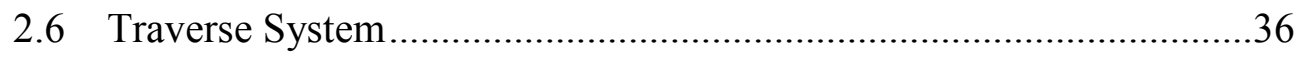

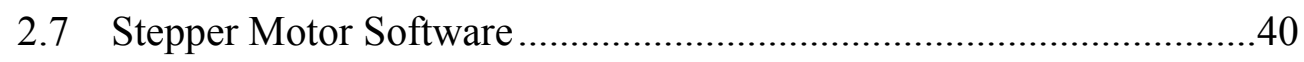

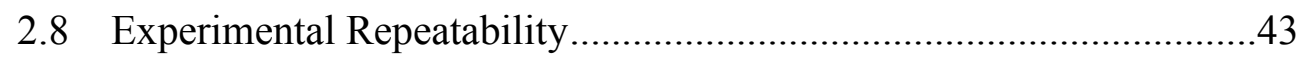

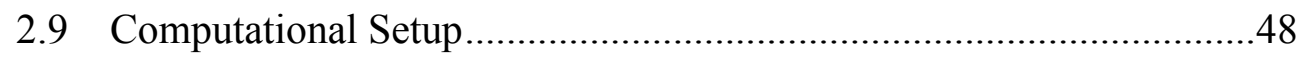

2.10 Computational Models Specification...................................................52

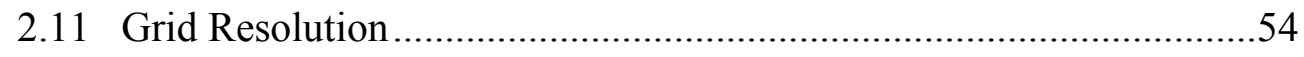

2.5 Convergence and Grid Resolution .....................................................56 


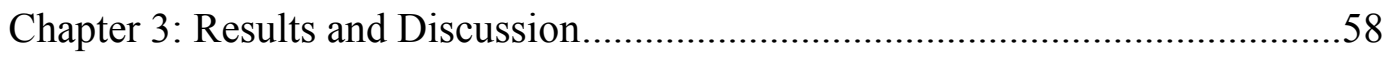

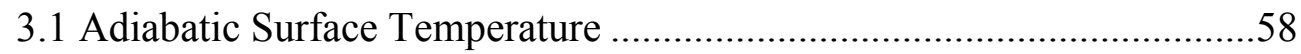

3.2 Analysis of Thermal Field in the Stagnation Plane .................................65

3.2.1 Velocity at the Coolant Exit...........................................................66

3.2.2 Separation along the Stagnation Plane.........................................69

3.2.3 Mixing of the Coolant with the Mainstream.................................72

3.2.4 Temperature Profiles in the Stagnation plane ...............................74

3.2.5 Turbulence Characteristics............................................................77

3.3 Between the Stagnation and Off Stagnation Row of Holes ......................83

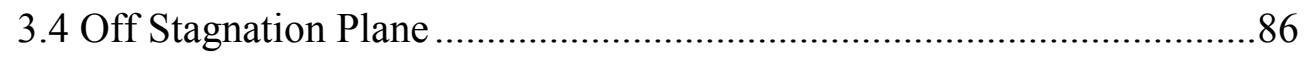

3.4.1 Thermal Field Measurements ………………………………........86

3.4.2 Temperature Profiles...............................................................90

3.5 Region of No Coolant Influence .........................................................

Chapter 4: Conclusions .................................................................................100

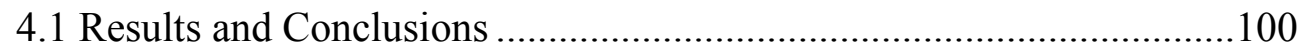

4.2 Recommendations for Future Work.......................................................103

Appendix : Low Freestream Turbulence ............................................................105

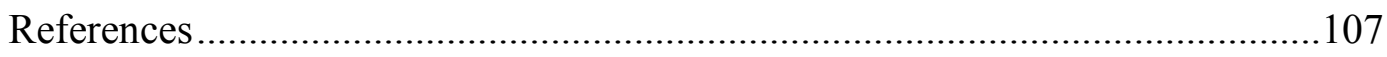

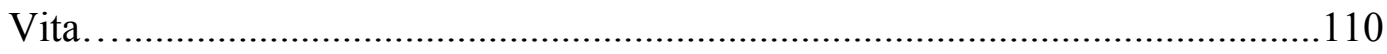




\section{List of Figures}

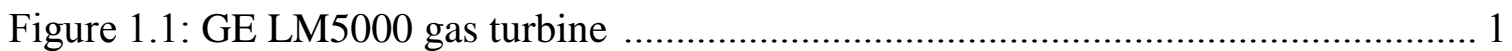

Figure 1.2: Ideal Brayton cycle for a gas turbine .......................................................... 2

Figure 1.3: Gas turbine blade with film cooling hole ............................................... 5

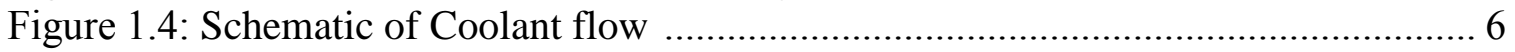

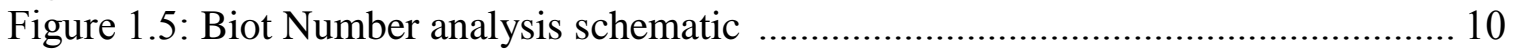

Figure 2.1: Schematic of wind tunnel test section and secondary cooling loop .............. 24

Figure 2.2: Side view of leading edge model ............................................................... 28

Figure 2.3: Front view schematic of leading edge model .......................................... 29

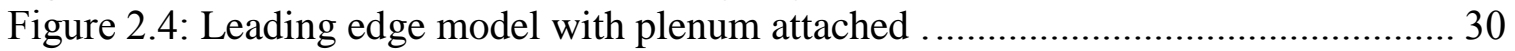

Figure 2.5: Picture of the Traverse System ................................................................. 37

Figure 2.6: Top view of Stagnation plane and schematic of the probe junction and probe

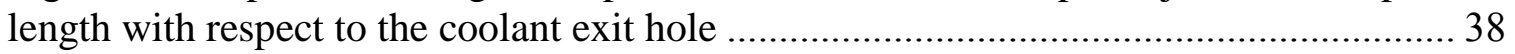

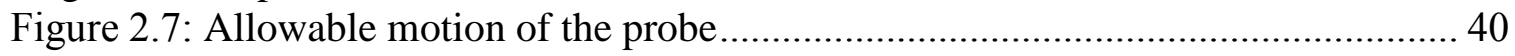

Figure 2.8: LabVIEW Signal Express Code used for stepper motor control................... 42

Figure 2.9: 'Allow Hardware reuse' control that allows switching between voltages

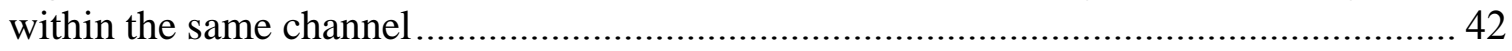

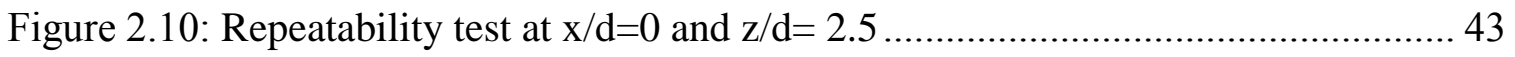

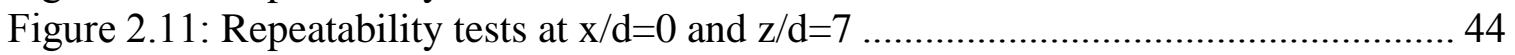

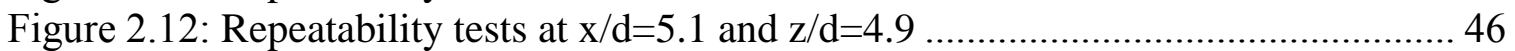

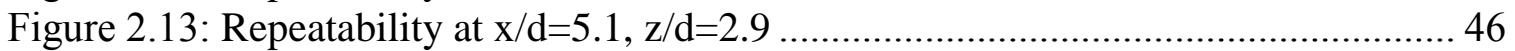

Figure 2.14: Side view of model........................................................................... 48

Figure 2.15: Comparison of Computational domains for RKE model for $\mathrm{M}=2$ and

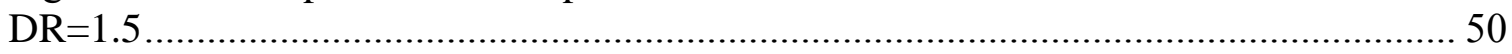

Figure 2.16: Comparison of $\phi$ along stagnation line with different computational domains

50

Figure 2.17: Schematic of leading edge domain....................................................... 52

Figure 2.18: Grid resolution for the stagnation plane- (a) k- $\omega$ and (b) k- $-\varepsilon \ldots \ldots \ldots \ldots \ldots \ldots \ldots . . . . . . . . . . .55$

Figure 3.1: Contours at $\mathrm{M}=2$ and $\mathrm{DR}=1.5-$ (a) $\mathrm{k}-\omega,(\mathrm{b}) \mathrm{k}-\varepsilon$ and (c) Experimental....... 62

Figure 3.2: Laterally Averaged $\eta$ for the leading edge model for $M=2$ and $D R=1.5 \ldots \ldots .62$

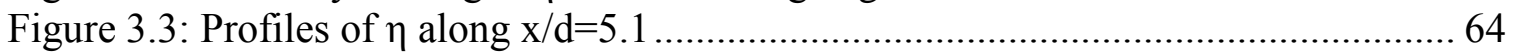

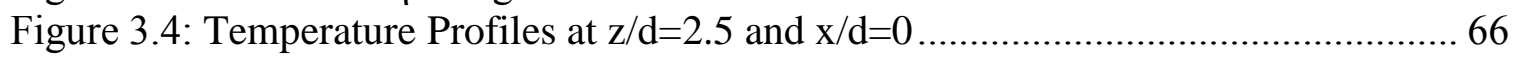

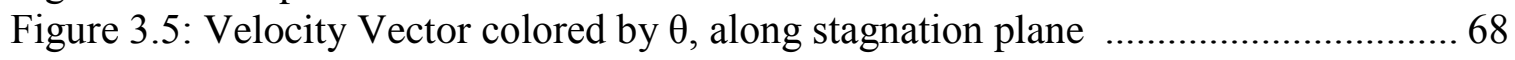

Figure 3.6: Stagnation Plane thermal profile- (a) Experimental, (b) k- $\omega$ and (c) k- $\varepsilon$..... 70

Figure 3.7: Off the wall profiles at the stagnation plane............................................. 72

Figure 3.8: Diffusion length thickness along the spanwise direction for $\mathrm{x} / \mathrm{d}=0$............ 73

Figure 3.9: Temperature Profile at $\mathrm{z} / \mathrm{d}=0$ along the stagnation plane ........................... 75

Figure 3.10: Temperature Profile at $\mathrm{z} / \mathrm{d}=2$ along the stagnation plane ........................... 76

Figure 3.11: Temperature Profile at $\mathrm{z} / \mathrm{d}=4$ along the stagnation plane ............................ 77

Figure 3.12: Contours of Turbulent Viscosity $(\mathrm{kg} / \mathrm{m}-\mathrm{s})$ at Stagnation plane ................... 79

Figure 3.13. Turbulent Viscosity Profile at $\mathrm{z} / \mathrm{d}=0$ and along stagnation plane................ 80

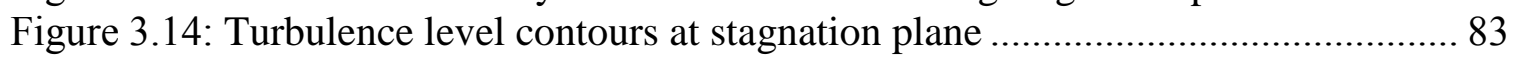

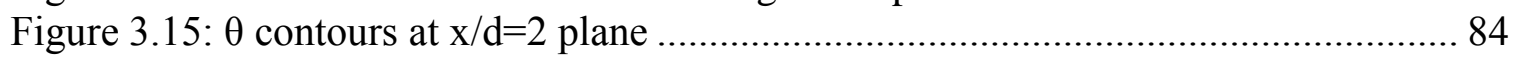




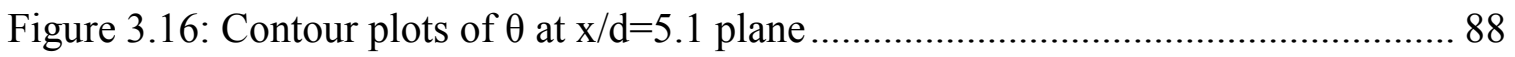

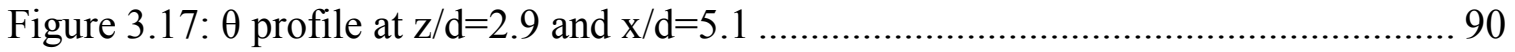

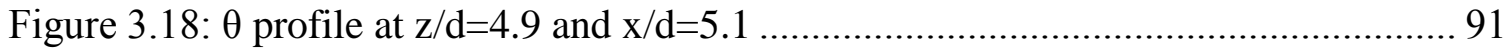

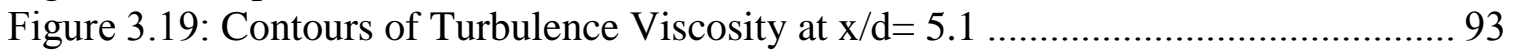

Figure 3.20: Velocity magnitude profile at location of lowest $\phi$ and $\mathrm{x} / \mathrm{d}=12.54 \ldots \ldots \ldots \ldots . .96$

Figure 3.21: Velocity magnitude profile at location of lowest $\phi$ and $x / d=12.54$, near wall

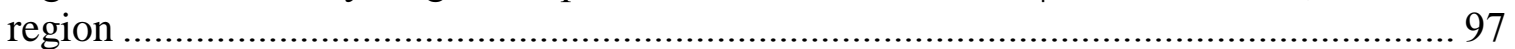

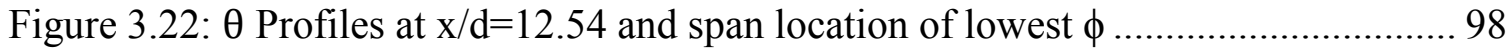

Figure 3.23: Turbulence Intensity Profiles at $\mathrm{x} / \mathrm{d}=12.54$ and span location of lowest $\phi . .99$

Figure A.1: Contours of $\theta$ along the stagnation plane with varying freestream turbulence

105

Figure A.2: Contours of overall effectiveness with varying freestream turbulence ..... 106 


\section{Nomenclature}

\begin{tabular}{|l|l|}
\hline$A$ & Area of the hole \\
\hline$B l$ & Biot Number $=h t / k$ \\
\hline$D R$ & Density ratio $=\rho_{d} \rho_{\infty}$ \\
\hline$d$ & Hole diameter \\
\hline$h_{f}$ & Heat transfer coefficient with film cooling \\
\hline$h_{0}$ & Heat transfer coefficient without film cooling \\
\hline$h_{f} / h_{0}$ & Heat transfer augmentation \\
\hline$k$ & Thermal conductivity \\
\hline$l$ & Thickness of airfoil \\
\hline$M$ & Blowing ratio $=\rho_{c} U_{d} \rho_{\infty} U_{\infty}$ \\
\hline$\dot{m}$ & Mass flow rate \\
\hline$N u$ & Nusselt number $=h l / k$ \\
\hline$p$ & Hole-to-hole pitch \\
\hline$q$ & Heat flux \\
\hline$R e$ & Reynolds number \\
\hline$T$ & Temperature \\
\hline$T u$ & Turbulence intensity level \\
\hline$u$ & Velocity \\
\hline$x$ & Streamwise coordinate \\
\hline$y+$ & Non-dimensional distance from wall $=\rho u_{\tau} y / \mu$ \\
\hline$z$ & Spanwise coordinate \\
\hline$\delta$ & Edge of the boundary layer \\
\hline$\eta$ & Adiabatic effectiveness $=\left(T_{a w}-T_{\infty}\right) /\left(T_{c}-T_{\infty}\right)$ \\
\hline$\phi$ & Overall Effectiveness $=\left(T_{w}-T_{\infty}\right) /\left(T_{c}-T_{\infty}\right)$ \\
\hline$\Lambda$ & Integral length scale \\
\hline$\mu_{T}$ & Turbulence viscosity \\
\hline$\theta$ & Normalized temperature $=\left(T_{g a s}-T_{\infty}\right) /\left(T_{c}-T_{\infty}\right)$ \\
\hline$\theta$ & Normalized temperature $=\left(T_{g a s}-T_{\infty}\right) /\left(T_{\text {coolant }, e x i t}-T_{\infty}\right)$ \\
\hline$\rho$ & Density \\
\hline & \\
\hline
\end{tabular}


$\underline{\text { Subscripts and Superscripts }}$

\begin{tabular}{|l|l|}
\hline AW & Adiabatic wall \\
\hline$c$ & Coolant \\
\hline CFD & Computational Fluid Dynamics \\
\hline cond & Conducting \\
\hline ext & Property evaluated on the external surface \\
\hline$f$ & With film cooling \\
\hline in & At the entry of the hole \\
\hline int & Property evaluated on the internal surface \\
\hline junc & Junction \\
\hline off-stag & Off Stagnation \\
\hline out & At the exit of the hole \\
\hline pred & Predicted \\
\hline stag & Stagnation \\
\hline w & Property evaluated at the wall \\
\hline o & No film cooling injection \\
\hline$\infty$ & Mainstream \\
\hline- & Lateral average \\
\hline
\end{tabular}




\section{Chapter 1: Introduction and Literature Review}

Gas turbine engines are vital power generators in the modern world. They are used in the transportation sector to power planes, helicopters and ships. Turbines are also used extensively in power plants due to their ease of startup, high efficiency and low emissions. Gas turbines operate on a Brayton cycle and consists of four basic stages- a compressor where the inlet cold air is compressed, a combustor where the fuel is combusted with air, a turbine stage where power is extracted and an exhaust nozzle, as shown in Figure 1.1.

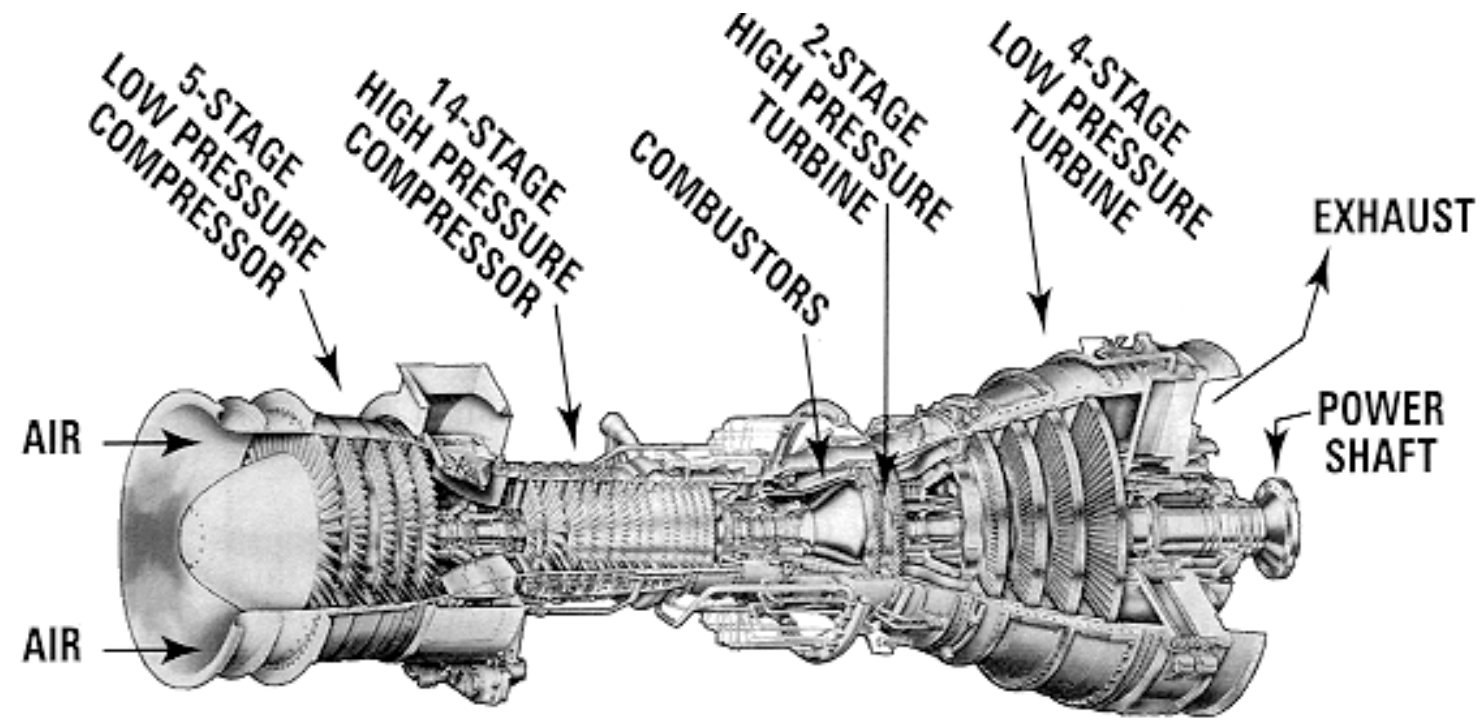

Figure 1.1- GE LM5000 gas turbine

http://www.dieselduck.ca/machine/01\%20prime\%20movers/gas_turbine/gas_turbine.htm

With higher power demands, the efficiencies of gas turbines are constantly pushed upward. Industrial grade gas turbines can achieve efficiencies close to $40 \%$ with aero derivative turbines having higher efficiencies. The first stage of the turbine section of a 
gas turbines usually experiences high thermal and inertial loads and are thus more subject to failure. Thus improving the life span of these high pressure turbines is critical to the lifespan of the engine.

\subsection{Gas turbine Theory}

Most gas turbines operate on a cycle similar to the Brayton cycle, shown in Figure 1.2. The first step of the ideal Brayton cycle consists of adiabatic compression of air that enters the engine. Then heat is added to the compressed air in the combustor and the process is ideally at constant pressure. Upon leaving the combustor stage, the high pressure hot gas is allowed to expand within the diffusive turbine stage. Ideally, this part of the cycle is adiabatic and mechanical work is extracted by the rotating of the gas turbine blades. Part of the power extracted within the turbine stage is used to power the compressor stage of the engine with the rest used for auxiliary applications. Finally the diffused gas is allowed to exit the engine.
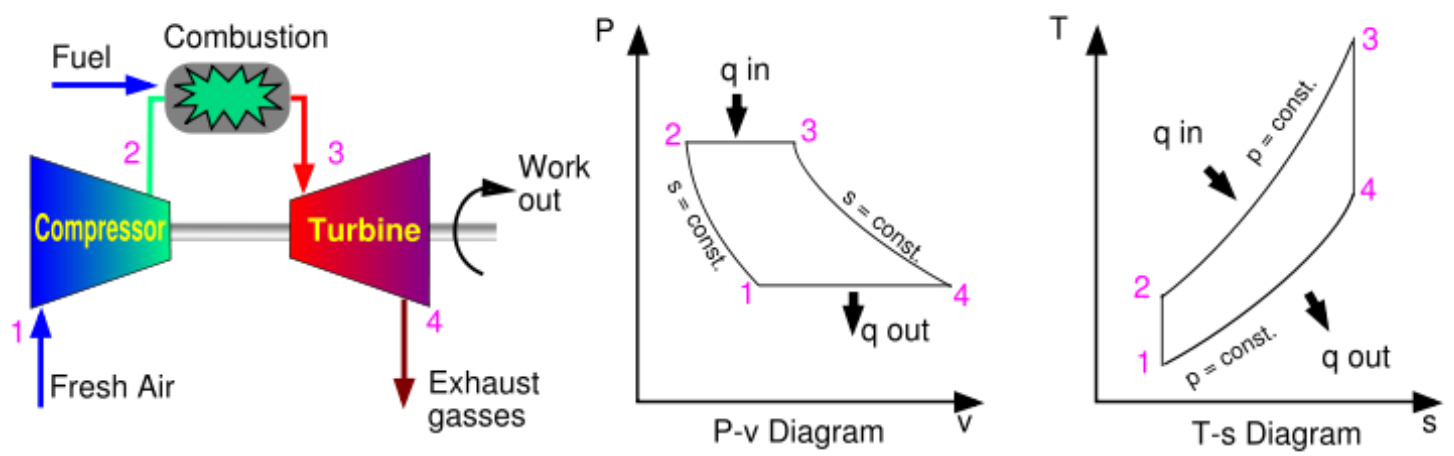

Idealized Brayton Cycle

Figure 1.2 - Ideal Brayton cycle for a gas turbine http://www.braytonenergy.net 
The efficiency of the engine is defined as

$$
\eta=\frac{\text { Net } \text { work output }}{\text { Net work input }}
$$

For an ideal process, the net work output by a gas turbine engine is $\left(h_{3}-h_{4}\right)-\left(h_{2}-h_{1}\right)$, i.e the work extracted by the turbine minus the work need to compress the gas. The net work input is the heat added by burning fuel and can be defined as $\left(\mathrm{h}_{3}-\mathrm{h}_{2}\right)$. Assuming constant heat capacity, the efficiency can be redefined as

$$
\eta=\frac{\left(T_{3}-T_{4}\right)-\left(T_{2}-T_{1}\right)}{\left(T_{3}-T_{2}\right)}
$$

Rearranging

$$
\eta=1-\frac{\left(T_{4}-T_{1}\right)}{\left(T_{3}-T_{2}\right)}
$$

Thus it can be seen that the engine efficiency can be direct related to the temperature of the hot gases exiting the combustor $\left(\mathrm{T}_{3}\right)$ and subsequently entering the turbine stage. Higher turbine inlet temperatures translate to higher efficiencies for the engine. The thrust output of an aero gas turbine also increases with higher temperatures. Most modern turbines operate with turbine inlet temperatures of $1700^{\circ} \mathrm{C}$ with the maximum temperature being dictated by the fatigue temperature of the turbine material. With advances in alloy and manufacturing technology, turbine operators have been able to increase the engine efficiency by having higher turbine inlet temperatures. Even then, other cooling techniques become necessary for maintaining turbine blade integrity. Film 
cooling is one of these techniques that is widely used and is the topic of study of this thesis.

\subsection{Film Cooling}

Film cooling consists of bleeding a relatively small amount of air from the compressor stage of the engine. Between $20 \%$ and $25 \%$ of the total volume of air within the compressor is used in the cooling process. With such a high volume of bled air, the efficiency of the gas turbine can be affected. Film cooling schemes thus try to minimize the amount of air used for cooling. The bled off air is routed around the hot combustor region and is colder than the hot mainstream gases that impact the turbine blades. This colder air is referred to as coolant and is the basis of film cooling. Film cooling is a significant attribute in the initial stages of the turbine section. The blades and vanes within this section are closest to the hot combustor and experience the impact of high pressure, hot mainstream gases. Figure 1.3 show a gas turbine blade with film cooling holes. The leading edge region of these high pressure turbine blades (HPT) experiences the most thermal load for the blade. The stagnation region has thin thermal boundary layer and high convective heat transfer coefficients and is thus highly dependent on film

cooling. The computational and experimental models used in this thesis focused on the leading edge region. 


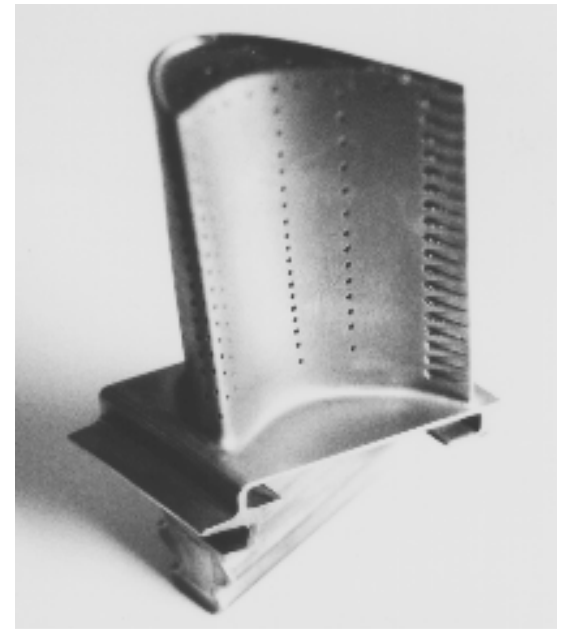

Figure 1.3- Gas turbine blade with film cooling hole http://www.me.umn.edu/labs/tcht/measurements/what.html

The colder air bled off from the compressor is initially routed through serpentine channels within the blade structure. Rib tabulators and impingement plates are also employed where the coolant flow is directed in the form of high velocity jets onto the internal surface of the blade leading edge. The impingement of the jets causes significant increases in convective cooling within the blade interior structure.

The coolant is also allowed to exit the blade through small coolant hole located on the external surface. The exiting coolant forms a cold protective layer of gas above the blade surface and reduces the transfer of heat from the hot mainstream gases to the metal surface. The thickness and effectiveness of the protective coolant layer is dependent on the several factors such as the momentum of the exiting coolant, its density, location and shape of the coolant exit holes among others. 


\subsection{Background}

\subsubsection{Turbine Airfoil cooling}

Film cooling involves significant and complex interactions between two turbulent flow fields. This requires the creation of several non dimensional parameters that can help quantify the efficiency of the cooling process. The schematic in Figure 1.4 shows the flow of coolant.

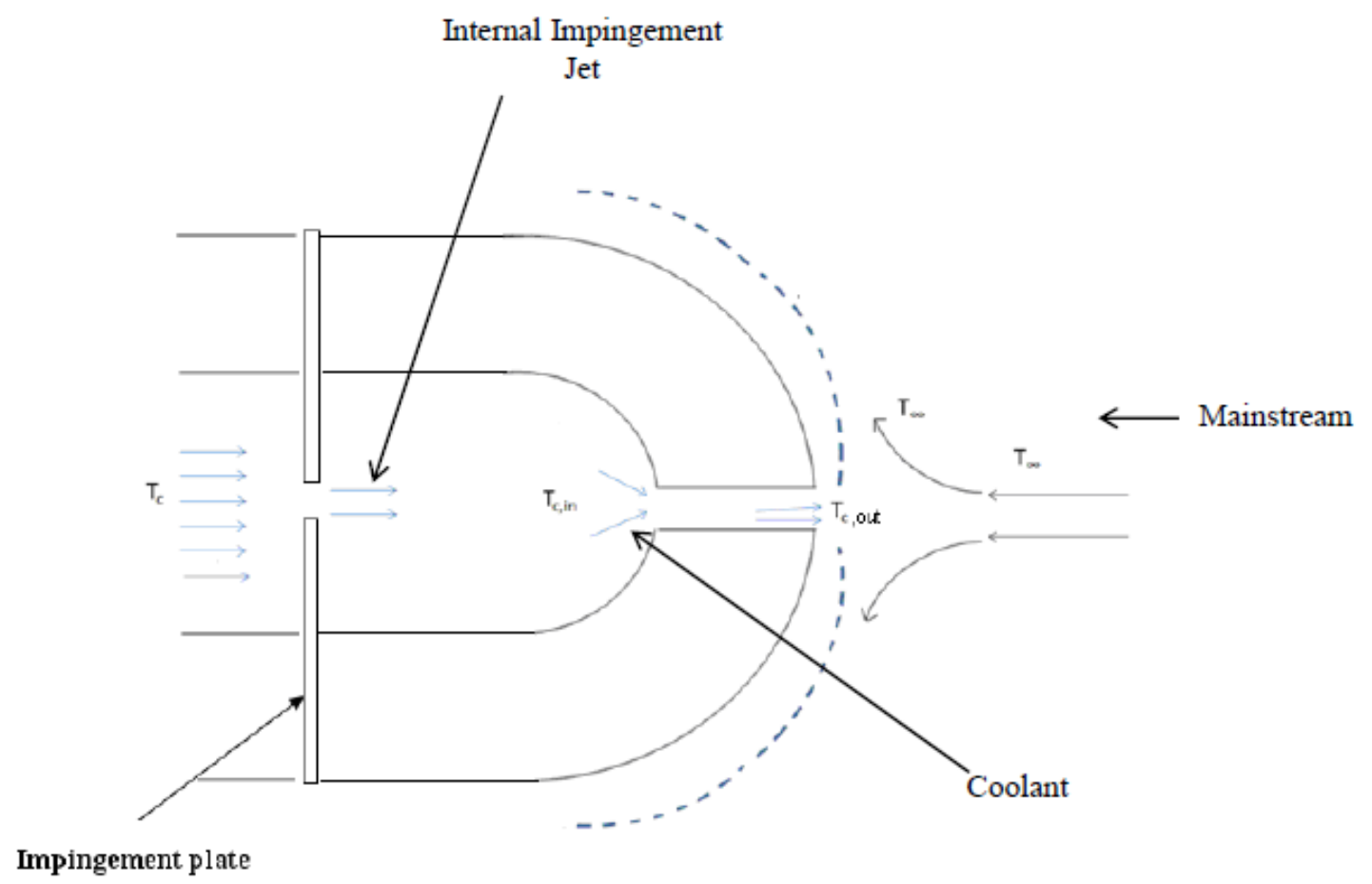

Figure 1.4- Schematic of Coolant flow

The temperature of the coolant exiting the impingement plate hole is defined as $T_{c}$ and the hot mainstream gas is defined as temperature $\mathrm{T}_{\infty}$. The impingement of the jet onto the model inner wall results in heat transfer from the wall to the coolant and thus results in an increase in coolant temperature from $T_{c}$ to $T_{c, \text { in }}$. The high speed flow of coolant 
through the cooling holes also results in an increase in coolant temperature with the coolant exit temperature defined as $\mathrm{T}_{\mathrm{c}, \text { out }}$. The exiting coolant then proceeds to form a protective coolant layer over the blade surface resulting in the lowering of the blade surface temperature. It must be noted also that while the development of the colder gas layer over the surface results in a lower metal temperatures, the jet interaction with the mainstream also results in an increase in the convective heat transfer coefficient. Johnston (1999) analyzed laterally averaged heat transfer augmentation $\left(\mathrm{h}_{\mathrm{f}} / \mathrm{h}_{\mathrm{o}}\right)$ for a leading edge model at $M=2$, where $h_{f}$ is heat transfer coefficient with film cooling and $h_{o}$ is heat transfer coefficient without film cooling. The augmentation was greater than 1 throughout the length of the model and thus showed increase in heat transfer coefficient with film cooling.

For better comparison of different film cooling schemes, the metal surface temperature is usually defined in terms of non dimensional temperature. For an adiabatic model, the non dimensional surface temperature is defined as adiabatic effectiveness $(\eta)$. If $T_{A W}$ is the surface temperature of the adiabatic model, then

$$
\eta=\frac{T_{\infty}-T_{A W}}{T_{\infty}-T_{C}}
$$

For an adiabatic model, the surface temperature is equal to the temperature of the gas flowing over the model at that location because the model is unaffected by conduction. The adiabatic effectiveness can also be seen a measure of the amount of mixing of the coolant with the mainstream gas at that location above the model. A $\eta$ value of 1 
indicates gas temperatures equal to the coolant exit temperatures while 0 indicates the absence of film cooling.

For a conducting model, the surface temperature is expressed as a non dimensionalized parameter called overall effectiveness $(\phi)$. Overall effectiveness of the model can be directly compared to $\phi$ values in real engine applications if scaled properly. With $T_{w}$ indicating surface temperature of the conducting model, $\phi$ is defined as

$$
\phi=\frac{T_{\infty}-T_{w}}{T_{\infty}-T_{c}}
$$

A conducting model experiences both conduction effects through the model interior and also convective heat transfer due to gas flow above the surface. The driving temperature for convective heat transfer is the temperature of the gas directly above the model at that location. Since $T_{A W}$ is a measure of the gas temperature above the model, it can also be viewed as the driving temperature for heat transfer into a conducting model. Harrison and Bogard (2008) studied the usage of $T_{A W}$ as the driving temperature by simulating film cooling on conducting and adiabatic flat plates. The results showed that this assumption holds for most regions of the flat plate except in locations very close to the coolant exit hole.

As the coolant jet flows farther away from the exiting hole, the coolant temperatures increase further due to mixing with the hot mainstream gases. With the local gas temperature defined as $T_{\text {gas }}$, a non dimensional gas temperature $\theta$ is defined as 


$$
\theta=\frac{T_{\infty}-T_{g a s}}{T_{\infty}-T_{c}}
$$

A unit value of $\theta$ means a local gas temperature equal to the coolant exit temperature while a $\theta$ value of zero corresponds to a gas temperature equal to the mainstream temperature.

Blowing ratio and density ratio are other non dimensional parameters that are used to define the flow of the coolant. The ratio of coolant jet to mainstream mass flux is defined as blowing ratio $(\mathrm{M})$

$$
M=\frac{\rho_{C} U_{C}}{\rho_{\infty} U_{\infty}}
$$

Where $\rho_{C}$ and $\rho_{\infty}$ are coolant exit density and mainstream density, respectively, and $U_{C}$ and $U_{\infty}$ defined as coolant average exit velocity and free stream velocity, respectively.

Using similar variables, the density ratio is the ratio of coolant to free stream densities

$$
D R=\frac{\rho_{C}}{\rho_{\infty}}
$$




\subsubsection{Biot Number Analysis}

With the experimental and the real engine models having different dimensions, certain parameters have to be scaled for effective comparison between the two. The Reynolds number can be matched between the experimental and real models. With Nusselt number being a function of Reynolds number and Prandtl number and the Prandtl number not varying much in the engine temperature range, the experimental and real models will also have matched Nusselt number. A schematic of the energy balance through a conducting model is shown in Figure 1.5.

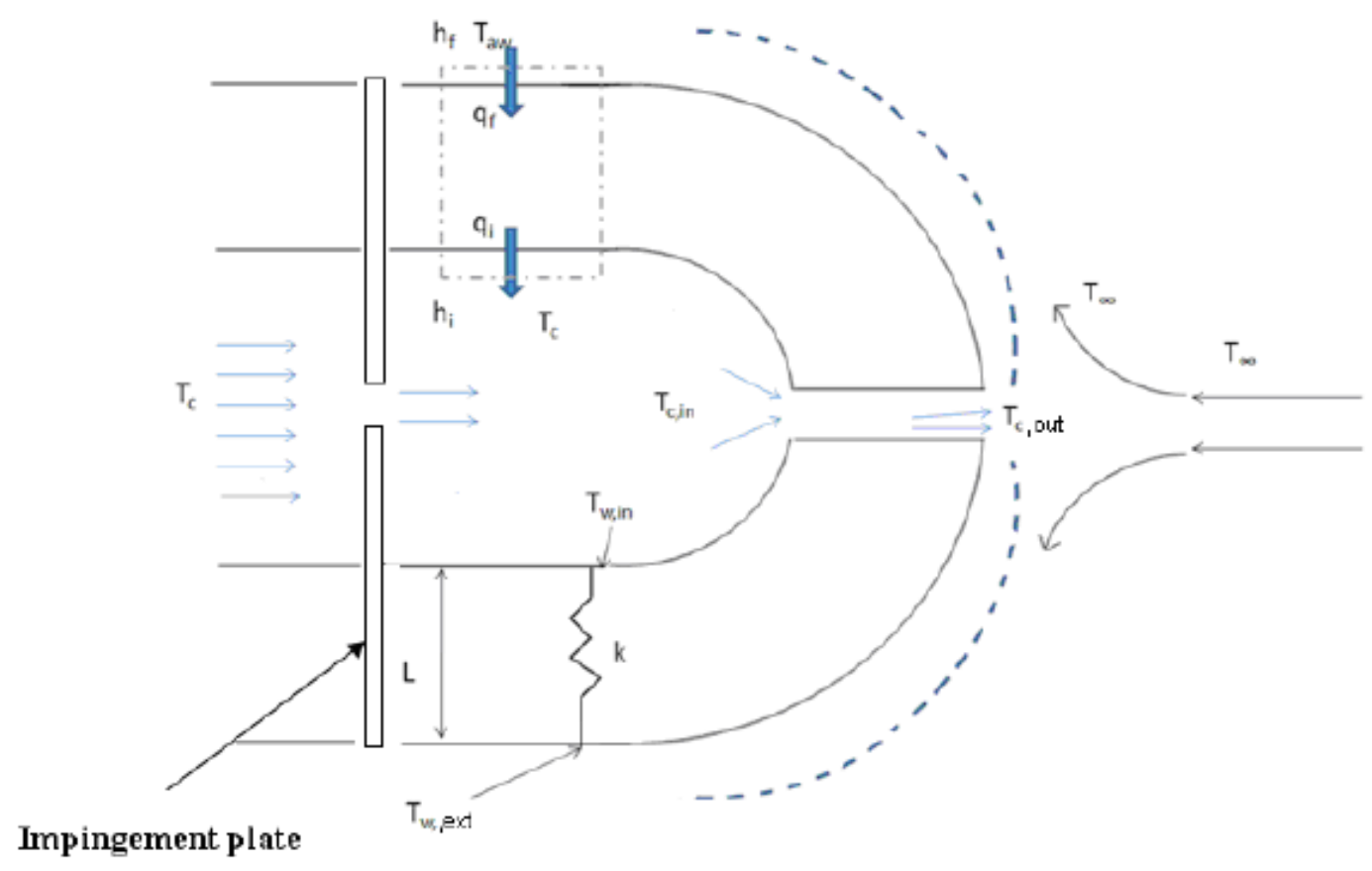

Figure 1.5 - Biot Number analysis schematic

For conducting models, other parameters have to be matched in order for effective comparison of the non dimensional external surface temperature (overall effectiveness). 
Albert et al. (2004) demonstrated with a 1-D analysis that matching the Biot number along with the Reynolds number and the flow geometry is sufficient for accurate comparison of the overall effectiveness.

The heat flux occurring at the external surface is defined as;

$$
q_{f}=h_{f}\left(T_{A W}-T_{w, e x t}\right)
$$

The internal heat flux from the solid to the coolant within the model is described as;

$$
q_{\text {int }}=h_{\text {int }}\left(T_{w, i n t}-T_{c}\right)
$$

And the 1-D conduction through the model is described as;

$$
q_{\text {cond }}=\frac{k_{\text {model }}\left(T_{w, e x t}-T_{w, i n t}\right)}{l}
$$

Where $k_{\text {model }}$ is the thermal conductivity of the model and $l$ is model thickness.

One dimensional energy conservation across the model reveals that

$$
\begin{gathered}
q_{f}=q_{\text {int }}=q_{\text {cond }} \\
\text { i.e } \quad h_{f}\left(T_{A W}-T_{w, \text { ext }}\right)=h_{\text {int }}\left(T_{w, \text { int }}-T_{c}\right)=\frac{k_{\text {model }}\left(T_{w, \text { ext }}-T_{w, \text { int }}\right)}{l}
\end{gathered}
$$

Rearranging, it can be shown that 


$$
\frac{T_{A W}-T_{w, e x t}}{T_{A W}-T_{c}}=\frac{1}{1+\frac{h_{f}}{h_{\text {int }}}+B i}
$$

Where $B i=\frac{h_{f} l}{k}$, a ratio of the convective heat transfer for the model to the conductive heat transfer within the model. The heat transfer coefficient ratio is dependent on the Reynolds number and Prandtl number of the external and internal flows . By matching the flow conditions, the heat transfer coefficient ratios can be assumed to be similar to the engine. Thus for a conducting model, matching the Biot number gives valid overall effectiveness comparison.

\subsubsection{Experimental Studies}

Thermal field studies of coolant flow can be used to study the coolant flowfield and coolant dispersion into the mainstream. Researchers have studied thermal field measurements for different flow regimes over both flat plates and section of the leading edge.

Coulthard et al. (2007) studied film cooling on an adiabatic flat plate model using an open loop wind tunnel, with the test section exposed to the still air in the room. Cooling hole geometry consisted of a single row of holes oriented parallel to the stream wise direction. The thermal field data was collected using cold wire anemometry. Boundary layer probes with $1.27 \mu \mathrm{m}$ diameter platinum sensors were used. For steady coolant flow and $M>0.5$, the thermal profiles shows regions of coldest temperatures further away from the model surface and hence shows separation of the coolant. Lateral average $\eta$ values 
were consistently higher for the lower blowing ratios $(\mathrm{M}<0.5)$ especially near the exit hole.

Teng et al. (2001) studied thermal field measurements on the suction side of a adiabatic turbine blade. This study focused on film cooling performance on curved surface with strong flow acceleration conditions. The thermal field measurements were taken using a traversing fine gauge thermocouple probe. The thermocouple bead size is $0.01 \mathrm{~cm}$ and the probe size was $0.0254 \mathrm{~cm}$. For cylindrical holes and steady flow condition, the thermal fields at $\mathrm{x} / \mathrm{d}=5$ and $\mathrm{x} / \mathrm{d}=10$ downstream locations showed that the core of the coolant jet lifts off from the surface at $M=1.2$. Thermal profiles show the film coverage area was narrow in the spanwise direction but longer in the boundary layer direction with coolant extending up to $\mathrm{y} / \mathrm{d}=2$ from the surface.

Cutbirth and Bogard (2002) measured thermal and velocity field measurements in the showerhead region of an adiabatic turbine vane. The showerhead region consists of 6 rows of staggered holes with the stagnation line located at the center of the third row from the pressure side. The thermal and velocity field measurements were conducted at $\mathrm{DR}=1.2$. Flow visualization data revealed that coolant separation occurs at the stagnation plane even for low $\mathrm{M}_{\mathrm{sh}}=0.5$. For $\mathrm{M}_{\mathrm{sh}}=1.5$, thermal field measurements showed that the core of the coolant jet was located at $\mathrm{y} / \mathrm{d}=2$ from the surface at the stagnation plane and the coolant mixing layer extends to $y / d=4.5$ from the surface. The surface effective plots also revealed that $\mathrm{M}_{\mathrm{sh}}=1.5$ resulted in large increase in $\eta$ levels in the showerhead region. At this high blowing ratio, the mass flow of coolant was enough for jet to jet interaction 
and hence provided coolant coverage throughout the span length. This prevented penetration of the mainstream towards the model surface. This paper provided thermal field measurements for a plane at one span location and thus does not show the growth of the coolant diffusion layer along the span length, between cooling holes.

Cruse et al. (1997) studied film cooling on a leading edge adiabatic model with cooling rows located at the stagnation line and another row of holes located 3.5d downstream of stagnation line. This geometry is similar to the leading edge model studied in this thesis. Along with adiabatic effectiveness $(\eta)$, the study also measured thermal field contours at several downstream locations. The thermal field data was measured using thermocouple rake that included 10 type E thermocouple sensors. Each sensor had a diameter of $0.1 \mathrm{~mm}$ and has a spacing of $1 \mathrm{~mm}$ between sensors. At $\mathrm{DR}=1.8, \mathrm{M}=2$ and low freestream turbulence of $0.5 \%$, thermal field data at $\mathrm{x} / \mathrm{d}=1.2$, i.e immediately downstream of the stagnation line row of holes, showed highest $\theta$ values of 0.3 and the coolant was distributed across the one pitch length. Thermal field data at $\mathrm{x} / \mathrm{d}=4.9$ revealed that interaction with the mainstream resulted in the outer part of the offstagnation coolant being pushed away from the bulk of the coolant jet closer to the wall. The core of the offstagnation coolant jet had $\theta$ values of 0.6 and the 0.2 isotherm extended to $y / d=2$. Moving farther downstream to $\mathrm{x} / \mathrm{d}=10$, the coolant jet was dispersed considerably in the normal and lateral direction.

The leading edge model with matched Biot number, described in section 1.3.2, has been used in several studies to compare film cooling performance on an experimental 
model to a real engine. Albert et al. (2004) analyzed adiabatic and overall effectiveness for a leading edge model with forward diffused shaped holes. A Biot number of 1.2 was used for the conducting model of thermal conductivity of $1.2 \mathrm{~W} / \mathrm{m} . \mathrm{K}$. Results showed that internal cooling, on the inside surface and through the cooling holes, provide significant contribution to overall effectiveness. Mouzan et al. (2005) also used a matched Biot number leading edge model to study the validity of using net heat flux reduction as a means to predicting overall effectiveness. Maikell (2008) also measured overall effectiveness for a conducting film cooled leading edge of geometry similar to the model used in this thesis.

\subsubsection{Computational Modeling}

Computational modeling of fluid flow has become a significant aspect of engineering design. Certain CFD models solve the Navier Stokes equation (NS) using time dependant techniques. The velocity field is solved for at every point in the domain. Examples of such CFD models are Large Eddy Simulation (LES), Detached Eddy Simulations (DES) and Direct Numerical Simulations (DNS). These models account for anisotropy and unsteady effects within the flow field but require large computational grids and time for converged solutions.

Another class of CFD model is the Reynolds Averaged Navier Stokes models (RANS). These models use transport equations to model the transport of momentum and energy using averaged quantities such as mean velocity and stress. The averaged Navier Stokes equation is not closed and quantities such as Reynolds shear stress need to be 
modeled. $\mathrm{k}-\varepsilon, \mathrm{k}-\omega$, RSM are examples of turbulence models used in RANS simulation of film cooling applications. The k- $\varepsilon$ and $k-\omega$ models do not account for unsteady effects within the flow and are isotropic in nature. There is also Unsteady RANS modeling in which time dependent flow is solved where the unsteadiness is externally imposed such as a time dependent boundary conditions. This thesis describes a study in which modified versions of k- $\varepsilon$ and k- $\omega$ models called the Realizable k- $\varepsilon$ (RKE) and Shear Stress Transport (SST) k- $\omega$, respectively were used to simulate a leading edge model

The RKE model was used in this thesis because the standard k- $\varepsilon$ (SKE) had been previously found lacking in predicting jet spread rate and vorticity effects for external flows. With the development of the realizable model by Shih et al. (1995), a new formulation was employed for turbulent viscosity. The RKE model requires positivity of the Reynolds normal stresses and satisfaction of the Schwarz inequality for shear stresses. The Realizable model also accounts for vorticity in the dissipation equation and hence gives better predictions of external flows and jet spread rates compared to the standard k$\varepsilon$ model. York and Leylek (2000) indicated that adiabatic effectiveness, flow field and heat transfer coefficients were better predicted with the realizable model compared to the standard k- $\varepsilon$ model for film cooling applications on a leading edge.

The deficiency of the standard k- $\varepsilon$ model was also shown by Medic and Durbin (2002) who modified the standard k- $\varepsilon$ model by putting a limit on the turbulence time scale. Such a model limited the magnitude of turbulent kinetic energy (k). Film cooling predictions were studied on the suction and pressure side of a turbine blade model. 
Results showed the SKE predicted higher turbulence viscosity levels which resulted in greater diffusion and caused high temperatures to penetrate from the freestream closer to the blade surface. Turbulence intensity contours also showed the SKE model having significantly higher levels while the $\mathrm{T}$ bound $\mathrm{k}-\varepsilon$ and $\mathrm{v}^{2}$-f models were consistently lower.

The current thesis also employed the SST based k- $\omega$ model for film cooling predictions. The Shear Stress Transport model (SST) combines the capability of the standard k- $\omega$ to better resolve near wall flow with the capability of k- $\varepsilon$ model to better predict freestream and bulk flow aspects. A blending function is applied to provide a transition between the standard $\mathrm{k}-\omega$ and the modified $\mathrm{k}-\varepsilon$. The blending function is devised so that $\mathrm{k}-\omega$ is used in the inner half of the boundary layer with a gradual blending of $k-\omega$ and $k-\varepsilon$ through the outer half of the boundary layer. And finally the $k-\varepsilon$ is used outside the boundary layer. The SST model is better applicable in adverse pressure gradient flows and is hence a better predictor of flow separation.

Harrison and Bogard (2008) simulated film cooling on a flat plate model using the realizable k- $\varepsilon$ model (RKE), standard k- $\omega$ (SKW) and RSM turbulence models. The RSM model accounts for anisotropic variations in flow. Cylindrical holes and $\mathrm{p} / \mathrm{d}=3$ were used and the holes were oriented in the streamwise direction. The SKW model showed the best comparison with the experimental data for laterally averaged $\eta$ values downstream of the exit hole at both $M=0.5$ and $M=1$. The $R K E$ value showed worst comparison at both blowing ratios. Meanwhile, the centerline adiabatic $\eta$ was 
overpredicted by all three turbulence models with the SKW model showed worst and the RKE model showed best prediction at both blowing ratios. But the SKW and RSM curves for centerline effectiveness showed a dip near the injection hole. This behavior suggests separation and reattachment of the coolant jet along the centerline. Thus, while the SKW model predicted colder coolant temperatures, the flow physics is better predicted by this model. Harrison and Bogard (2008) also studied heat transfer coefficient on flat plate. All three models showed good agreement with heat transfer coefficient correlations for the no film cooling case $\left(\mathrm{h}_{\mathrm{o}}\right)$. For the film cooled flat plate, all three model agreed well with experimental data for laterally averaged heat transfer coefficient augmentation $\left(h_{f} / h_{o}\right)$. But along the centerline, all three models under predicted the $h_{f} / h_{o}$ values with the SKW model showing the largest under prediction far from the hole and the largest over prediction near the hole. At $M=1$, all three models showed more lateral variation of $h_{f} / h_{o}$ at $x / d=6$ than the experimental values. But, while the RKE and the RSM models showed similar lateral trends, the SKW showed a different trend from the experiments.

Laskowski et al. (2007) studied different turbulence models on film and internally cooled 1988 C3X vane using CFX v11.0 software. The standard k- $\varepsilon$ (SKE), standard k- $\omega$ (SKW), Shear Stress Transport (SST) and Omega Reynolds Stress models were used. All models except the SKE were also run with wall integration approach. Comparison of midspan temperatures showed that the wall integration approach compared better with experimental data. The SST model agreed best with the experimental data for the midspan temperature along the axial length for the internally cooled model .But all 
turbulence models showed similar levels of agreement with the midspan experimental temperature data for the film cooled model. It should be noted that while the film cooled simulation used wall integration function, the leading edge section of the vane consisted of a total of $6.4 \times 10^{6}$ cells for geometry that included 48 cooling holes. Such a mesh distribution could result in the grid not being fine enough to capture the flow field adequately and show differences between turbulence models. This thesis used a RKE grid with 4.5 million cells for a symmetric half of a leading edge that consisted of one offstagnation hole and half a stagnation hole. The SST model used in this thesis had 10 million cells for the same geometry as the RKE simulation.

Keimasi et al. (2001) studied computational modeling of jets in cross flow using the standard k- $\varepsilon$ model with wall functions and the SST k- $\omega$ model. A 3-D flowfield of square jets were injected perpendicularly into the cross flow over a flat plate. For a velocity ratio $(\mathrm{R})$ of $0.5,1$ and 1.5 , both models predict a region of reversed flow downstream of the jet exit. The models also predicted counter rotating vortex pairs at $\mathrm{x} / \mathrm{d}>1$ downstream distances. The mean velocity profiles agreed with experimental data for both models. The study also indicated that Turbulent kinetic energy $(\mathrm{k})$ values were overpredicted by both models in comparison to the experimental $\mathrm{k}$ data, especially at $\mathrm{x} / \mathrm{d}=0$ and $\mathrm{R}=0.5$.For $\mathrm{y} / \mathrm{d}>0.5$ and $\mathrm{x} / \mathrm{d}=0$, the computational $\sqrt{k} / V_{\text {jet }}$ values were twice the experimental data. The SST k- $\omega$ compared better with the experimental data than the $\mathrm{k}-\varepsilon$ for $\sqrt{k} / V_{j e t}$ for $\mathrm{y} / \mathrm{d}>1$. The authors attributed anisotropy of the flowfield as a possible source of error. The computational models considered are isotropic and hence do not account for anisotropy of the complex flowfield. The grid used in the study consisted of 
265,000 grid points for computational domain of 45D $\times 20 \mathrm{D} \times 3 \mathrm{D}$ and it is noted that the study used the same grid for both turbulence models. Meanwhile, the CFD simulations studied in this thesis used a more refined grid and the two turbulence models used different grids.

There have been a number of studies that validated CFD codes using surface contours of adiabatic effectiveness and overall effectiveness. York and Leylek (2002) compared their CFD results to experimental adiabatic effectiveness plots but did not compare thermal field data. Laskowski et al. (2007) validated their full vane simulation using only experimental temperature and heat transfer coefficient data along the model centerline. While analysis of the surface contours gives a footprint of the coolant as its travels over the models, it does not describe coolant diffusion into the mainstream. Also, it does not give a good idea of the coolant flowfield above the model surface such as the extent of coolant separation or entrainment of the mainstream by the offstagnation jet. Thermal field analyses better help validate CFD codes in regard to coolant flow fields as it helps quantify coolant diffusion, separation and other flowfield effects.

Lin and Shih (2001) compared thermal field measurements from the SST turbulence model to experimental data by Cruse et al. (1997). A near adiabatic leading edge model with stagnation and offstagnation rows of cylindrical holes were used in the study. The study used the CFL3D code based on the cell centered finite volume method. The grid used had at least 5 grid nodes within a $\mathrm{y}^{+}$of 5 and the grid spacing next to the walls was kept constant for at least three grid points to $\mathrm{v}$ the turbulent boundary layer. The domain 
had a total of 1.78 million grid points. At $\mathrm{x} / \mathrm{d}=1.24$, thermal field comparisons showed the lateral and normal spreading of the coolant was comparable for SST and the experiments but the core of the CFD coolant jet was significantly colder than the experimental values. The highest CFD $\theta$ value was 0.8 compared to the highest experimental $\theta$ of 0.3 .Downstream of the offstagnation hole, at $\mathrm{x} / \mathrm{d}=4.86$, the lateral spreading of the offstagnation jet above the model $(\mathrm{z} / \mathrm{d}=3.5)$ was accurately predicted. But again, the core of the CFD jet was colder and had highest $\theta$ value of 0.9 compared to the experimental data which showed highest $\theta$ of 0.6 Both CFD and experiments showed stagnation coolant presence at similar $\mathrm{z} / \mathrm{d}$ locations for the $\mathrm{x} / \mathrm{d}=4.98$ plane . Also, analysis of the CFD flowfield at the stagnation plane revealed separation of the stagnation coolant and with a p/d=7.6, the stagnation plane also showed interaction between coolant jets within the same row. 


\subsection{Objective}

The objective of this thesis was to validate the RKE and SST k- $\omega$ CFD models with experimental data for a film cooled leading edge model. The RKE and SST model were chosen because of their superior performance in predicting film cooling results when compared to other RANS turbulence models. The CFD simulations were compared to experimental data using adiabatic effectiveness and thermal field data. Comparison to thermal field data allowed for better understanding of the coolant flowfield above the model. The thermal field measurements were conducted along the stagnation plane and at a plane downstream of the offstagnation row of holes. The author found no previous studies that conducted thermal field measurements on the stagnation plane of a film cooled leading edge model. This is a critical region to the film cooling scheme. 


\section{Chapter 2: Experimental and Computational Setup}

This thesis analyzed film cooling results on both an experimental model and computational simulations. The objective of the study was to compare the CFD predictions of coolant flowfield and surface temperature to experimental data. Thus, the computational leading edge model had similar geometry and flow conditions to the experimental leading edge model. The following sections will first explain the experimental setup, followed by the computational setup of the CFD models

\subsection{Experimental Setup}

The experiments studied in this thesis were conducted on a scaled leading edge turbine blade model. A closed loop wind tunnel facility was used in the experiments with the hot mainstream gases simulated by bulk mass flow of room temperature air inside the tunnel. A schematic of the wind tunnel facility is shown in Figure 2.1. The coolant used for these experiments is cold nitrogen gas. An average mainstream temperature of $300 \mathrm{~K}$ and an average coolant temperature of $200 \mathrm{~K}$ were maintained during the experiments. The facility used in this experimental study was existing and had been used in numerous previous studies such as Albert et al. (2004), Terrell et al. (2004) and Dyson et al. (2010). 


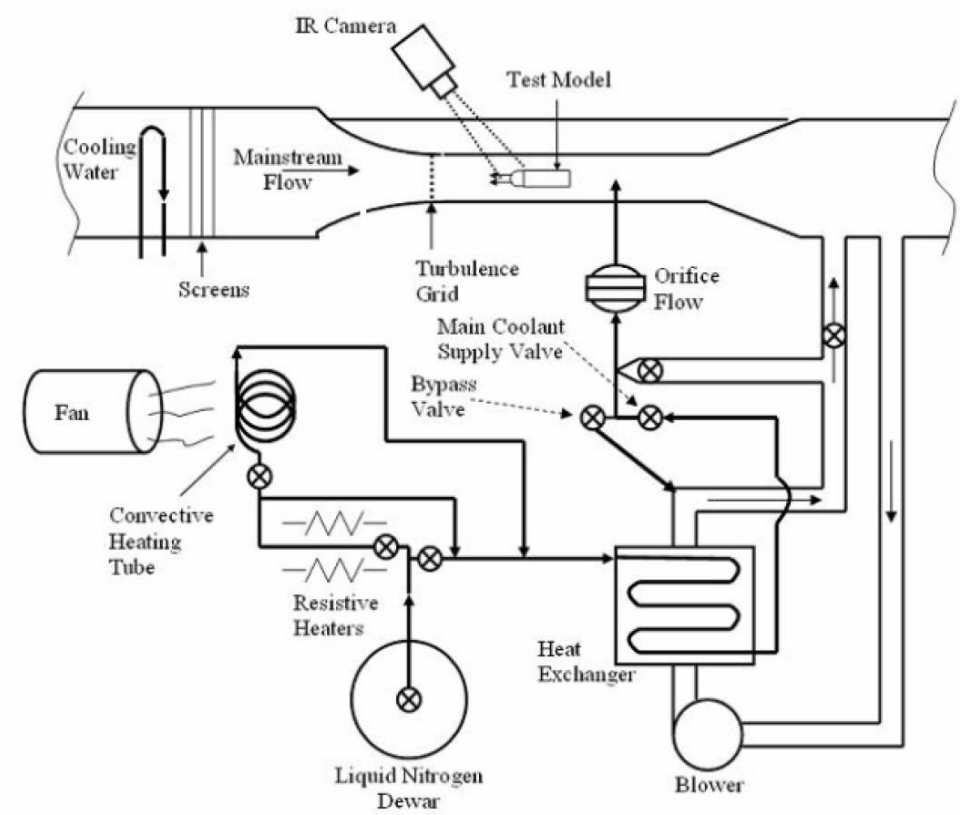

Figure 2.1- Schematic of wind tunnel test section and secondary cooling loop

The closed loop wind tunnel was powered by a $5 \mathrm{hp}$ axial fan. The mainstream gas was also directed through a heat exchanger which is supplied with tap water. This ensured that the mainstream air remained at a constant ambient temperature through the experiment. Downstream of the heat exchanger was nozzle with a 9:1 area contraction in the wind tunnel geometry. The end of the nozzle was directed towards a plexiglass constant cross sectional area test section. Turbulence generators were used to study the effects of turbulence on film cooling performance. The generators consisted of cylindrical vertical bars that measured a diameter of $9.5 \mathrm{~mm}$ and a spacing of $25 \mathrm{~mm}$. The leading edge model was placed within the test section at a distance of $0.38 \mathrm{~m}$ from the turbulence generators. Maikell (2008) indicated the turbulence intensity as $6 \%$ at a distance of $0.38 \mathrm{~m}$ downstream of the grid. At this distance, the integral length scale was calculated to be 19 
$\mathrm{mm}$. It is noted that turbulence levels were not directly measured for experiments studied in this thesis. The test section, where the leading edge model was placed, had a width and height of $61 \mathrm{~cm}$ and $15 \mathrm{~cm}$ respectively. Thermocouples and pitot static probe were also placed within the test section in order to measure the temperature and velocity of the mainstream. During experiments, the mainstream air had a constant velocity of $15 \mathrm{~m} / \mathrm{s}$ and this resulted in a model Reynolds number of 48,000, based on leading edge diameter

\subsection{Coolant Supply}

The coolant used in the film cooling process is cold nitrogen gas. External nitrogen container of $175 \mathrm{~L}$ and $160 \mathrm{~L}$ capacity were attached to a secondary loop. The secondary loop fed into the leading edge model through a plenum system, which will be described later. Liquid nitrogen has a temperature of $77 \mathrm{~K}$ and this is too cold for film cooling simulations. All the experiments were conducted at a density ratio of 1.5 and ideal gas behavior is assumed for both the mainstream and the coolant.

With a mainstream temperature of $300 \mathrm{~K}$ and $\mathrm{DR}=1.5$, the coolant temperature must be maintained at $200 \mathrm{~K}$. The liquid nitrogen at $77 \mathrm{~K}$ is heated to $200 \mathrm{~K}$ by passing it through a flow loop that heats the coolant through convective heating. The coolant nitrogen was also directed through a heat exchanger that used the hotter mainstream air. This heat exchanger was driven by a $7.5 \mathrm{hp}$ centrifugal blower. Along with increasing coolant temperature, the exchanger also helped maintain mainstream air temperature. 
The coolant mass flow rate was measured using an orifice meter that was located upstream of the plenum reservoir and the leading edge. The temperature of the coolant was also measured at the orifice meter. The coolant then entered the plenum reservoir attached to the leading edge model. The plenum section was insulted and reduced temperature increase of the coolant. The temperature of the nitrogen gas was also measured within the plenum. The coolant is finally routed through an impingement plate and the leading edge model to the external model surface.

With the use of room air to simulate mainstream gas and the low temperatures experienced by the coolant nitrogen, it was possible for frost to accumulate within various parts of the leading edge model. The humidity within the air condenses out when in contact with the cold coolant. The accumulation of frost on the surface of the leading edge model can result in lowering of the surface temperature and can also affect the flow field of the coolant upon exiting the coolant hole. Thus for an accurate study of film cooling, it was necessary to eliminate or minimize the accumulation of frost on the surface of the leading edge model. The internal surfaces of the model and the orifice meter used to measure coolant mass flow rate were only in contact with the nitrogen gas coming from the external tank and hence have minimal possibility of frost formation.

Dehumidification of the mainstream air was done in two parts. Firstly, the tunnel air was purged with nitrogen from the external tank. The purging process was done by running the liquid nitrogen through the secondary flow loop until it vaporized. The gaseous nitrogen was then diverted around the leading edge model and entered the test 
section through a port behind the leading edge model. The mass influx of nitrogen caused the warmer and hence less dense air to be pushed out of the wind tunnel and hence resulting in the removal of humidity. The second dehumidification process involved the use of desiccant packs that absorbed moisture from the wind tunnel air. As mentioned earlier, the desiccant packs were placed in the tunnel. The desiccant packs were placed downstream of the leading edge model. In order for effective removal of humidity from the mainstream air, the desiccant packs needed to be preheated before being placed within the wind tunnel. The relative humidity in the air was reduced to about $4 \%$ before data was collected for the experiment. The entire dehumidification process took about an hour and the humidity levels were monitored throughout the experiment.

\subsection{Leading Edge Model}

Since the current study is directed towards measuring film cooling performance on the leading edge of a turbine blade, the model used for the current study was a scaled up model of a typical leading edge turbine blade. As seen in Figure 2.2, the leading edge model consists of a cylindrical leading edge section and a flat section extending behind it. 


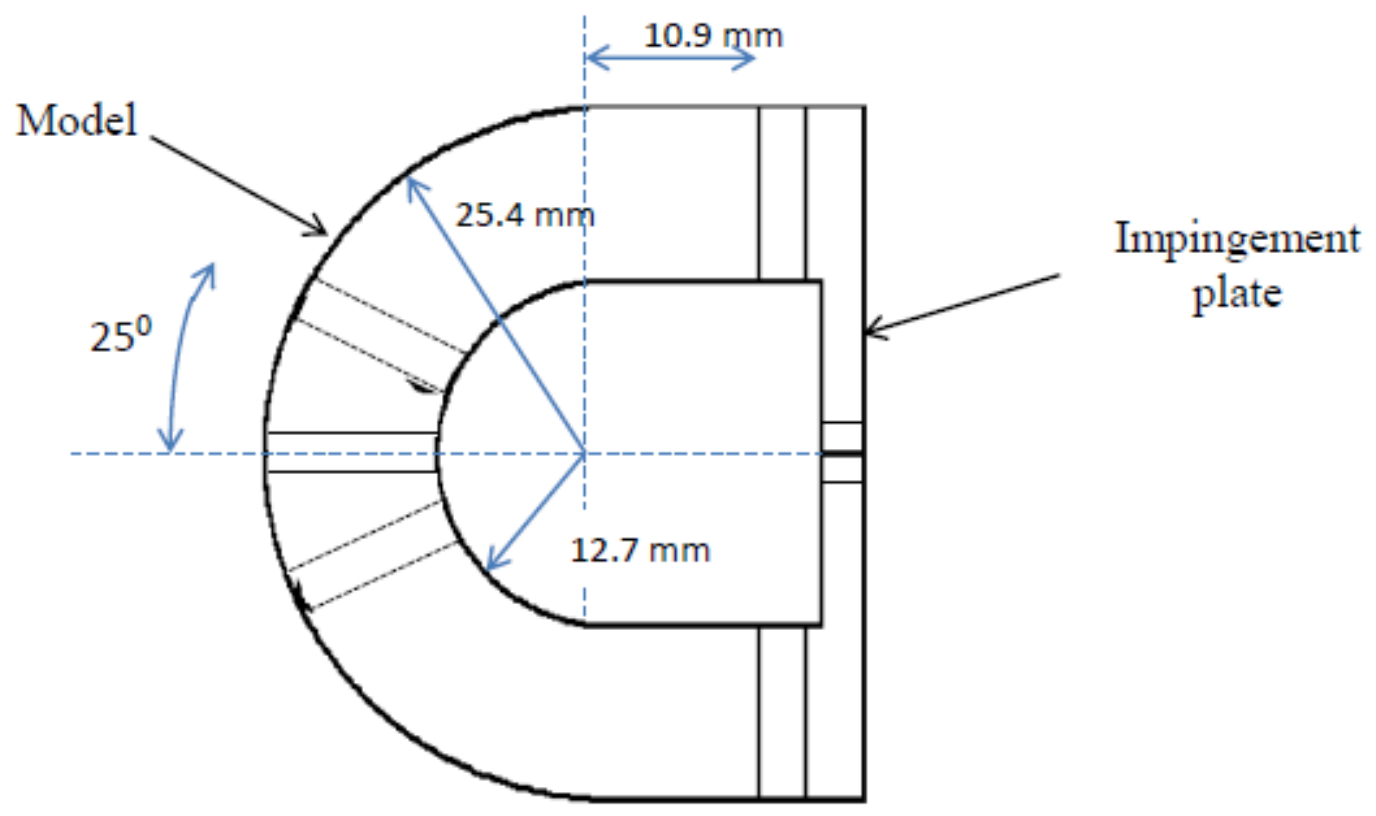

Figure 2.2 - Side view of leading edge model

The cylindrical section consists of a hollow half cylinder with an external radius of $25.4 \mathrm{~mm}$ and an internal radius of $12.7 \mathrm{~mm}$. The flat section extends $10.9 \mathrm{~mm}$ behind the half cylinder and also has a thickness of $12.7 \mathrm{~mm}$. As shown in Figure 2.3, there were three rows of cooling holes located on the cylindrical section, one along the stagnation line and the other at \pm 25 degree from the stagnation plane. The cooling holes have a radius of $3.18 \mathrm{~mm}$ and were located with a pitch to diameter ratio of 7.6 . On the experimental model, each row had a total of 16 cooling holes spanning the model width. The experimental model had a spanwise dimension equal to the spanwise distance of the test section which is $61 \mathrm{~cm}$. The cooling holes studied had an injection angle of 20 degree and a compound angle of 90 degree. The holes were also cylindrically shaped with the stagnation and off stagnation row of hole staggered offset between each other. 


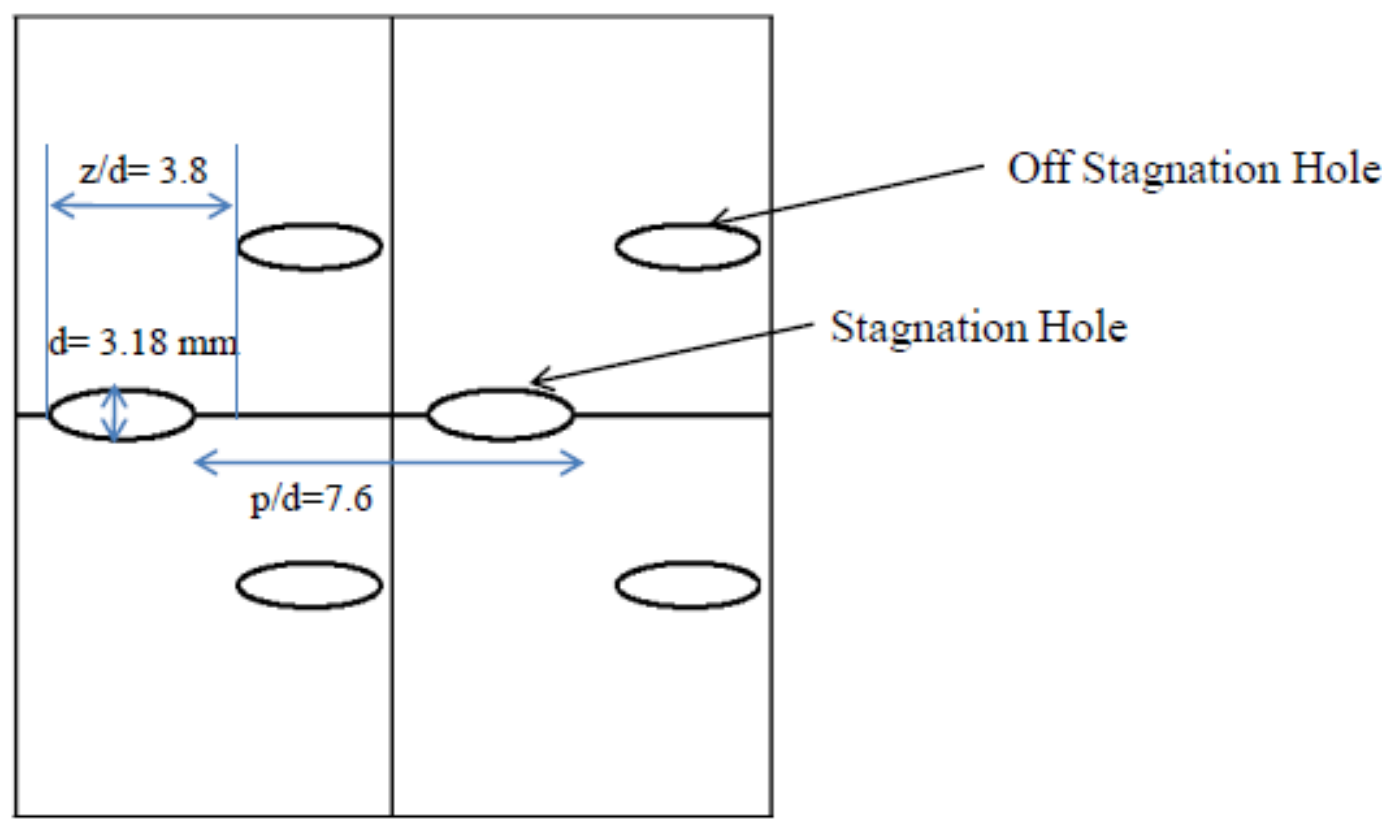

Figure 2.3- Front view schematic of leading edge model

As shown in Figure 2.4, the leading edge model was attached to a plenum section. The coolant was supplied into the plenum and acted as a reservoir for the coolant. The plenum was insulated in order to better control the coolant temperature. The coolant was directed from the plenum to the impingement plate. The impingent plate has hole of diameter of $5 \mathrm{~mm}$ for the impingement hole and directed the coolant towards the internal model surface in the form of high velocity jets. The impingement of coolant onto the warmer internal surface resulted in the increase of convective heat transfer coefficient along the internal model surface.

The average external heat transfer coefficient for the experimental film cooled surface was $h_{f}=147 \mathrm{~W} / \mathrm{m}^{2}$. With typical engine Biot number of $\mathrm{Bi}=1.8$, the thermal conductivity 
of the conducting model equates to $\mathrm{k}=1.04 \mathrm{M} / \mathrm{mK}$. Both the CFD simulations and the experimental had the same thermal conductivity.

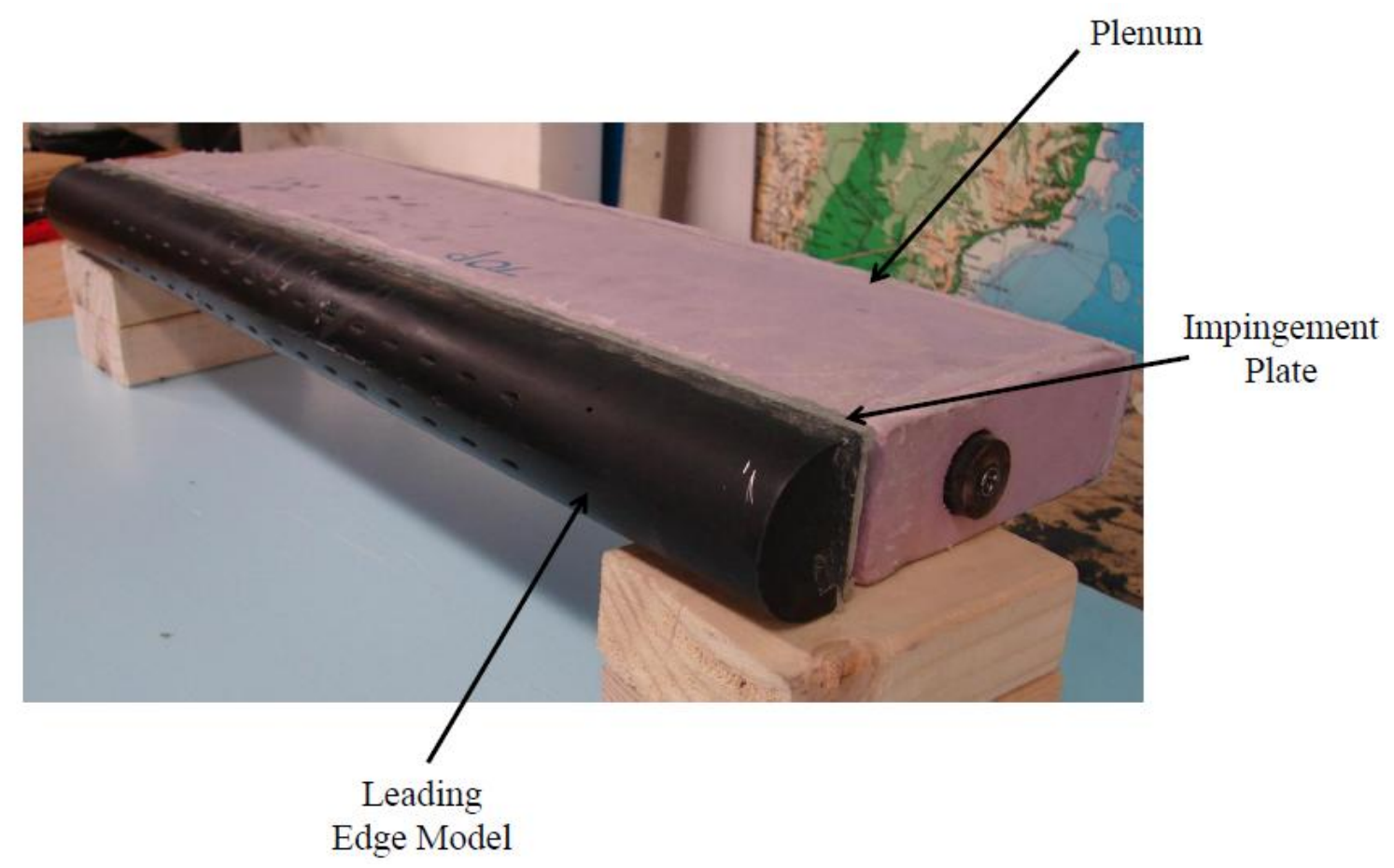

Figure 2.4- Leading edge model with plenum attached

\subsection{Test Section Instrumentation}

All temperature measurements in the wind tunnel were obtained from E-type thermocouples and the flow rates of the mainstream and the coolant were measured using Pitot static probes and orifice meters, respectively.

The mainstream thermocouple was located 10D upstream of the leading edge model along the midspan of the test section. A thermocouple was also located in the flow path of the coolant near the orifice meter that was used to measure the coolant flow rate. 
Additional thermocouples were also located within the plenum near the impingement plate hole. These thermocouples gave the temperature of the coolant at the impingement plate and thus could be used as the internal temperature of the coolant. The density ratio used for the film cooling analysis is calculated based on the temperature of the coolant recorded at the plenum and the mainstream temperature.

A Pitot static probe was located $8.5 \mathrm{D}$ upstream of the leading edge. The static pressure at this location is assumed to be atmospheric pressure. To estimate air density, the Pitot static tube was connected to a Validyne model DP103-16- model pressure transducer, and the signal was analyzed using a National Instrument DAQ system. All experiments were conducted with $\mathrm{V}_{\infty}=15 \mathrm{~m} / \mathrm{s}$.

An orifice meter was used to measure the coolant volumetric flow rate. The orifice meter is described in detail in Albert (2003). The orifice meter had an orifice to pipe diameter ratio ( $\beta$ ) of 0.5 . The pressure drop across the orifice plate was measured using a Validyne pressure transducer, similar to the one used for the mainstream. With the static pressure assumed to be atmospheric pressure and the thermocouple located at the orifice plate, the density of the coolant can be calculated at that location.

With the volumetric flow rate and the density, the mass flow rate of the coolant flowing into the plenum can be calculated. The mass flow of coolant into the plenum is equal to the mass flow rate of coolant out of all the coolant holes on the model. Using conservation of mass, the average mass flux of coolant exiting the coolant hole is as follows 


$$
U_{\text {Jet }} \rho_{j e t}=\frac{m_{c}}{N_{h} A_{h}}
$$

Where $\mathrm{m}_{\mathrm{c}}$ is the mass flow rate of coolant, $\mathrm{N}_{\mathrm{h}}$ is the number of coolant holes on the model,$A_{h}$ is the cross sectional area of each coolant hole and $\rho$ jet is the density of the coolant at the hole exit. With the density of the mainstream $\left(\rho_{\infty}\right)$ and mainstream velocity $\left(\mathrm{U}_{\infty}\right)$ known, the Blowing ratio $(\mathrm{M})$ can be calculated using

$$
M=\frac{\rho_{j e t} U_{j e t}}{\rho_{\infty} U_{\infty}}
$$

All temperature and pressure transducer data was routed real time into the National Instrument DAQ system where the density ratio and the blowing ratio were calculated for every experimental data that was collected for the thesis.

The thermal field measurements used in this thesis required the test conditions to be held at steady $\mathrm{M}=2$ and $\mathrm{DR}=1.5$ for long periods of time. A steady state of the required testing conditions could be maintained for approximately 10 minutes before the DR changed. A heat exchanger, which used a blower described in earlier section, was activated for better control of the DR. The mass flow rate of coolant through the plenum could also be adjusted in order to better control the coolant temperature at the plenum. The adjustment time in order to get back to test condition steady state was approximately five minutes. A typical thermal field measurement experiment lasted six hours. 


\subsection{Thermocouple}

\subsubsection{Thermocouple Conduction Error}

An E- Type thermocouple was used to collect the off the wall temperature profiles along the stagnation and off stagnation row of the film cooled model. The location where the temperature was measured had huge temperature gradients and this could result in a conduction error within the thermocouple wires. Accurate measurement of the thermal field required the conduction error within the probe be minimized.

An energy balance of the probe design can be used to determine the potential for conduction error. With the tip of the probe or the junction being exposed to the local fluid temperature, there is a potential for convective heat transfer between the junction and the fluid. This heat transfer can be found as

$$
Q_{\text {Junction }}=h_{\text {junc }} A_{\text {junc }}\left(T_{\text {fluid }}-T_{\text {junc }}\right)
$$

Where $h_{j u n c}$ is the heat transfer coefficient at the junction, $A_{j u n c}$ is the area of the junction, $T_{\text {fluid }}$ is the local fluid temperature and $T_{\text {junc }}$ is the junction temperature. In ideal conditions, $T_{\text {fluid }}=T_{j u n c}$ and thus the total heat transfer will be zero.

The probe junction is connected to lead wires that that are exposed to temperatures different from the junction temperature. The energy balance of the probe junction also reveals conductive heat transfer within the thermocouple wires. The conductive heat transfer can be measured as 


$$
Q_{\text {cond }}=-k_{\text {wire }} A_{\text {wire }} \frac{d T_{\text {wire }}}{d x}
$$

Where $\mathrm{k}_{\text {wire }}$ and $\mathrm{A}_{\text {wire }}$ are the thermal conductivity and the cross sectional area of the lead wires. $\frac{d T_{\text {wire }}}{d x}$ term refers to the temperature gradient between the probe junction and the lead wires. Even under ideal conditions, for probe used for film cooling measurements, the will be a temperature gradient between the junction and the lead wires as they are exposed to different local fluid temperatures. Thus there will be conductive heat transfer happening across both the lead wires of the thermocouple. Accounting for energy balance, this will ultimately require there be a convective heat transfer at the probe junction to balance the conductive heat transfer within the wire thus resulting in a temperature difference between the thermocouple junction and the local fluid.

\subsubsection{Thermocouple Design}

The thermocouple used for the thermal fluid measurements needed to reduce the conduction error occurring within the probe. A 30 gauge thermocouple wire was used as the primary length of the probe system. This higher thickness wire ensured a secure and sturdy electrical connection to the DAQ system. But the junction of the thermocouple was created using 2-mil wires. Some length of the 2-mil wires were glued onto either side of a sheathed length of the 30 gauge wires and then routed $3 \mathrm{~mm}$ to create the junction. The junction was created by using high conductive thermal paint to join the Chromel and Constantan wires. Thus the probe junction and $2 \mathrm{~mm}$ length of wire that is closest to the 
location of temperature measurement consisted of very thin wire. With most of the thermal gradients being confined to a region close to the surface of the model, the current design ensured that the wires exposed to greatest temperature changes and thus conduction error were the 2-mil diameter wires.

The 30 gauge wires have a cross sectional area of $0.0507 \mathrm{~mm}^{2}$ while the 2- mil wires have a cross sectional area of $0.0020 \mathrm{~mm}^{2}$, a factor of 25 difference. Earlier it was mentioned that the heat transfer within the lead wires is directly proportional to the cross sectional area of the wire. Thus by reducing the cross sectional area of the lead wires and keeping the temperature gradients and the thermal conductivity the same, the heat flow is reduced by a factor of 25 within each lead wire. With reduction in conductive heat transfer within the wires, energy conservation dictates that the convective heat transfers at the junction also decrease. With temperature difference between the probe junction and the local fluid being measured as

$$
\left(T_{\text {fluid }}-T_{\text {junc }}\right)=\frac{Q_{\text {Junction }}}{h_{\text {junc }} A_{\text {junc }}}
$$

a reduction in $Q_{\text {Junction }}$ leads to a decrease in the temperature difference and thus a reduction in conduction error.

Terrell (2004) used a 1 mil wire probe to measure temperatures at the coolant exit hole for a leading edge model. The leading edge model used had similar geometry to the model used in this thesis except that Terrell (2004) had forward diffused shaped holes Measurement of stagnation exit temperatures for adiabatic models with the 1-mil probe 
showed $\theta$ values equal to that at the entrance to the coolant hole, thus proving that conduction error was minimal for the 1-mil probe. Terrell (2004) also measured coolant exit temperatures for a conducting model and showed that for $\mathrm{M}=2$ and $\mathrm{DR}=1.5, \theta$ values at the exit hole centerline was 0.92 . For this thesis, thermal field measurements were also taken at the stagnation plane (hole centerline), but with a 2-mil wire probe. The average stagnation coolant exit temperatures were 0.91 . Since the average value from experiments used in this thesis compares very well with the Terrell (2004) data, it can be concluded that conduction error for the 2-mil probe is minimal.

\subsubsection{Traverse System}

The thermocouple probe was mounted onto a traverse system that was located within the test section of the wind tunnel. The main body of the system consisted of two stepper motors, a pivoting system, a linear stage and rods spanning the test section, as shown in Figure 2.5. It was located behind the plenum of the model in order to reduce the interference of the system on the upstream air and the film cooling flow. The main body of the transverse system was mounted on to a threaded rod of pitch $1 / 16^{\prime \prime}$ that spans the tunnel. Rotation of the threaded rod allowed for movement of the probe in the spanwise direction ( $\mathrm{z}$ direction). This motion was mechanically achieved during the experiment. 


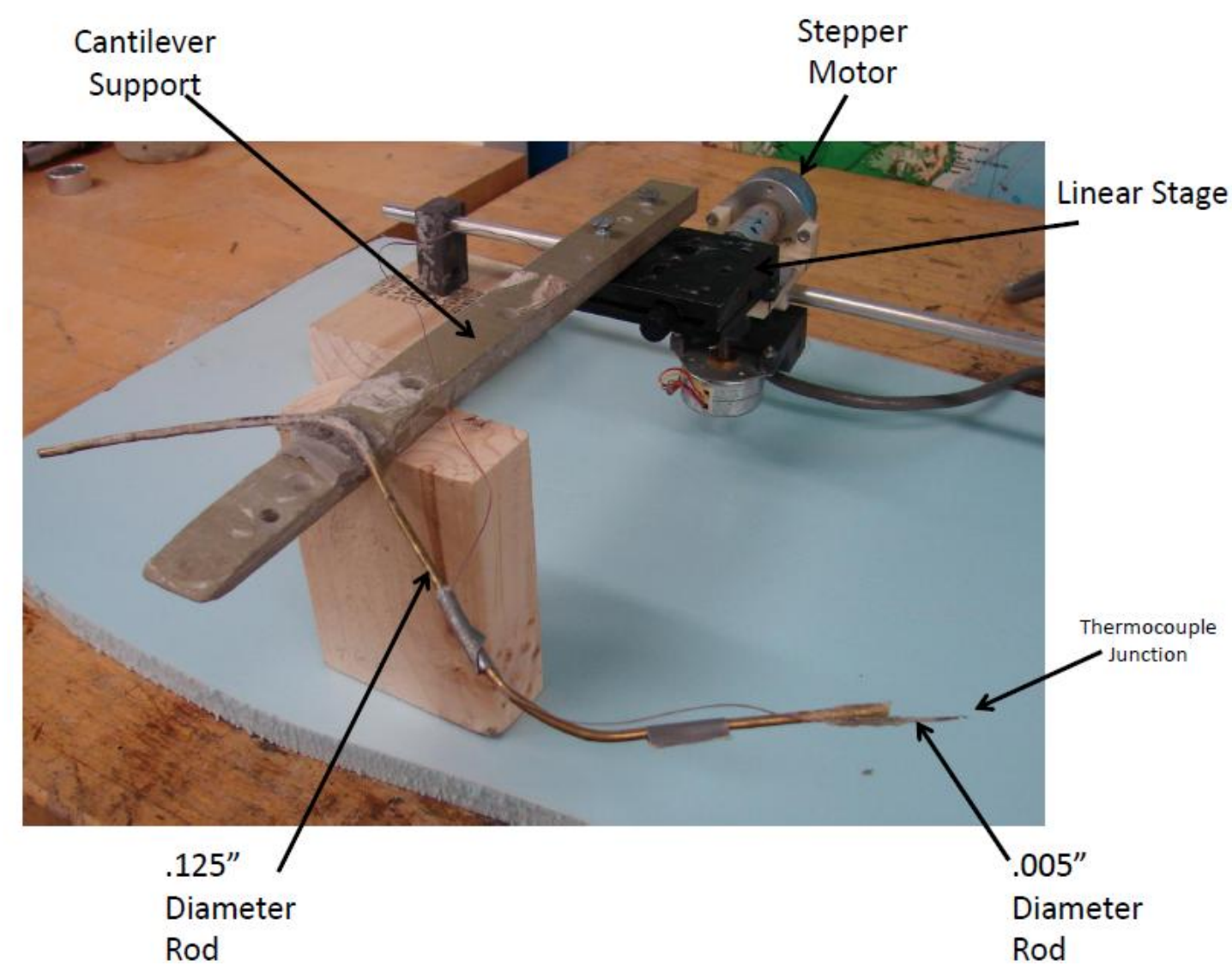

Figure 2.5 - Picture of the Traverse System

As mentioned earlier, the thermocouple wires consisted of a 2-mil junction and a longer section of 30 gauge wire. The thermocouple wires were freestanding for a length of $3 \mathrm{~mm}$ and were then routed through a copper tube of $1.27 \mathrm{~mm}$ diameter. The smaller diameter copper tube was then attached to a larger rod of diameter $3.175 \mathrm{~mm}$. This allowed for minimal flow interference in regions close to the measurement while still maintaining structural stability in the wind tunnel. The larger diameter rod spanned a distance of $215 \mathrm{~mm}$ from the junction, with the base attached onto a cantilever support. A sturdy light weight rectangular beam of dimensions 1 " $\mathrm{x} 0.5$ " was used as cantilever 
support .The $3.175 \mathrm{~mm}$ diameter rod formed a $25 \mathrm{deg}$ angle with the model surface. This is done so that only a small length of the probe system is near the model, with a majority of the probe arm being away from the mixing layers created by the film cooling process.

The direction of the probe arm was also significant to the design of the traverse system. With reference to Figure 2.6, the direction of coolant exit is assumed to be primarily in the negative $\mathrm{z}$ (span) direction and the center of the coolant exit hole is referred to as the origin. With such a design, the length of the probe arm lay downstream of the coolant hole in the spanwise direction and the thermocouple junction is the part of the probe arm that was closest to the coolant exit hole. Thus at the location of temperature measurement, the impact of the probe arm length was minimal.

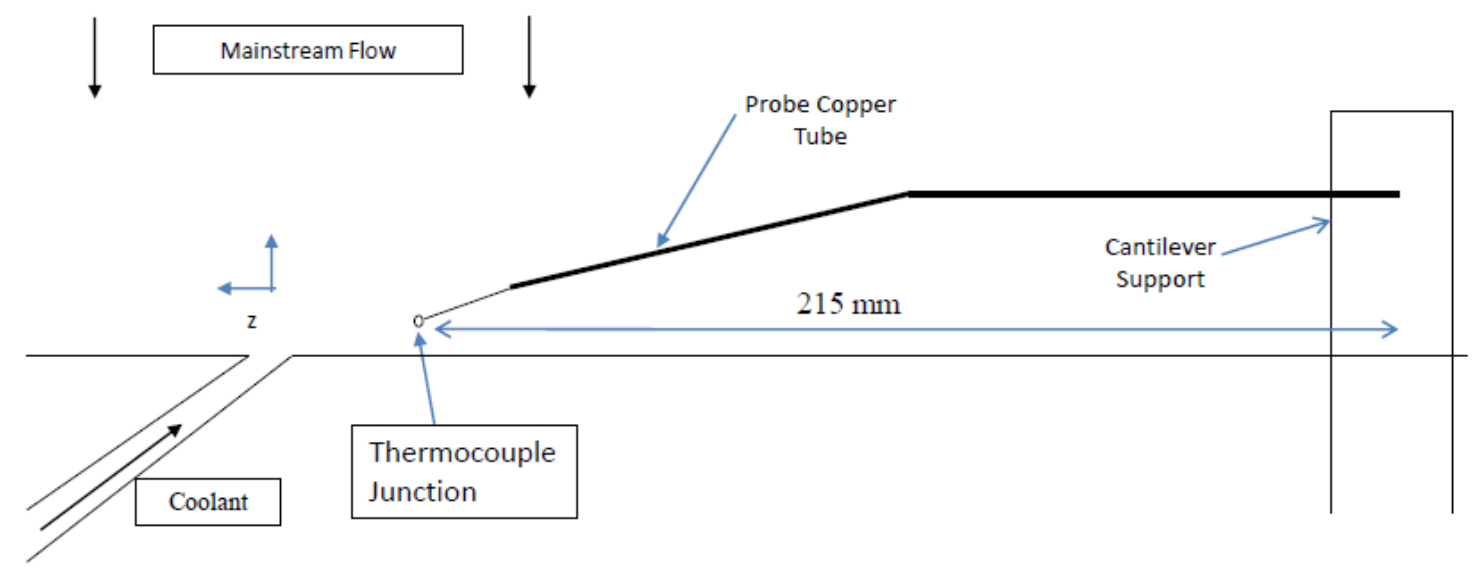

Figure 2.6 - Top view of Stagnation plane. Schematic of the probe junction and probe length with respect to the coolant exit hole

The tip of the thermocouple extended a distance of $215 \mathrm{~mm}$ from the main body of the probe system. This length induces a relatively high moment causing the support to vibrate inside the wind tunnel due to the mainstream impact. But, for accurate thermal 
measurements, the vibrations of the probe had to be significantly reduced. The use of 1 " x 0.5 " cantilever support helped reduce vibration. Also, the threaded rod, on which the main body is mounted, was bolted on to the sides of the wind tunnel to decrease vibrations of the traverse system. While the bigger dimensions of the cantilever support allowed for a sturdier support, it also resulted in a bigger interfere to the mainstream flow. Thus a balance was found between having a sturdy support yet not causing a significant obstacle to the flow field.

The main body of the traverse system consists of two stepper motors. The first stepper motor is attached to the linear stage and can hence traverse the linear stage. Since the probe is attached to the top of a linear stage, the probe can move in a direction parallel to the linear stage, indicated by direction 1 in Figure 2.7. A second stepper motor can pivot the probe system about a pivot point and this direction of probe motion is indicated by direction 2 in Figure 2.7. Both stepper motors are permanent magnet motors, manufactured by ASTROSYN and have a step size of $7.5^{\circ}$ i.e it required 48 steps to complete one rotation of the lead screw of the motor. The stepper motor used for horizontal motion is attached to a linear stage. The linear stage is manufactured by NMC has dimensions of 3"x 3"x 0.75 " and a position screw of $1 / 20$ " pitch. The cantilever support is bolted onto the top of the linear stage. The horizontal stepper motor and the linear stage are placed on a light weight surface that can be pivoted vertically by the second stepper motor. 
This design allowed for the traverse system to have a unit step size of $0.0265 \mathrm{~mm}$ in the horizontal direction. It has a step size of $0.0176 \mathrm{~mm}$ in the pivoting direction.

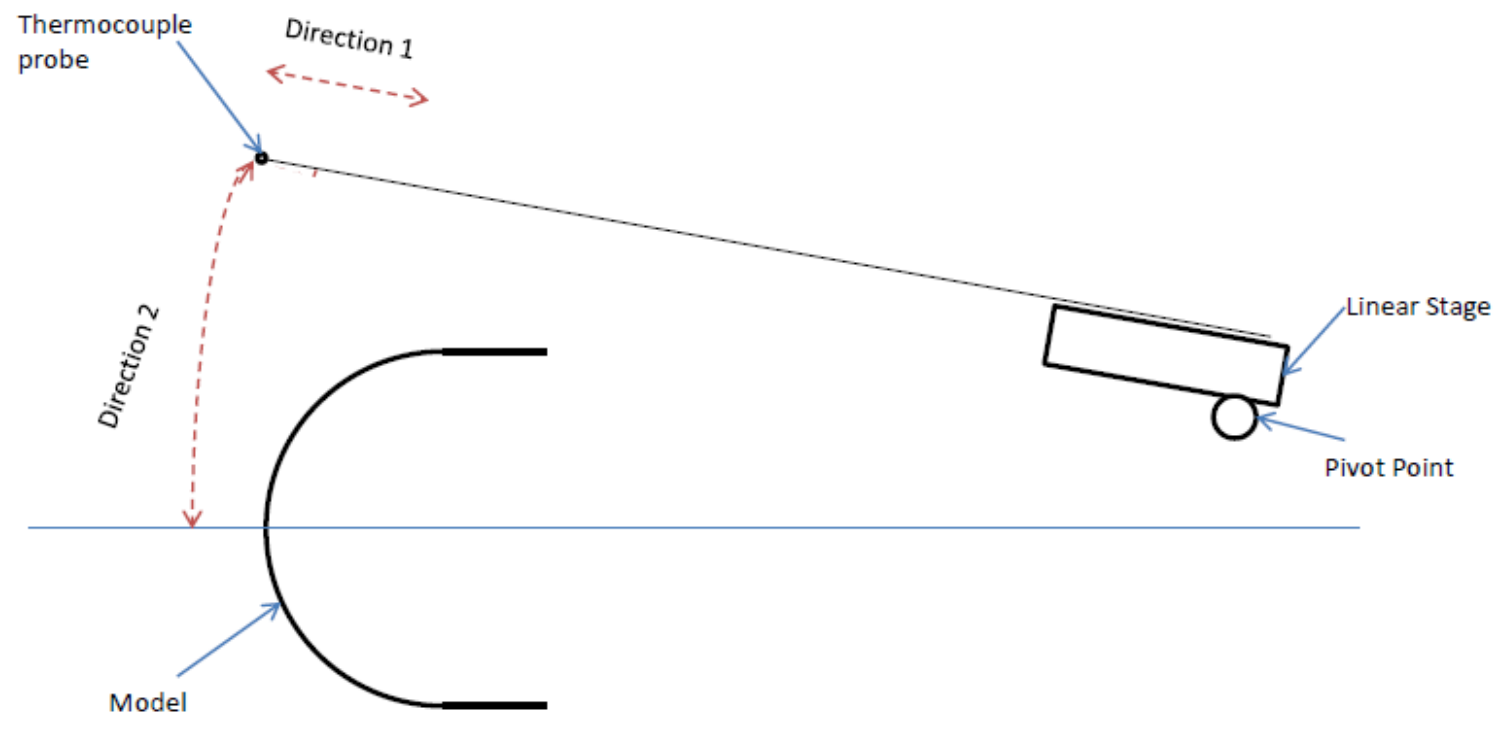

Figure 2.7 - Allowable motion of the probe

\subsubsection{Stepper Motor Software}

The two stepper motors were controlled from outside the wind tunnel. The motors were connected to a stepper motor controller that allows for switching between the two motors and are powered by 12 Volt DC supply. The controller was connected to a NI USB-DAQ 6008 was then connected to the computer. The control algorithm from the computer was relayed to the DAQ as analog signals which were used to control the direction of rotation and the number of steps of the stepper motors.

NI Signal Express was used to control the motors. Analog signals of $\pm 5 \mathrm{~V}$ were used for the motor control. A switching of voltage from 0 Volt to 5 Volt allows for 1 step 
rotation of the stepper motor. With the step motor energized to 5 Volt, switching the motor voltage again to 0 Volt results in another motor step. Thus creating a digital pulse, allows controlling the number of steps or iterations of the motor. The direction of motor rotation can also be controlled by energizing the direction input of the controller. A direction voltage of 0 Volt refers to rotation in the anti clockwise direction while 5 Volt causes motor rotation in the clockwise direction. The direction and step number voltages can be independently controlled.

Figure 2.8 shows the LabVIEW signal express program created by the author used for the stepper motors. DAQ 6008 has two analog input channels with the first channel used for direction control. The second channel experiences switching between voltages for motor stepping. The switching between voltages within the same channel is accomplished by activating the "allow hardware reuse" feature within the execution control tab, as shown in Figure 2.9. The entire cycle of commands is repeated for continued steps of the motor. 


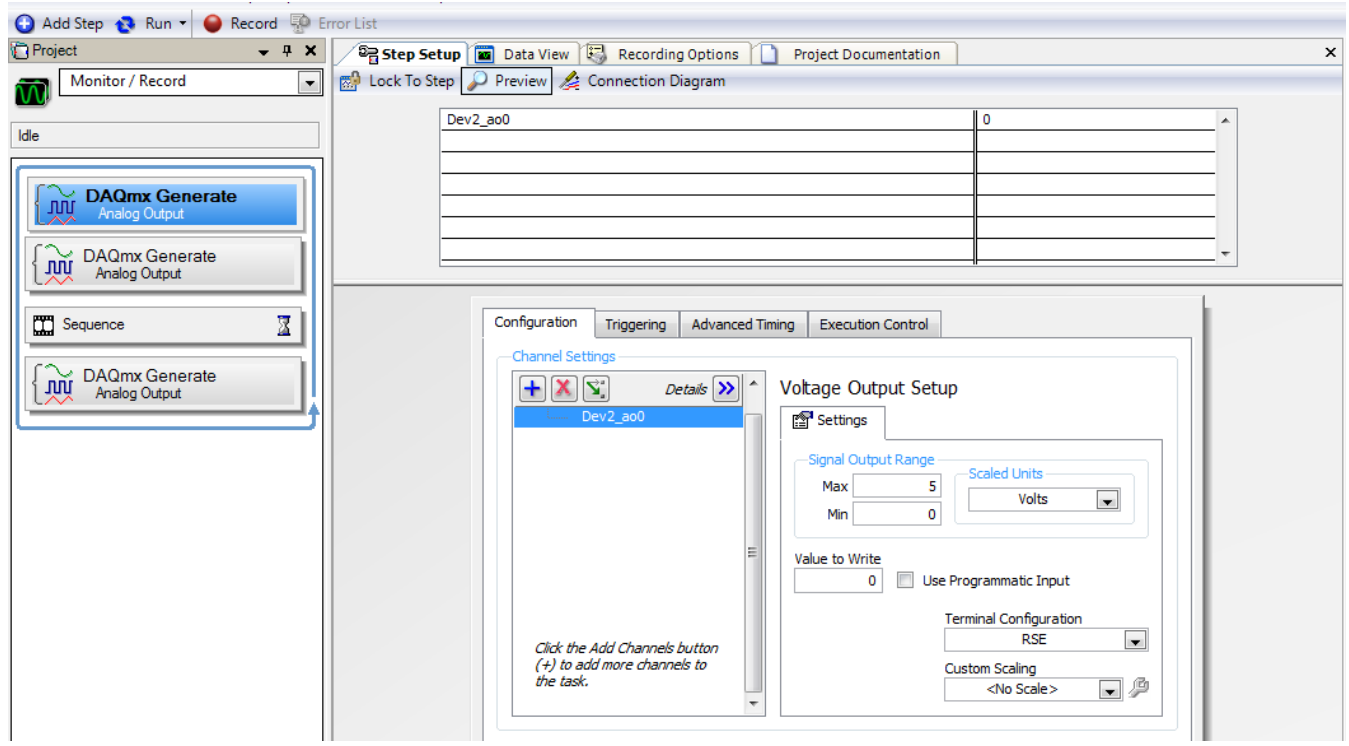

Figure 2.8- LabVIEW Signal Express Code used for stepper motor control

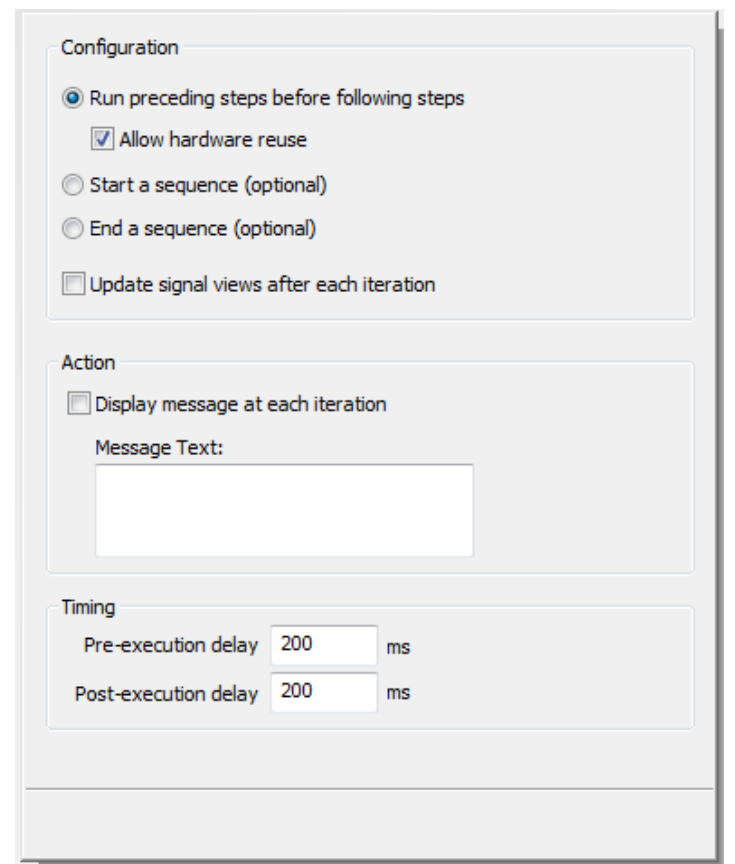

Figure 2.9- 'Allow Hardware reuse' control that allows switching between voltages within the same channel 


\subsection{Experimental Repeatability}

The test to test repeatability was conducted at a nominal $M=2$ and a $\mathrm{DR}=1.5$. The thermal profiles off the wall are measured at the same spanwise location for two separate experiments. For the model, z/d represents distance in the spanwise direction with reference to the lip of the stagnation exit hole, y/d represents distance normal to the surface and $\mathrm{x} / \mathrm{d}$ represents distance downstream of the stagnation line.

For the stagnation plane, test to test repeatability was measured at $2.5 \mathrm{~d}$ from the lip of the stagnation hole. Figure 2.10 reveals that the data is very similar for $y / d<1$. The average difference between $\theta$ values is 0.01 for the near wall, low temperature region. For $y / d>1$, the differences between the profiles become more noticeable with an average difference in $\theta$ value of 0.05 .

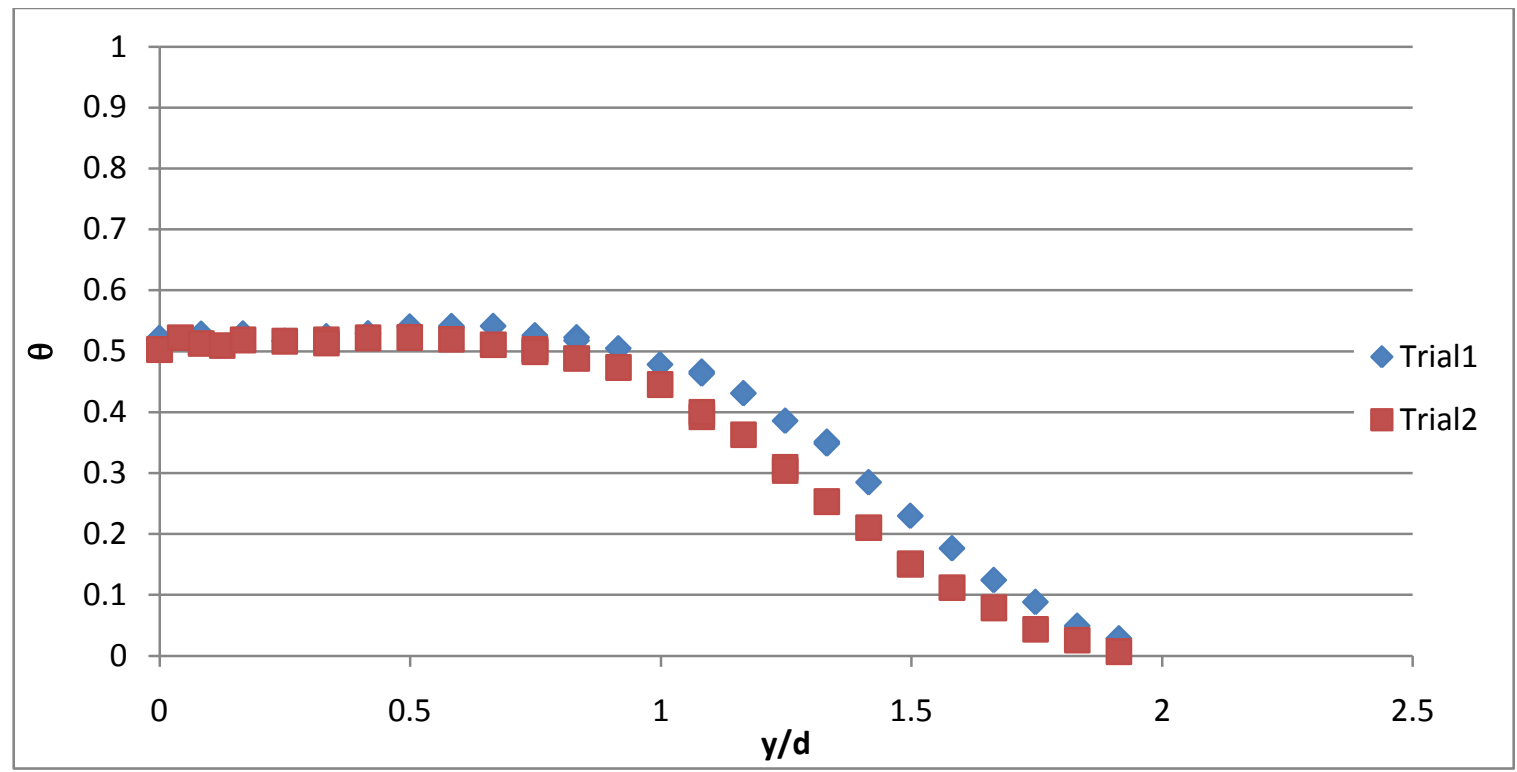

Figure 2.10- Repeatability test at stagnation plane and $\mathrm{z} / \mathrm{d}=2.5$ 
Figure 2.11 represents test to test repeatability at the $\mathrm{z} / \mathrm{d}=7$ position along the stagnation plane. This location is directly above the exit of the stagnation coolant hole. The average difference in $\theta$ value between the repeated profiles is $\Delta \theta=0.024$. This spanwise location showed better repeatability than the $\mathrm{z} / \mathrm{d}=2.5$ location.

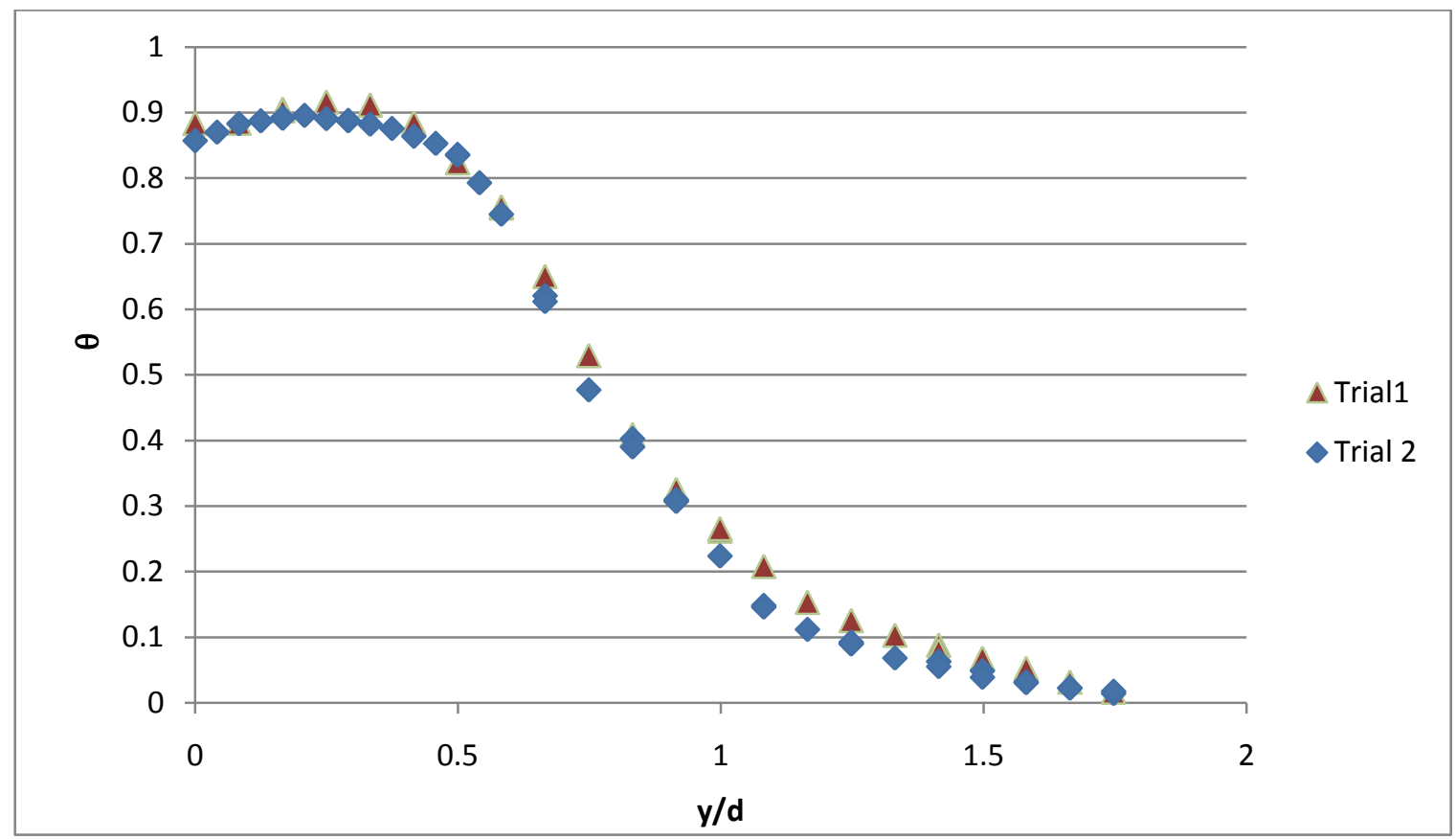

Figure 2.11- Repeatability tests at $\mathrm{x} / \mathrm{d}=0$ and $\mathrm{z} / \mathrm{d}=7$

The coolant exiting the stagnation hole can experience unsteady effects even with steady blowing ratio and density ratio. The effect magnifies at distances further away from the exit of the hole because the core of the jet is narrowed due to diffusion into the mainstream. The narrowed jet is more subject to the counter rotating vortices that can develop around the coolant jet. At $\mathrm{z} / \mathrm{d}=7$, the coolant jet has higher momentum and vortices may not have developed .Thus the bigger difference in profile at the $\mathrm{z} / \mathrm{d}=2.5$ 
location can be attributed to a narrow coolant jet core that is more subject to the unsteady effects.

Offstagnation thermal field measurements were more complex than the stagnation row measurements. While stagnation row experiments used only one stepper motor moving on a horizontal plane, the offstagnation plane was conducted using two stepper motors that were used in tandem to obtain a plane of data perpendicular to the model surface. Figure 2.12 shows test to test repeatability for the offstagnation $\mathrm{x} / \mathrm{d}=5.1$ plane. The spanwise location of coldest coolant temperature was chosen for this analysis. The data shows good agreement with an average difference in $\theta$ value of 0.02 . The thermal profiles were also repeated at location of significant offstagnation coolant separation from the surface (Figure 2.13). This spanwise location experiences significant vorticity of the coolant jet due to interaction with the mainstream fluid. Figure 2.13 reveals that the experiments show good repeatability at this z/d location with an average $\theta$ difference of 0.04 . 


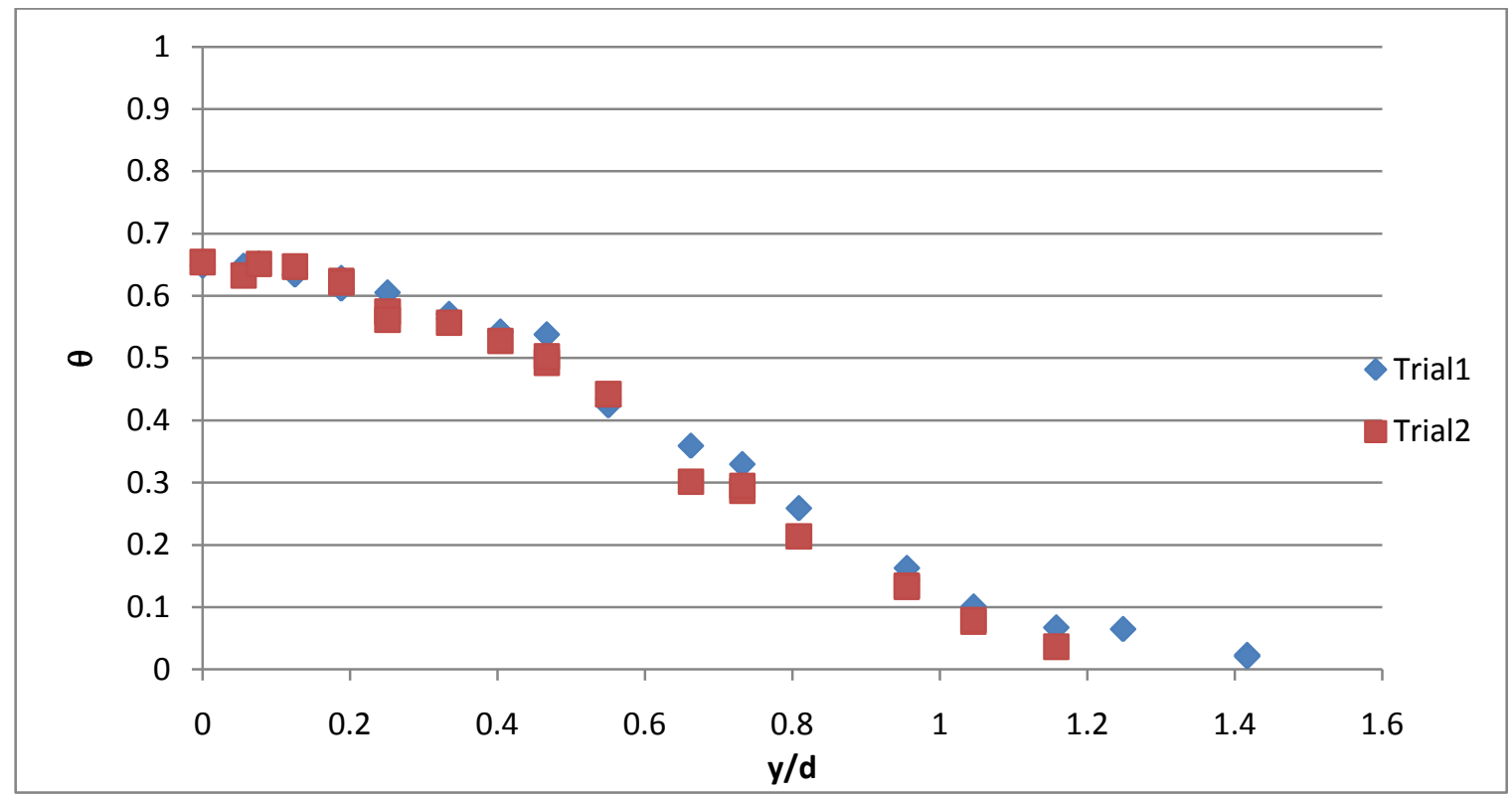

Figure 2.12- Repeatability tests at $\mathrm{x} / \mathrm{d}=5.1$ and $\mathrm{z} / \mathrm{d}=4.9$

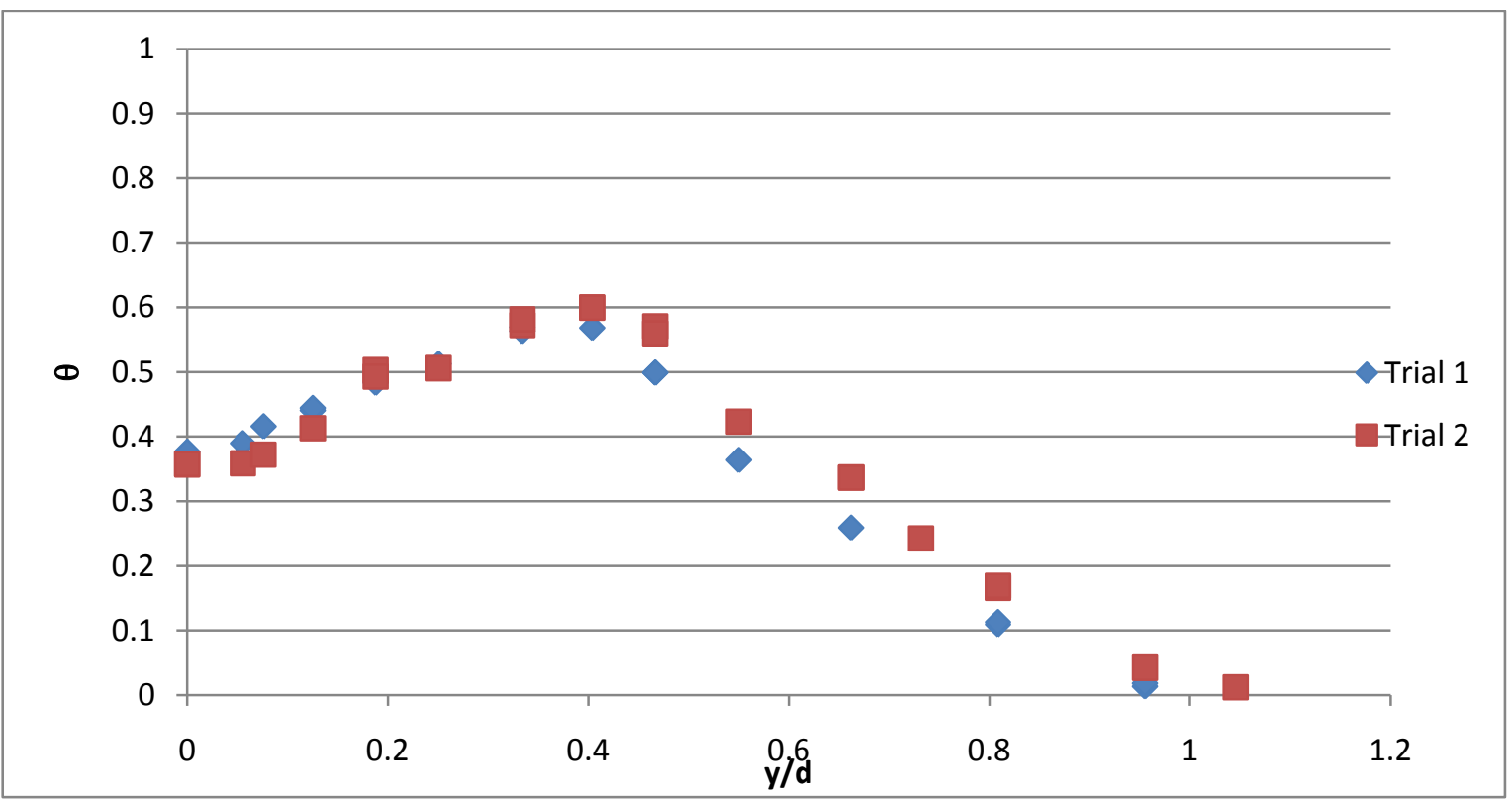

Figure 2.13- Repeatability at $\mathrm{x} / \mathrm{d}=5.1, \mathrm{z} / \mathrm{d}=2.9$ 
The experiments were also compared for in-test repeatability. The data points are taken within a very small time interval for the same spatial location. The average difference between $\theta$ values for in-test repeatability is 0.004 for the stagnation plane while the offstagnation in-test repeatability shows an average difference in $\theta$ value of 0.008 . Thus, the in-test uncertainty is much smaller than the test to test uncertainty indicating that the test to test variation in temperature measurement was probably due to variation in test conditions. 


\section{8 Computational Setup}

This thesis compared the experimental data with the CFD predictions in order to better explain some of the discrepancies between the two results. The leading edge used in the computational study has the same dimensions as the experimental model. The computational mesh used in the CFD study was developed by Ravelli (2009). The leading edge model was generated using Gambit software and was based on the physical model used by Maikell (2008). The computational leading edge model had the same dimensions as the experimental leading edge model, which was described in earlier section. A schematic of the computational domain is shown in Figure 2.14.

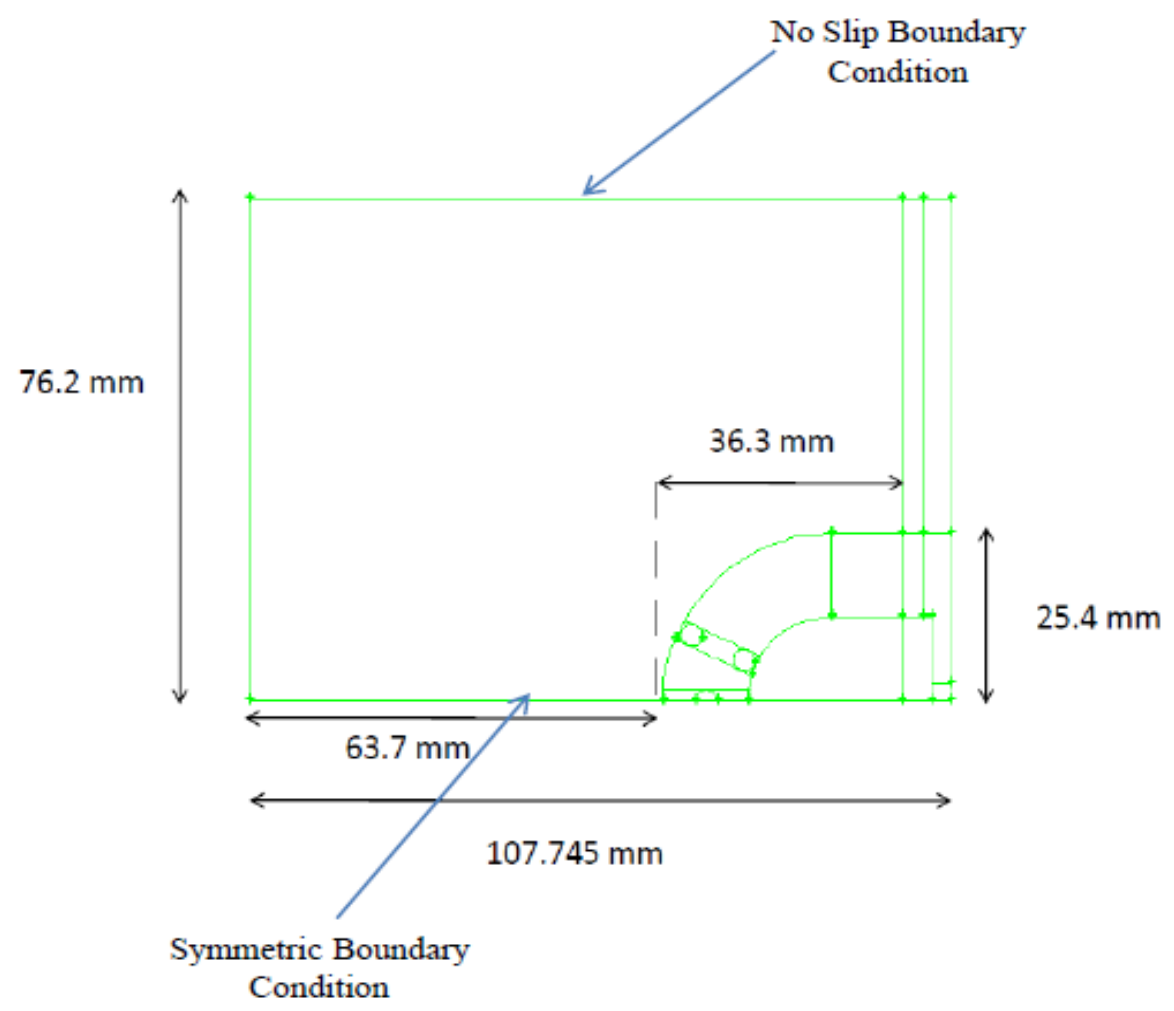

Figure 2.14- Side view of model 
Several computational space and time saving measures were employed within the simulations. Only one pitch distance was computed in the simulations with periodic planes at the sides. In order to take advantage of the symmetric conditions of the model, a symmetric boundary condition was used at the stagnation plane thus enabling simulation of only half the model. The top of the test section was modeled with a no slip boundary condition at a distance of $76.2 \mathrm{~mm}$ from the stagnation plane. The computational domain extended to $63.7 \mathrm{~mm}$ (20d) from the leading edge. The simulations were also tested on a domain that was increased by extending the freestream inlet region further away from the leading edge. The extended domain had the freestream inlet boundary condition located $163 \mathrm{~mm}$ (51d) from the leading edge. It is noted that the extended model was run for an RKE model and did not include an impingement plate; instead a velocity profile was set at the coolant inlet condition. Figure 2.15 compares the overall effectiveness for the extended and smaller domain simulations and Figure 2.16 shows $\phi$ at the stagnation plane. The contour plots show that the temperature distribution is similar for both computational domains. The stagnation line comparison also shows that the two computational grids have similar results. Since the smaller domain mesh showed reliable predictions, the smaller domain was used for all further analysis. 


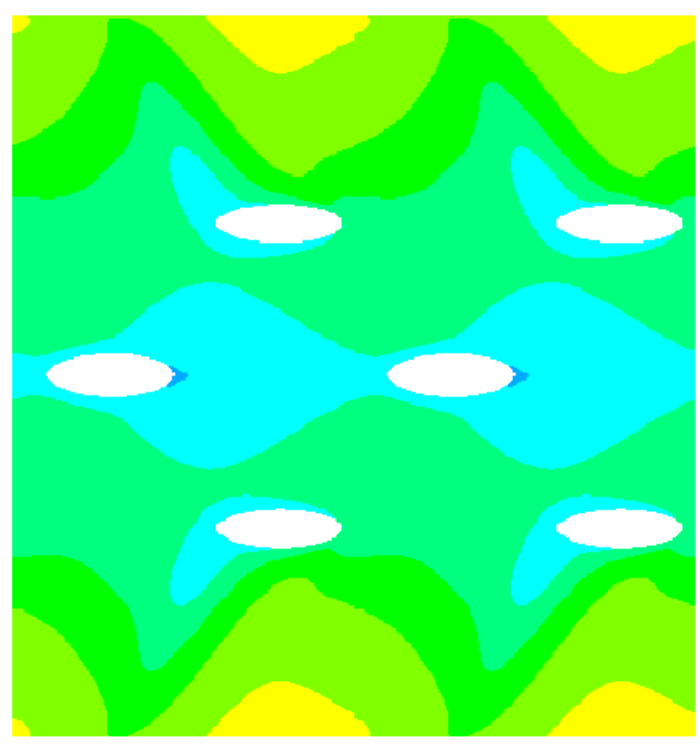

(a) Smaller Domain

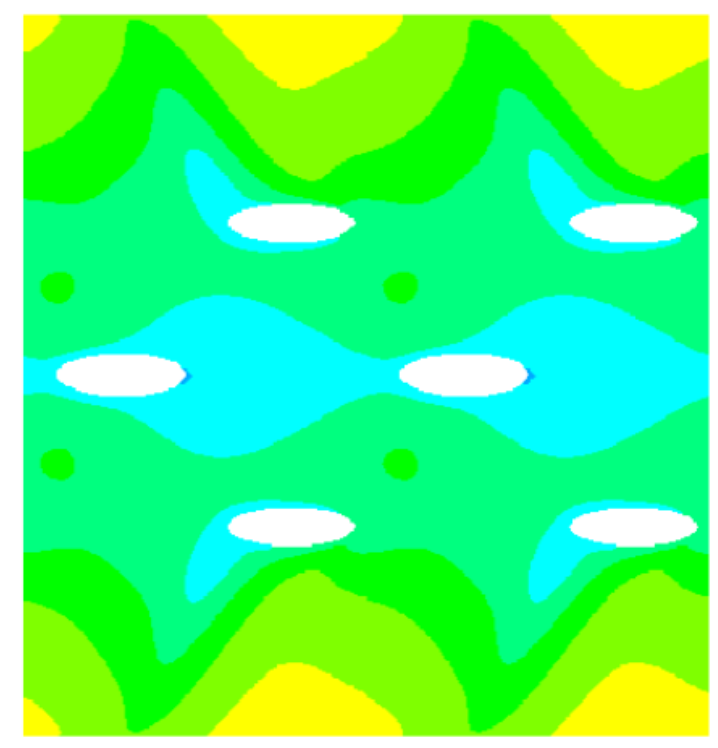

(b) Extended Domain

Figure 2.15- Comparison of Computational domains for RKE model for $M=2$ and $\mathrm{DR}=1.5$

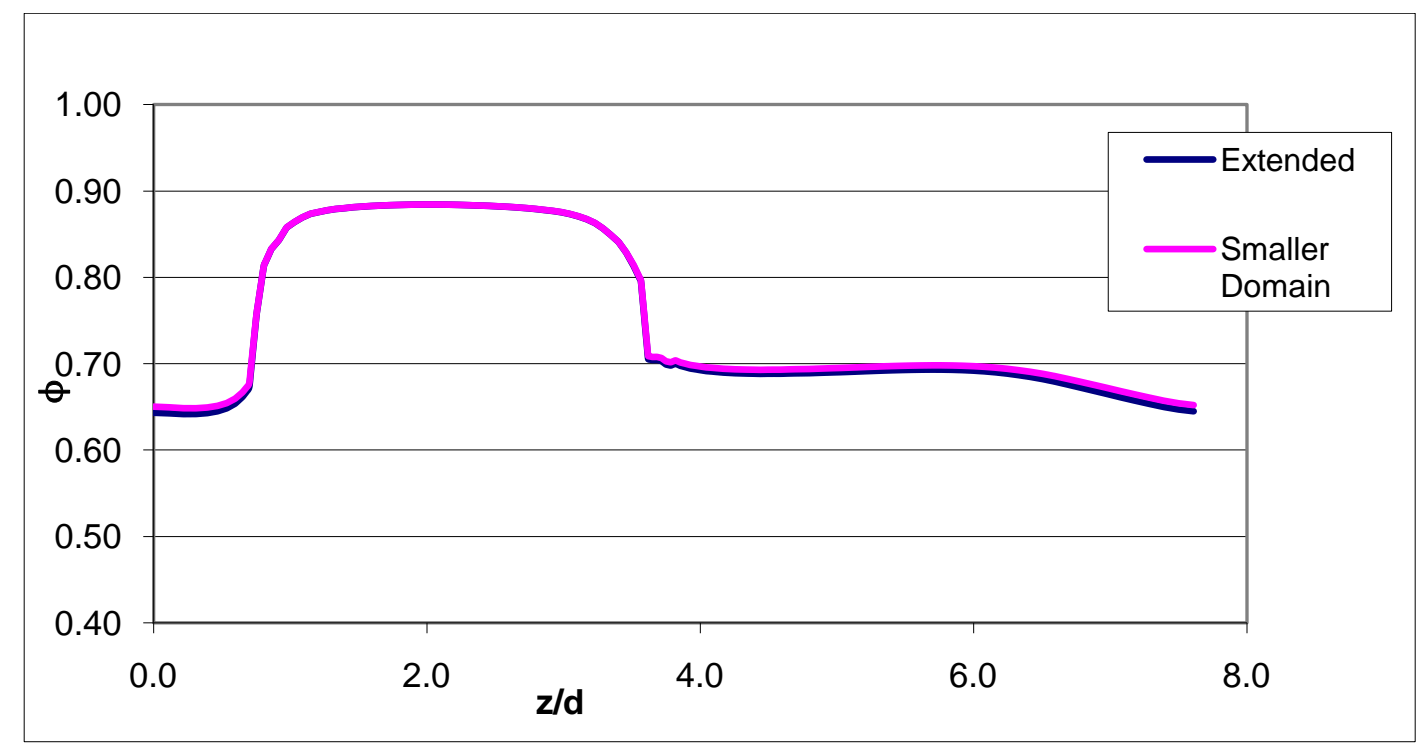

Figure 2.16 - Comparison of $\phi$ along stagnation line with different computational domains 
The freestream inlet temperature was set at $300 \mathrm{~K}$ and the freestream velocity was set at $15 \mathrm{~m} / \mathrm{s}$. A turbulence intensity of $5.9 \%$ and a turbulence length scale of $19.05 \mathrm{~mm}$ was set at the freestream inlet. A mass flow rate input was set at the impingement hole entrance and a value of $0.000411 \mathrm{~kg} / \mathrm{sec}$ was set to obtain an average blowing ratio of 2 . Figure 2.17 shows the computational domain with relevant boundary conditions. The coolant and the freestream used the same working fluid with the working fluid set as air. The density, thermal conductivity and specific heat properties of the fluid were set using temperature dependant polynomial relationships obtained from Mills (2008). The inlet coolant temperature was set at $200 \mathrm{~K}$ resulting in a $\mathrm{DR}=1.5$. The coolant inlet turbulence intensity was set at an arbitrary value of $6 \%$ with a turbulence length scale of $9.5 \mathrm{~mm}$. As noted in Ravelli et al. (2010), a heat transfer coefficient was imposed on the external surface of the impingement plate. The heat transfer coefficient was determined using separate computational simulations and was done in order to better match the experiments, where the impingement plate was constantly exposed to the coolant supply.

The conducting leading edge model has a thermal conductivity of $\mathrm{k}=1.04 \mathrm{M} / \mathrm{mK}$, equal to the experimental model. The adiabatic leading edge model has the same geometry as the conducting model. The adiabatic simulation was done by deactivating the leading edge zone of the computational mesh. The no-slip boundary condition was still retained along the region of solid and fluid interaction. 


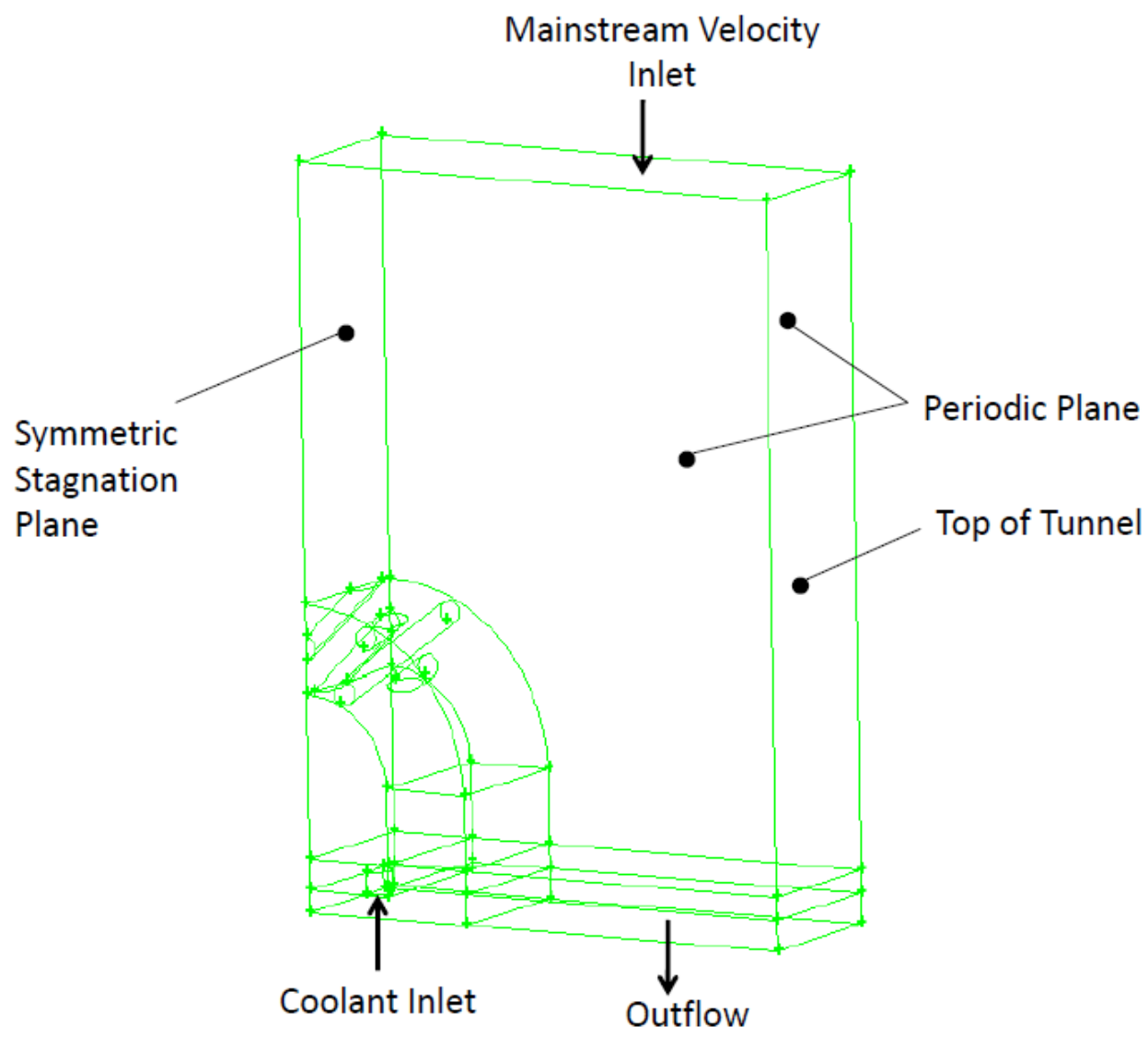

Figure 2.17 - Schematic of leading edge domain

\subsection{Computational Models Specifications}

Several authors have used computational codes to simulate fluid dynamics and hence eventually try better predict film cooling performance. The models used in the CFD runs have varied from flat plate analysis to leading edge models. As mentioned earlier, the current study used the Realizable k- $\varepsilon$ (RKE) model and the Shear Stress Transport (SST) model. 
The RKE simulation used in this thesis was run as node based segregated, steady state and implicit solvers. The use of an implicit solver allowed for higher order of accuracy while reducing the grid resolution needed to achieve converged results. The simulations were started as first order accuracy for the first 300 iterations and were then switched to second order accuracy. This was done in order to avoid large residuals of the solution during the initial iterations while having second order accuracy for the converged final solution. Enhanced wall option treatment was selected for the k- $\varepsilon$ solution along with thermal and pressure gradient effects. Enhanced wall treatment allowed for better resolution into the viscous sub-layer near wall surfaces and resolution of heat transfer effects closer to the wall. The inclusion of thermal and pressure gradient effects allowed for better predicts of the coolant flowfield, lateral spreading and conduction effects.

Similar to the k- $\varepsilon$, the SST k- $\omega$ simulation was run as node based segregated, steady state and implicit solvers. The transitional feature was not enabled because the flow is presumed to remain turbulent due to interaction of the mainstream with the coolant. The under relaxation values for momentum and density were reduced during the initial 300 iterations to reduce initial residual values. The under relaxation factors serve has a factor term that reduces the difference in computed values between successive iterations. They serve to speed up the convergence process.

Both the k- $\varepsilon$ and the $\mathrm{k}-\omega$ simulations used pressure based solvers and a SIMPLE pressure- velocity coupling. 


\subsection{Grid Resolution}

Film cooling on a leading edge model is a complex flowfield that involves interaction between jets and pressure gradient effects. Effects such as coolant separation and jet spreading are usually caused by near wall forces and they need to be accurately predicted. York and Leylek (2002) indicated that resolution of coolant separation from the model surface is an important factor that would explain some of the differences between computational and experimental film cooling results. Thus, it is necessary for a valid CFD simulation to account for near wall effects.

High grid resolution was necessary in order to better capture near wall effects acting on the flow field. With a no slip condition imposed on all wall boundaries, high Reynolds number flow results in the creation of a viscous sub layer directly above the wall surface. The viscous sub-layer is a near wall zone which is dominated by viscous forces and transportation occurs mostly due to viscous diffusion. Capture of this viscous sub-layer is essential for predicting the growth of the coolant separation, heat transfer from the wall and lateral spreading of the jet. The viscous sub-layer can be captured by the Fluent simulation by having node point inside the viscous region. Since the sub-layer has a thickness of $\mathrm{y}^{+}=5$, the first node above the wall is strategically placed very close to the surface to ensure that it has a $\mathrm{y}^{+}$value on the order of one. Also, the RKE turbulence model was selected with enhanced wall functions and this required high near wall resolution. Figure 2.18 shows the mesh along the symmetric plane for the two turbulence models. 
The k- $\varepsilon$ mesh was developed in by Ravelli (2009) using GAMBIT software. The mesh had a total of 4.8 million cells with significantly large number of nodes located at the regions of solid and fluid interaction. Along with small grid spacing in the line and face meshes, a prism layer mesh was applied across the outer layer of the model surface, extending from the symmetric stagnation plane to the top of the impingement plate. The first cell was located at a distance of $0.01 \mathrm{~mm}$ from the surface and had a growth rate of 1.2. The prism layer mesh extended for 20 rows from the surface. Similar prism layers are also assigned to the inner surface of the model and the inner walls of the impingement plate. Due to the curvature of the model surface and the design of the coolant exit holes, it was not possible to create a structured mesh across the entire computational domain. While the above mentioned prism layer regions used a structured volume mesh, the region further away from the walls uses unstructured tetrahedral volume meshes. This allowed for better resolution of the flow field while not increasing the number of nodes and thus giving a reasonable computational time.

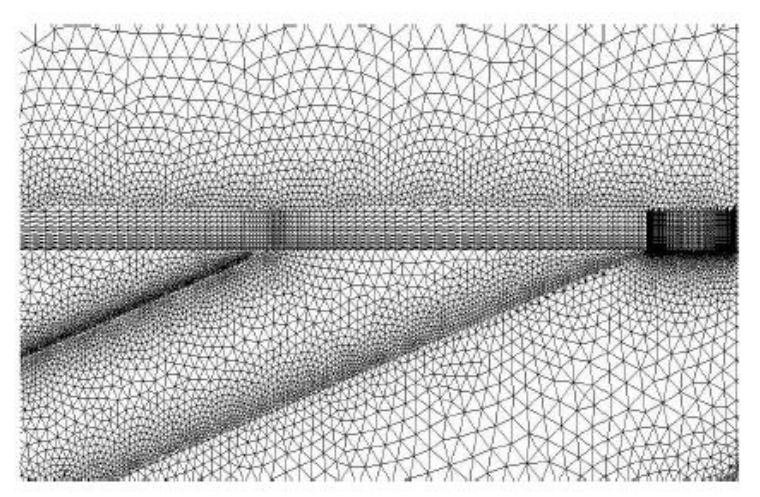

(a) $k-\omega$

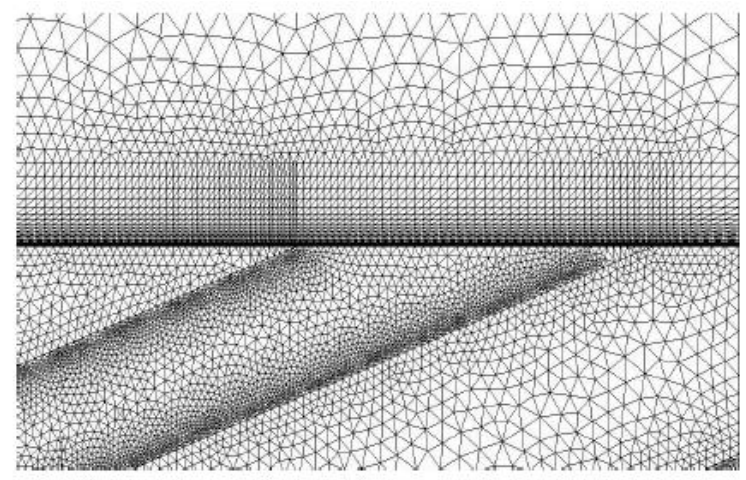

(b) $k-\varepsilon$

Figure 2.18- Grid resolution for the stagnation plane- (a) k- $\omega$ and (b) k- $\varepsilon$ 
The k- $\omega$ mesh was also designed in Gambit software by Ravelli (2009). The simulation used a mesh with 10 million cells. Again structured prism layers were assigned to the external and internal surfaces of the model allowing for the creation of a structured and more accurate mesh closer to the wall. The first node of the k- $\omega$ boundary layer mesh was located at $0.05 \mathrm{~mm}$ from the surface and had a growth rate of 1.1. The boundary layer mesh extended for 10 layers from the surface. Thus the k-w has a lower prism layer thickness compared to the $\mathrm{k}-\varepsilon$ mesh but it is thick enough o contain the coolant trajectory at the coolant exit hole .The k- $\omega$ mesh had a $y^{+}$value of 4 along the model. While the $\mathrm{k}-\omega \mathrm{y}^{+}$value is greater than that of $\mathrm{k}-\varepsilon$, it is still within the viscous sub layer region. Also, Fluent Manual (2009) states that the k-w turbulence model, without transitional features, does not require the first node point off the wall to have a $y^{+}<1$ as long as it is within the viscous sub layer. Figure 2.18 also shows that the unstructured mesh near the wall differs between the two meshes. The k- $\omega$ mesh was created with higher grid density closer to the coolant exit. Also, in the freestream direction, the edges of the k- $\omega$ mesh had more grid points spaced closer to the model surface.

\subsection{Convergence and Grid Independence}

Residuals are the ratio of the difference between two successive iterations to the first iteration. All simulations were initialized using the freestream inlet conditions and since the converged solution differed significantly from the initial guess, it is possible for the residuals to be artificially low. The current simulation used low residual values for

convergence criteria. The residuals were kept below $10^{-9}$ for energy and below $10^{-4}$ for $\omega$. 
All other residuals were kept below $10^{-6}$. At convergence, the surface temperature at a point of high temperature gradient changed by no more than $0.01 \%$ for atleast 200 iterations. Grid independence for the RKE model was tested by Ravelli et al.(2010) and was established by running the simulation with refined mesh of 9.1 million cells, with higher resolution in prism layer. The laterally averaged value of overall effectiveness was very similar. The refined mesh used for grid independence did not have an impingement plate and instead relied on a velocity profile as the coolant inlet condition. 


\section{Chapter 3: Results and Discussion}

This chapter compares the CFD simulations using the RKE and SST turbulence models with experimental data for the leading edge model. The surface temperature contour plots are compared for an adiabatic model in order to analyze coolant flow field. Then, thermal field measurements were analyzed at the stagnation plane and at $\mathrm{x} / \mathrm{d}=5.1$ downstream location. Predictions of coolant separation, mixing with the mainstream and turbulence characteristics are compared with the experimental measurements.

\subsection{Adiabatic Surface Temperature}

The adiabatic surface temperature is widely used to quantify the performance of film cooling schemes. Lower surface temperature indicates regions that are better protected from the effects of hot mainstream gases due to the exiting coolant. The high temperature regions are more prone to failure due to high thermal loads.

The following section compares the surface temperature of the adiabatic model obtained using the k- $\varepsilon$ and the $\mathrm{k}-\omega$ turbulence simulations. Adiabatic model helps better understand coolant flowfield as the surface temperatures are influenced only by flowfield and not conduction effects. The two turbulence models had significant differences in predicted temperatures especially along the stagnation row and regions downstream of the offstagnation hole. The surface temperature is expressed using the adiabatic effectiveness $\eta$. 
Figure 3.1 shows the differences in surface contours of $\eta$ for the CFD simulations and experimental data for the simulated blade leading edge. The experimental surface contours are from Dyson et al. (2010) for the same leading edge geometry. Major differences will be analyzed along the stagnation row, the region between the stagnation and offstagnation rows and, the regions downstream of the off stagnation row of holes. Figure 3.2 compares the laterally averaged value of adiabatic effectiveness for the leading edge. All z/d spanwise distances are with respect to the stagnation exit hole lip, $\mathrm{x} / \mathrm{d}$ is downstream distance with respect to the stagnation plane and $y / d$ is the distance off the wall.

Focusing on the stagnation line of the models in Figure 3.1, the k- $\varepsilon$ has highest $\eta$ values close to the exit of the hole and then continues to decrease in value throughout the span length. Meanwhile, the k- $\omega$ simulation has lower $\eta$ closer to the exit hole, with highest $\eta$ values occurring closer to the leeward edge of the adjacent hole. Such $\eta$ behavior is indicative of the $\mathrm{k}-\omega$ simulation experiencing coolant separation while the $\mathrm{k}-\varepsilon$ stagnation coolant stays attached to the surface even at the exit hole. The k- $\omega$ coolant reattaches at a further distance in the spanwise direction. The experimental contour in Figure 3.1 indicates lower $\eta$ value in region close to the stagnation hole exit. Thus the experimental data show coolant separation. The separation issue is discussed in detail in a later section.

Even through the CFD turbulence models differ in predictions of coolant separation, the laterally averaged $\eta$ value at the stagnation line is similar for the $k-\varepsilon$ and $k-\omega$ model 
(Figure 3.2). Also, the experimental laterally average $\eta$ is lower than both computational calculations. This observation for the RKE model is consistent with the results of York and Leylek (2002) for M=2 and DR=1.5.

The surface temperature contours also show differences in temperature in the region between the stagnation and off stagnation row of holes. This region experiences coolant flow coming from the stagnation row and thus the lateral spreading of the coolant is important in understanding the temperature distribution. The arrows in the CFD profiles for Figure 3.1 indicates the location of highest theta moving downstream of the stagnation row. It is clear that for the $\mathrm{k}-\varepsilon$ case coolant from the stagnation line holes turns in the downstream direction closer to the exit hole. Meanwhile, for the k- $\omega$ case coolant from the stagnation line holes preserves its momentum in the spanwise direction for further distances away from the exit hole before moving downstream. The laterally average $\eta$ value present in Figure 3.2, show that over the $0.5<x / d<3$ region, the $\eta$ values for the $\mathrm{k}-\omega$ case is higher than for the $\mathrm{k}-\varepsilon$ case by $30 \%$. Comparison with experimental result shows that $\eta$ from the $k-\varepsilon$ predictions match the experiments well but the $\eta$ from $k-$ $\omega$ predictions are $30 \%$ too high. 

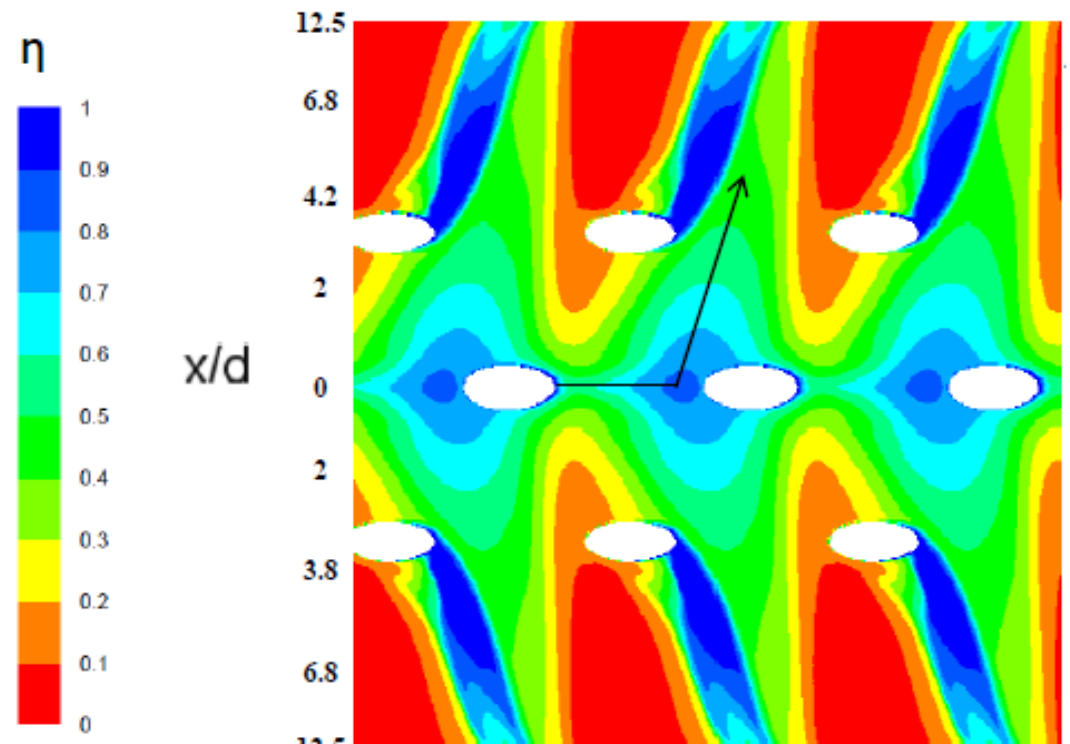

(a) k-w

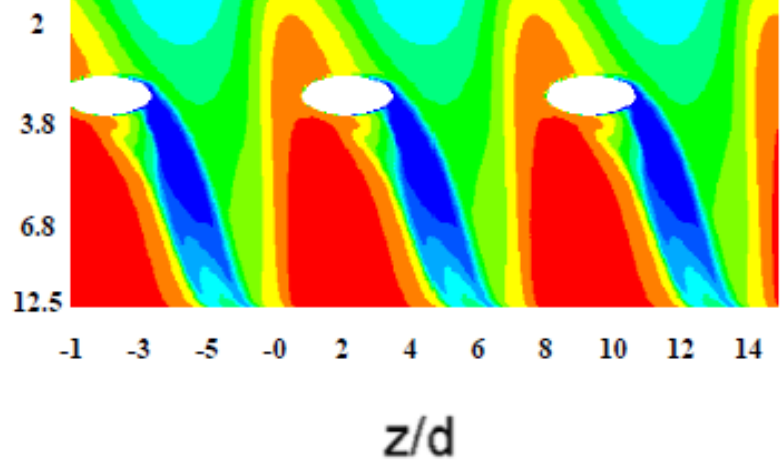

(b) k- $\varepsilon$
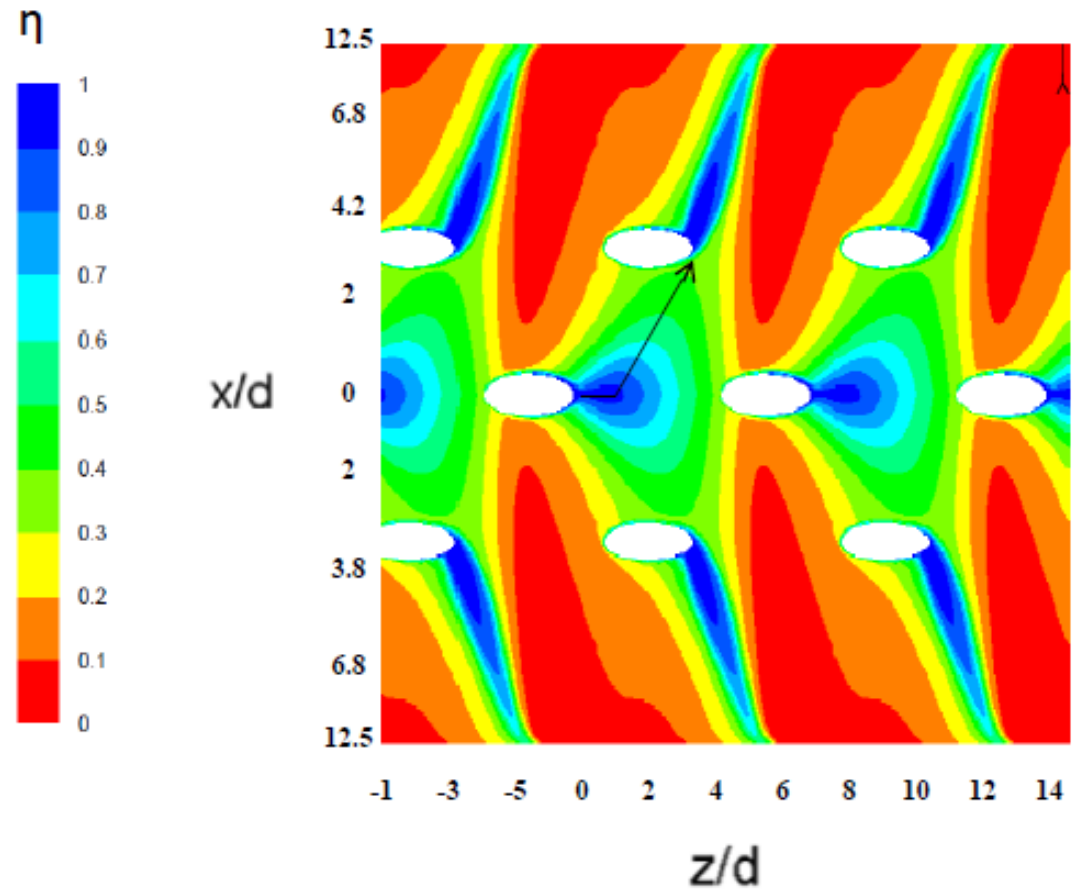


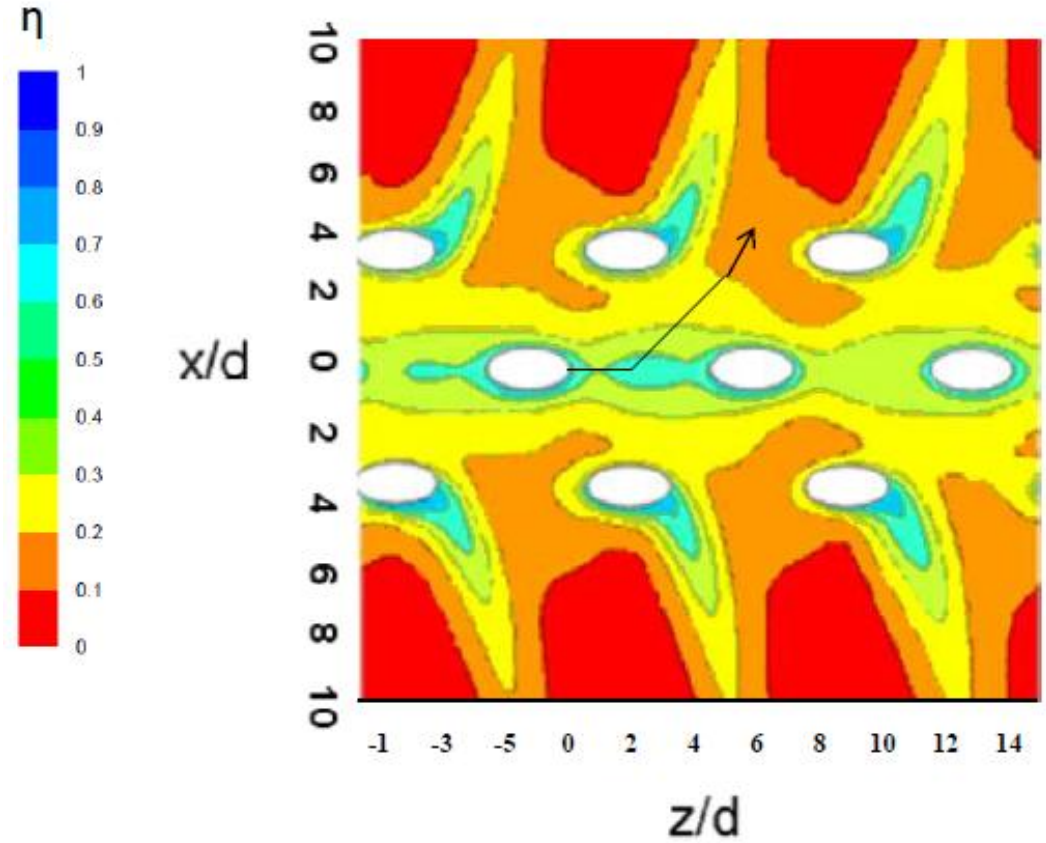

(c)

\section{Experimental}

Figure 3.1- Contours at $\mathrm{M}=2$ and $\mathrm{DR}=1.5$ - (a) $\mathrm{k}-\omega$, (b) $\mathrm{k}-\varepsilon$ and (c) Experimental

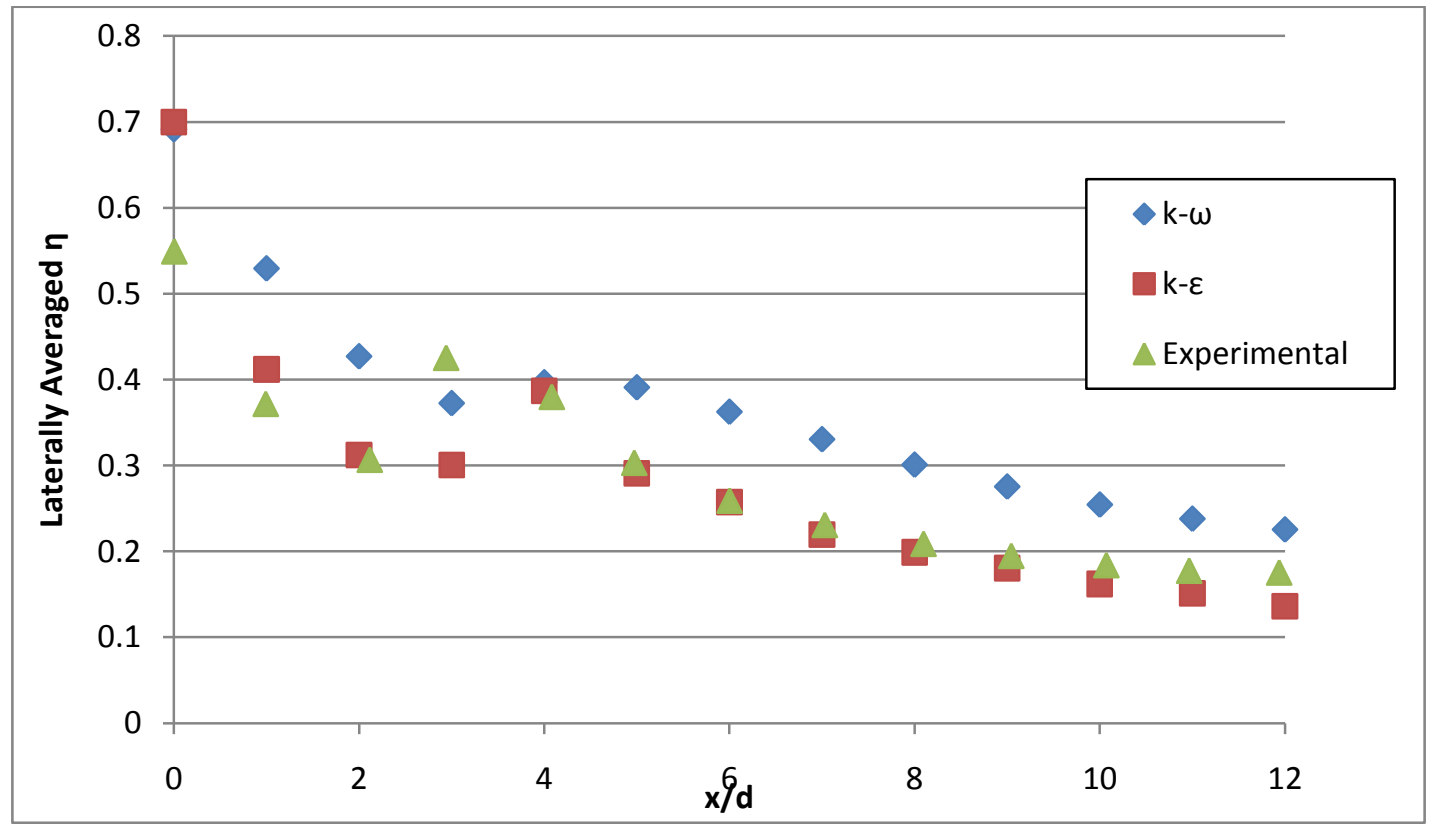

Figure 3.2- Laterally Averaged $\eta$ for the leading edge model for $M=2$ and $\mathrm{DR}=1.5$ 
The different coolant flow paths also indicate different stagnation coolant interaction with the offstagnation coolant. Figure 3.3 compares the k- $\varepsilon$ and k- $\omega$ predictions of $\eta$ profiles at $\mathrm{x} / \mathrm{d}=5.1$ location downstream of the stagnation row. With the peak $\eta$ values indicating the presence of coolant exiting the offstagnation row, both turbulence models show peak values at similar spanwise location. But for $\mathrm{z} / \mathrm{d}>5.4$, the profiles show significant variation between turbulence models. The higher $\eta$ values at this location for $\mathrm{k}-\omega$ predictions, indicates the presence of coolant coming from the stagnation row while the higher $\eta$ values for $k-\varepsilon$ are centered over the coolant from the offstagnation holes. Analysis of the coolant flow path for k- $\varepsilon$ in Figure 3.1 indicates that stagnation coolant joins the offstagnation coolant close to the offstagnation exit hole $(\mathrm{z} / \mathrm{d}=4)$. Thus, the $\mathrm{k}-\varepsilon$ stagnation coolant combines with the core of the offstagnation coolant exit and the combined jet moves downstream in a narrow path. But the $\mathrm{k}-\omega$ stagnation coolant reaches the $\mathrm{x} / \mathrm{d}=5.1$ location at a further spanwise distance from the offstagnation exit hole $(\mathrm{z} / \mathrm{d}=5.4)$ and thus the combined stagnation and offstagnation coolant jets are spread across a greater span length of the model. The greater spreading of the coolant for k- $\omega$ in the region downstream of the offstagnation row results in higher laterally average $\eta$ values for $\mathrm{x} / \mathrm{d}>4$, as shown in Figure 3.2 


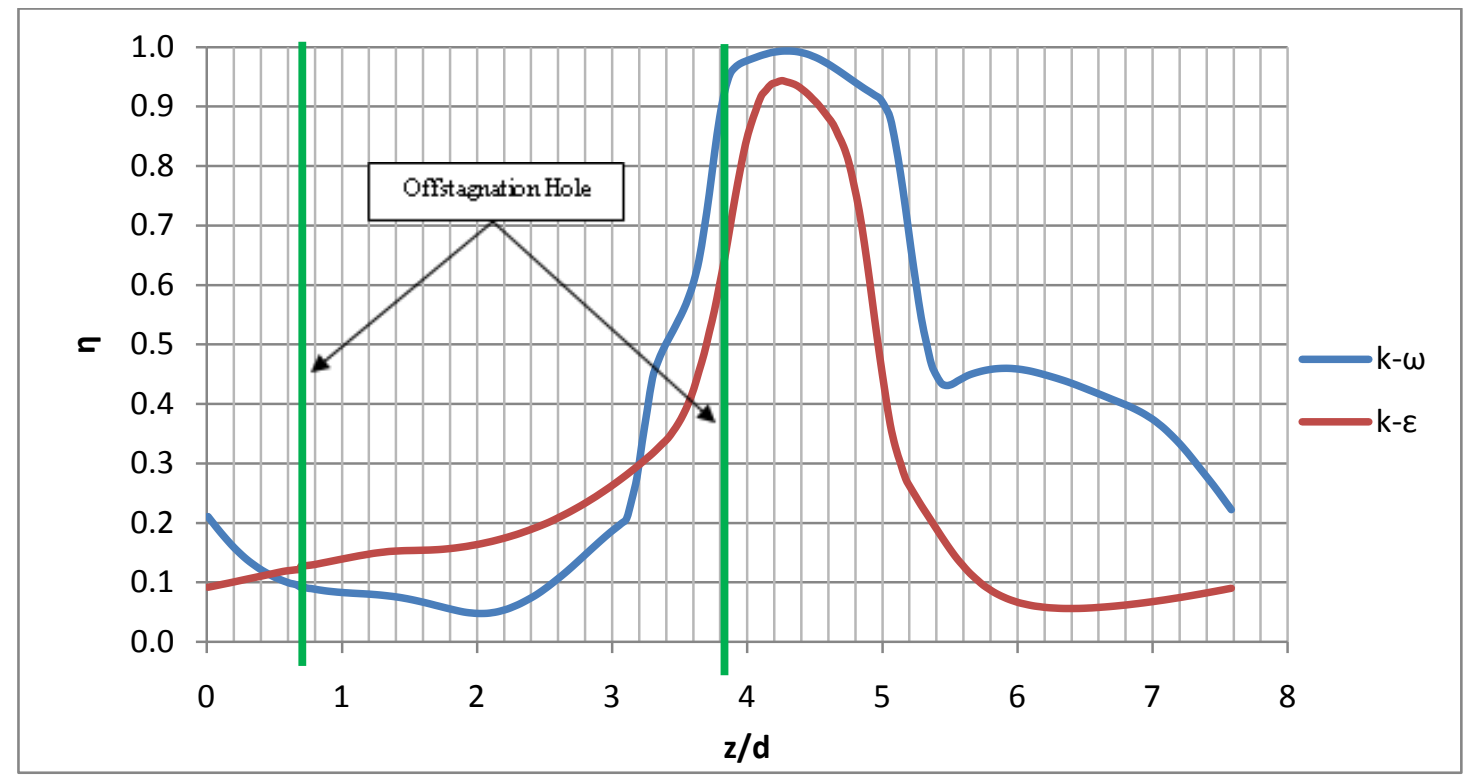

Figure 3.3- Profiles of $\eta$ along $\mathrm{x} / \mathrm{d}=5.1$

The experimental $\eta$ plot is Figure 3.1 also shows an arrow indicating the region of highest $\eta$ when moving downstream of the stagnation row. At the stagnation line, the coolant turns downstream at a distance from the exit hole intermediate between the k- $\varepsilon$ and the k- $\omega$. Stagnation and offstagnation coolant interaction is similar to the $\mathrm{k}-\omega$, with the stagnation coolant interacting with the offstagnation coolant a further distance from the offstagnation exit hole than the k- $\varepsilon$. In the region downstream of the offstagnation row the experimental coolant covers over a greater span length than predicted by k- $\varepsilon$. But even though the experimental coolant flow path agrees better with the k- $\omega$ simulation, the k- $\omega$ laterally average $\eta$ values are consistently higher than the experimental values for most of the leading edge. Also, the k- $\varepsilon$ simulations predictions of laterally average $\eta$ agree very closely with the experiments in the regions between cooling holes rows and regions downstream of the offstagnation row of holes. 


\subsection{Analysis of Thermal Field in the Stagnation Plane}

This section analyses the stagnation plane $(\mathrm{x} / \mathrm{d}=0)$ of the leading edge model. Thermal profiles of the stagnation plane will be compared along with predicted turbulent viscosity and turbulence levels.

The CFD simulations were conducted on both adiabatic and conducting models. While adiabatic models better show coolant flow over the surface, the off the surface temperature profiles are similar for both models. Figure 3.4 is used for comparison of the conducting and adiabatic model profiles at $\mathrm{z} / \mathrm{d}=2.5$. The flow of coolant through the coolant hole results in increase of the coolant exit temperature for the conducting model while it remains $200 \mathrm{~K}$ for the adiabatic model. In order for better comparison in Figure 3.4 , the temperature profile for conducting model is normalized used a modified $\theta$ defined as

$$
\theta^{\prime}=\frac{T_{\text {gas }}-T_{\infty}}{T_{\text {coolant }, \text { exit }}-T_{\infty}}
$$

Where $T_{\text {coolant,exit }}$ is the average temperature of coolant at exit hole. Figure 3.4 reveals that the thermal profiles for conducting $\theta^{\prime}$ and adiabatic $\theta$ are very similar. Since the adiabatic and conducting thermal profiles are similar if scaled properly, thermal profile of the conducting can be used to describe coolant flow over an adiabatic model. The following sections will use the conducting model for analysis. 


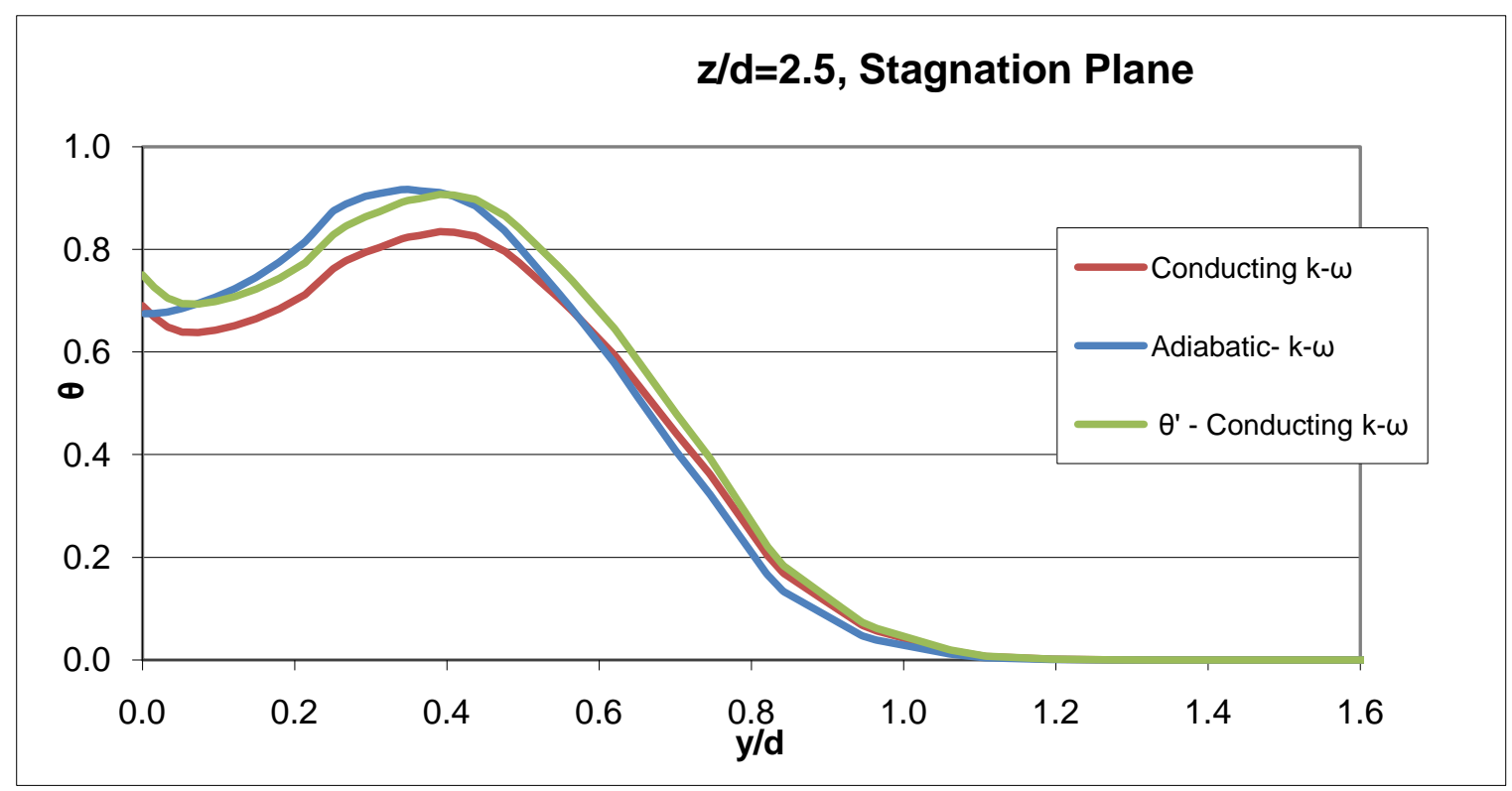

Figure 3.4- Temperature profile at $\mathrm{z} / \mathrm{d}=2.5$ and $\mathrm{x} / \mathrm{d}=0$

\subsubsection{Velocity at the Coolant Exit}

Figure 3.1 showed the coolant flow field upon exiting the stagnation hole is different for the two turbulence models. The simulations were run on leading edge models of similar cooling hole geometry and the mass flow rate at the impingement plate inlet hole is also the same for both simulations. Thus the mass flow rate and velocity profiles of the two models were checked at the stagnation exit hole in order to explain the differences in coolant flow.

For $\mathrm{M}=2$ and $\mathrm{DR}=1.5$ and the three row leading edge model, the $\mathrm{k}-\varepsilon$ stagnation hole had $26.2 \%$ of the total mass flow rate while the k- $\omega$ stagnation hole had $24.8 \%$ of the total mass flow rate. The mass flow rate through the stagnation exit hole is $5.2 \%$ 
higher for the k- $\varepsilon$ compared to $k-\omega$. Thus, differences in coolant flow within the model interior results in slightly more coolant exiting the stagnation hole for the $\mathrm{k}-\varepsilon$ simulation.

Figure 3.5 represents the velocity vectors at the exit of the stagnation hole. It is evident that the $k-\omega$ coolant separates from the model surface upon exiting the stagnation

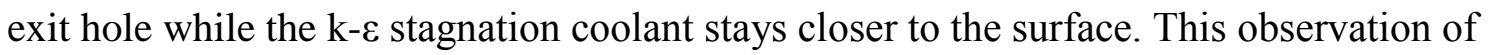
coolant behavior agrees with Figure 3.1.

Analysis of the velocity profile along the stagnation line of the coolant hole shows that the average velocity magnitude is $15 \%$ higher for the $\mathrm{k}-\varepsilon$ model. The average velocity in the spanwise direction is $13.5 \%$ higher for the $\mathrm{k}-\varepsilon$ and the average velocity in the normal direction is $10 \%$ higher for the $\mathrm{k}-\varepsilon$. This is consistent with higher coolant mass flow rate through the stagnation hole for the k- $\varepsilon$. For the stagnation line, the k- $\omega$ stagnation coolant exits the hole at an average angle of $19.2^{\circ}$ with respect to the model surface while the k- $\varepsilon$ forms an average angle of $18.7^{\circ}$. Thus while the average velocity magnitude at the stagnation line differ by $10 \%$ between the turbulence models, they have similar direction of exit. 


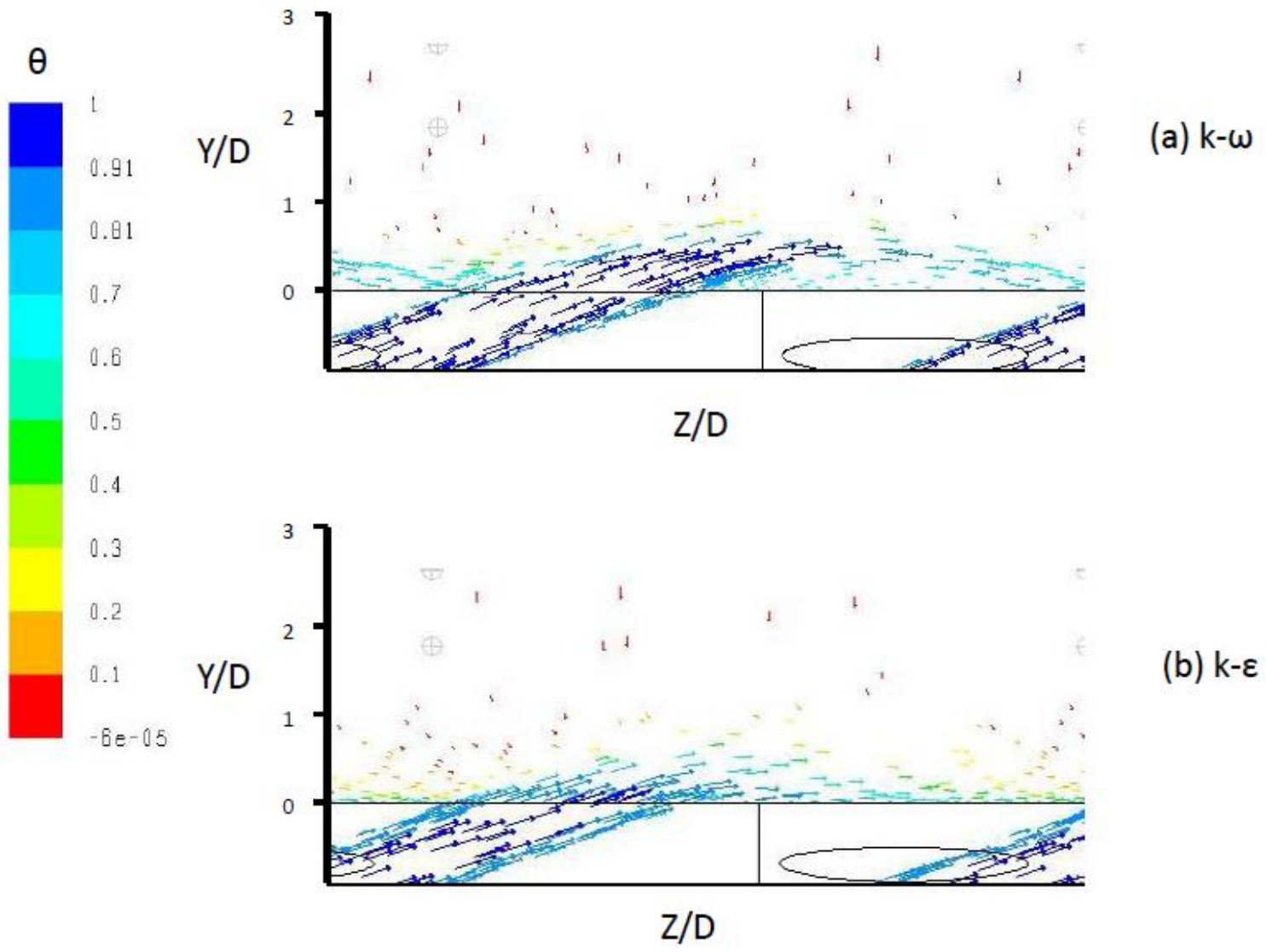

Figure 3.5- Velocity Vector colored by $\theta$, along stagnation plane 


\subsubsection{Separation along the Stagnation Plane}

As seen in the surface contours plots, there are differences between the two turbulence models along the stagnation region. This difference in the surface temperatures can be attributed to the models predicting different coolant flow paths after it exits the stagnation hole. Thermal field measurements are compared along the stagnation plane and the temperatures are expressed in terms of non dimensional gas temperature $\theta$. The experimental thermal field data was collected using the thermocouple probe described earlier. Individual off the wall profiles were taken at spanwise intervals of $1.59 \mathrm{~mm}$. For each plane, 16 such measurements were made to complete one pitch distance. Deltagraph software was used to create a contour plot from the individual profiles. The test conditions of $\mathrm{M}=2$ and $\mathrm{DR}=1.5$ maintained for the measurements.

Figure 3.6 compares the experimental thermal field measurements at the stagnation plane. The stagnation coolant hole location is also shown in the Figure 3.6. As stated in earlier section, the k- $\omega$ profile shows separation of the coolant from the model surface while the k- $\varepsilon$ coolant stays attached throughout the span length. Examination of the experimental profile reveals that the coolant experiences separation at the stagnation plane. For the experimental data, separated coolant behavior can be seen extending up to $\mathrm{z} / \mathrm{d}=1.9$ from the lip of the stagnation hole. The coolant mixing and the slopes of the temperature profiles also show differences between contour plots in Figure 3.6. Individual profiles will be compared for better assessment of the stagnation coolant behavior in the stagnation plane 


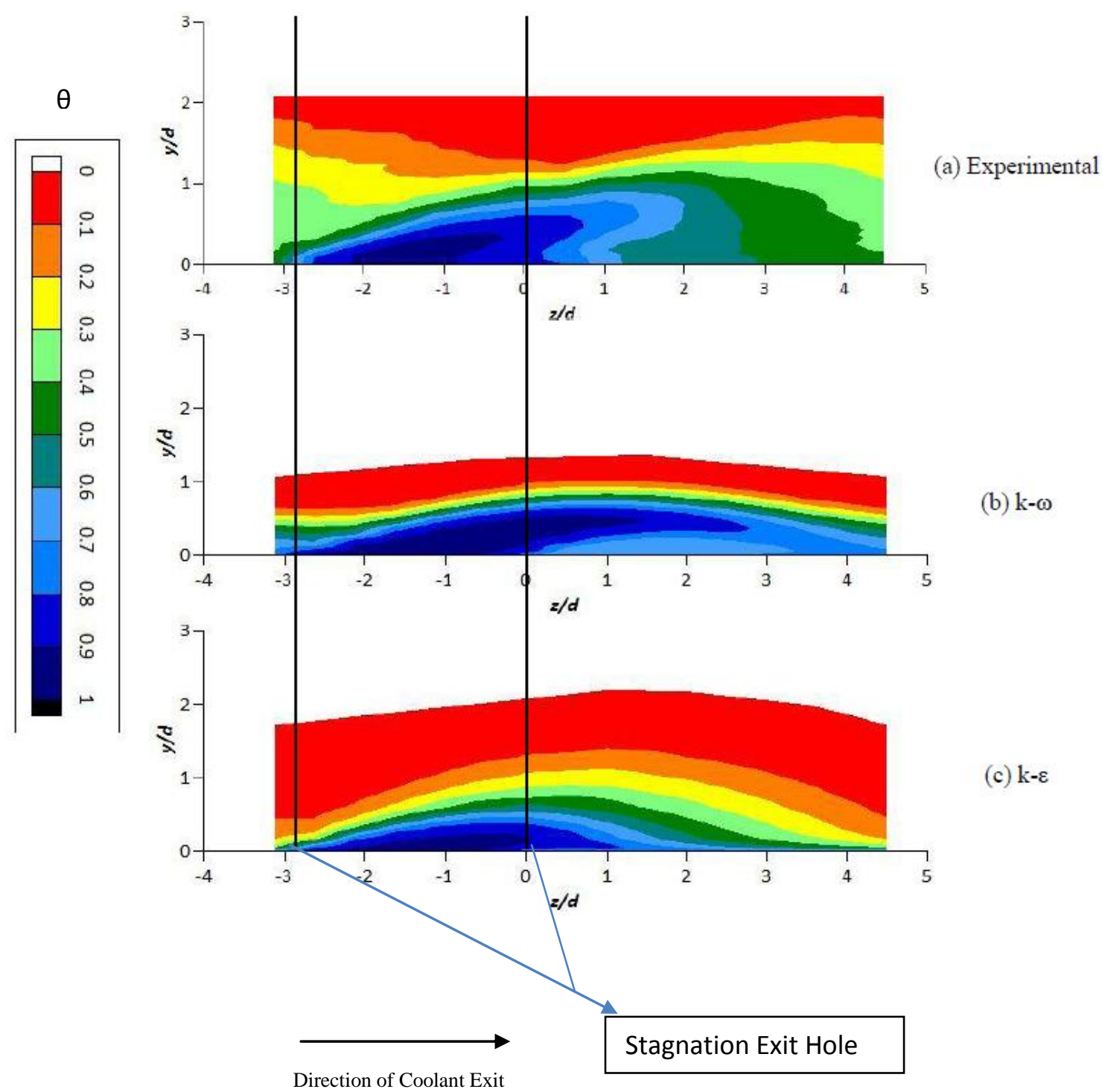

Figure 3.6- Stagnation Plane thermal profile- (a) Experimental, (b) k- $\omega$ and (c) k- $\varepsilon$ 
Figure 3.7 can be used to demonstrate the extent of coolant separation for the experimental data. At $\mathrm{z} / \mathrm{d}=1$, it can be clearly seen that location of highest $\theta$ lies at $\mathrm{y} / \mathrm{d}=$ 0.58 from the surface. For $\mathrm{z} / \mathrm{d}=2$, the location of highest $\theta$ also lies further away from the surface at $y / d=0.67$. Thus, both locations show signs of coolant separation. The core of the coolant jet is colder at $\mathrm{z} / \mathrm{d}=1$ and it lies closer to the surface. Upon travelling further in the spanwise direction to $\mathrm{z} / \mathrm{d}=2$, the coolant jet undergoes increased mixing. At this further span location, the coolant is warmer and is more uniformly distributed off the model surface.

Figure 3.7 also compares the experimental data with the computational profiles. At $\mathrm{z} / \mathrm{d}=1$, the $\mathrm{k}-\omega$ simulation captures coolant separation. While the core of the coolant jet lies at similar distances off the wall for the k- $\omega$ and experiment, the $\mathrm{k}-\omega$ has a colder coolant jet and thus shows considerably less mixing with the mainstream. The k- $\varepsilon$ profiles do not show coolant separation at either locations and also over predicts the surface $\theta$. 


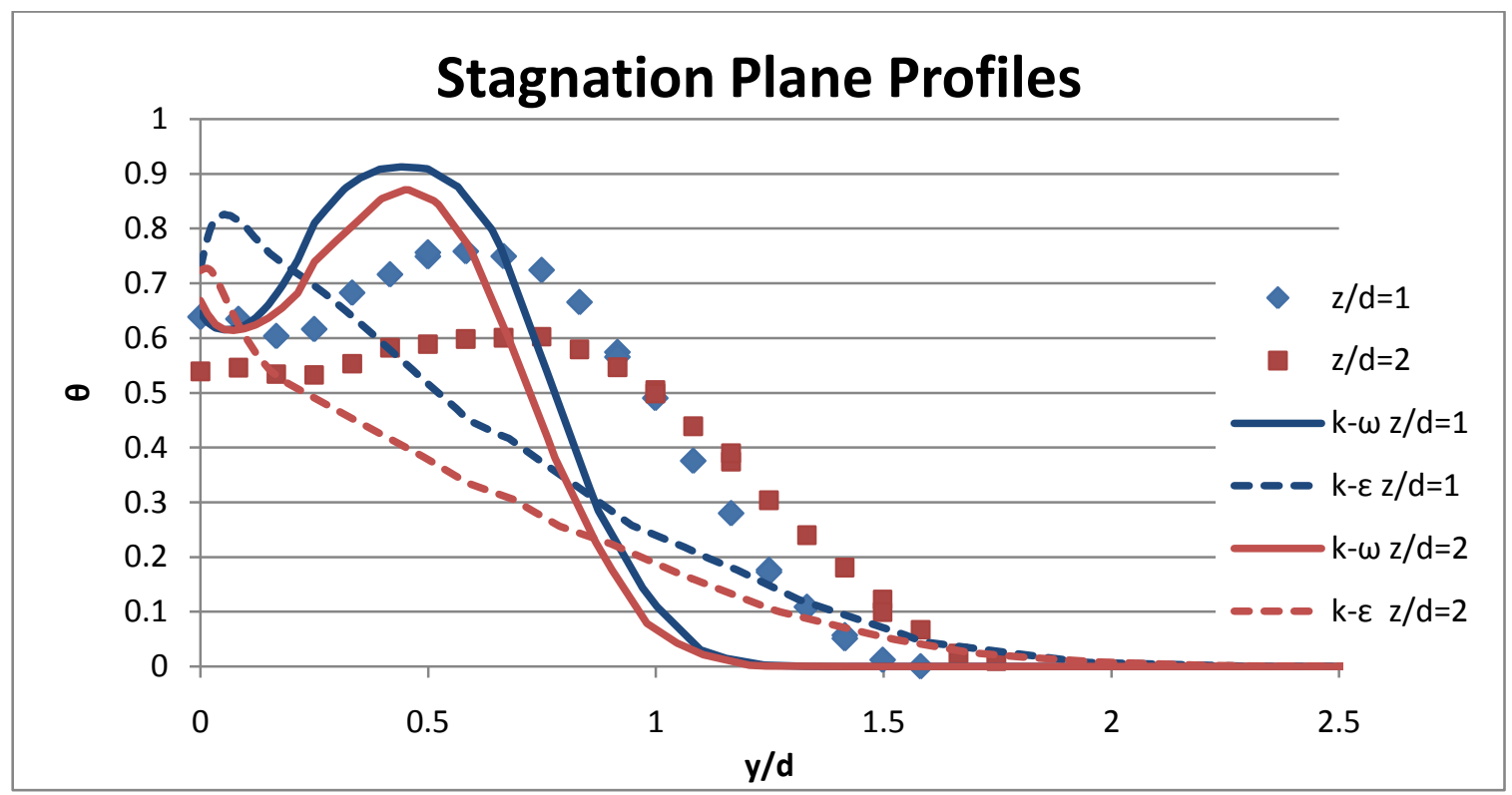

Figure 3.7- Off the wall profiles at the stagnation plane

\subsubsection{Mixing of the Coolant with Mainstream}

The interaction of the coolant with the mainstream results in diffusion of the colder coolant into the hotter mainstream gas. The contour plots in Figure 3.6 show significant differences in the extent of coolant diffusion into the mainstream between the CFD models and the experiment. The variation of the diffusion length in the spanwise direction is also different between the two models. The $\mathrm{k}-\varepsilon$ prediction of stagnation coolant flow in the stagnation plane extends further away from the surface compared to the $\mathrm{k}-\omega$ simulations.

The diffusion of the coolant can be compared using the $\theta=0.1$ isotherm distance. Figure 3.8 compares the distance from the surface to the point of $\theta=0.1$, where the gas 
temperature is $90 \%$ of the mainstream temperature. It is clear that the experimental data shows coolant extending further into the mainstream than both CFD simulations. The pattern of the 0.1 isotherm growth is also different. The CFD simulations show similar patterns with a decrease in thickness from $\mathrm{z} / \mathrm{d}=1$ until the location of the adjacent stagnation coolant exit hole. The decrease in mixing layer thickness at $\mathrm{z} / \mathrm{d}=1$ could be an indicator of the turning of the stagnation coolant in the downstream direction in the CFD predictions. Thus while the CFD models show different stagnation coolant flowfields, the diffusion of coolant into the mainstream show similar growth in the spanwise direction. The experimental data show the coolant extending further into the mainstream upon travelling in the spanwise direction. The growth in $\theta=0.1$ isotherm thickness continues up until the adjacent exit hole. Thus, the experimental data reveals that for $\mathrm{p} / \mathrm{d}=7.6$, there is significant interaction between coolant jets within the stagnation row.

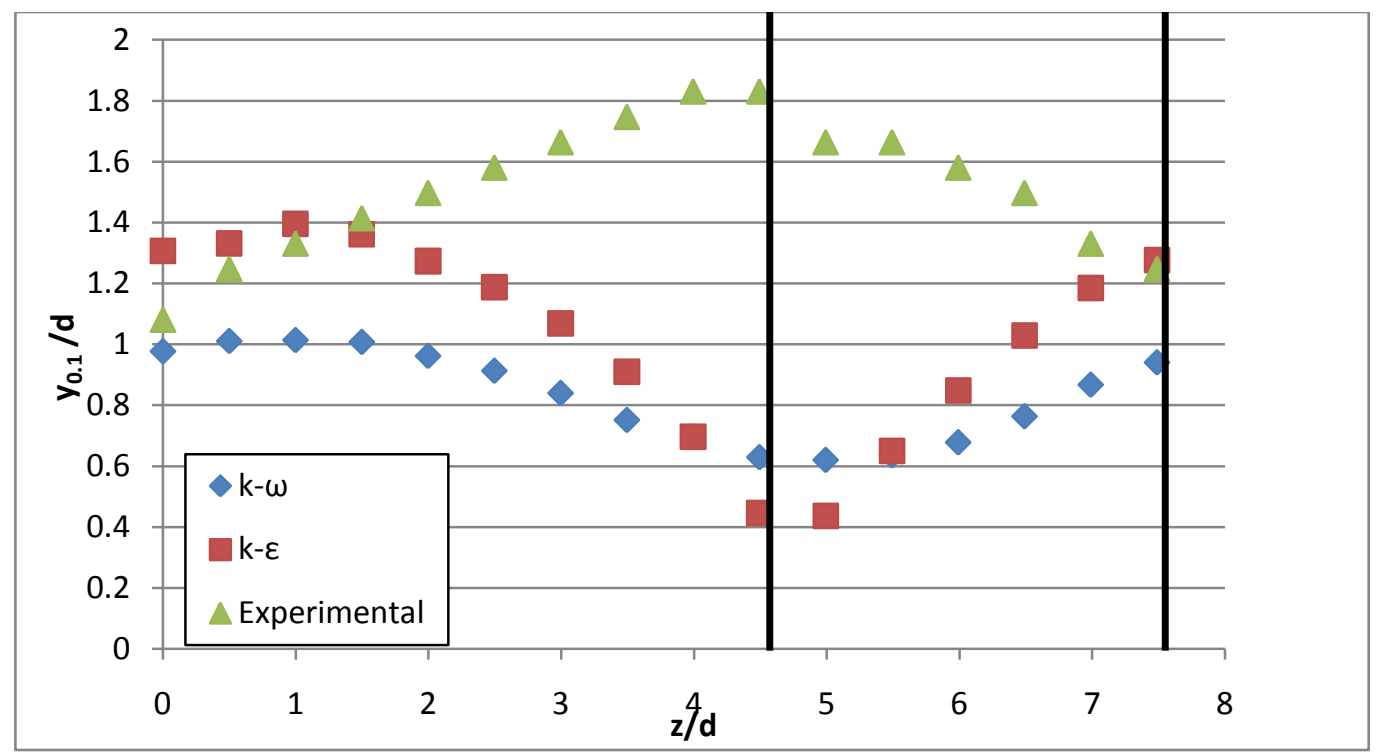

Figure 3.8- Diffusion length thickness along the spanwise direction for $\mathrm{x} / \mathrm{d}=0$ 


\subsubsection{Temperature Profiles in the Stagnation Plane}

Temperature profiles will be examined at different span locations along the stagnation plane in order to better compare the computational and experimental data. Profiles at $\mathrm{z} / \mathrm{d}=$ 0,2 , and 4 will be analyzed.

Figure 3.9 compares the $\theta$ profiles at the lip of the stagnation coolant hole. At this span, the experimental data compares equally well both CFD simulations. Both computational models correctly predict the magnitude and location of the peak $\theta$ values. The general slope of the temperature profile is compared for sections of the profile that

form a straight length. The experimental slope of $\frac{\mathrm{d} \theta}{d(y / d)}=0.82$ lies closer to the $\mathrm{k}-\varepsilon$ slope of 0.76 than the $k-\omega$ value of 1.71 . But the experimental $\theta=0.1$ isotherm location is similar to the $\mathrm{k}-\omega$. Thus, at the $\mathrm{x} / \mathrm{d}=0$, the both simulations agrees equally well with the experimental profile, with the k- $\varepsilon$ profile showing closer slope values. 


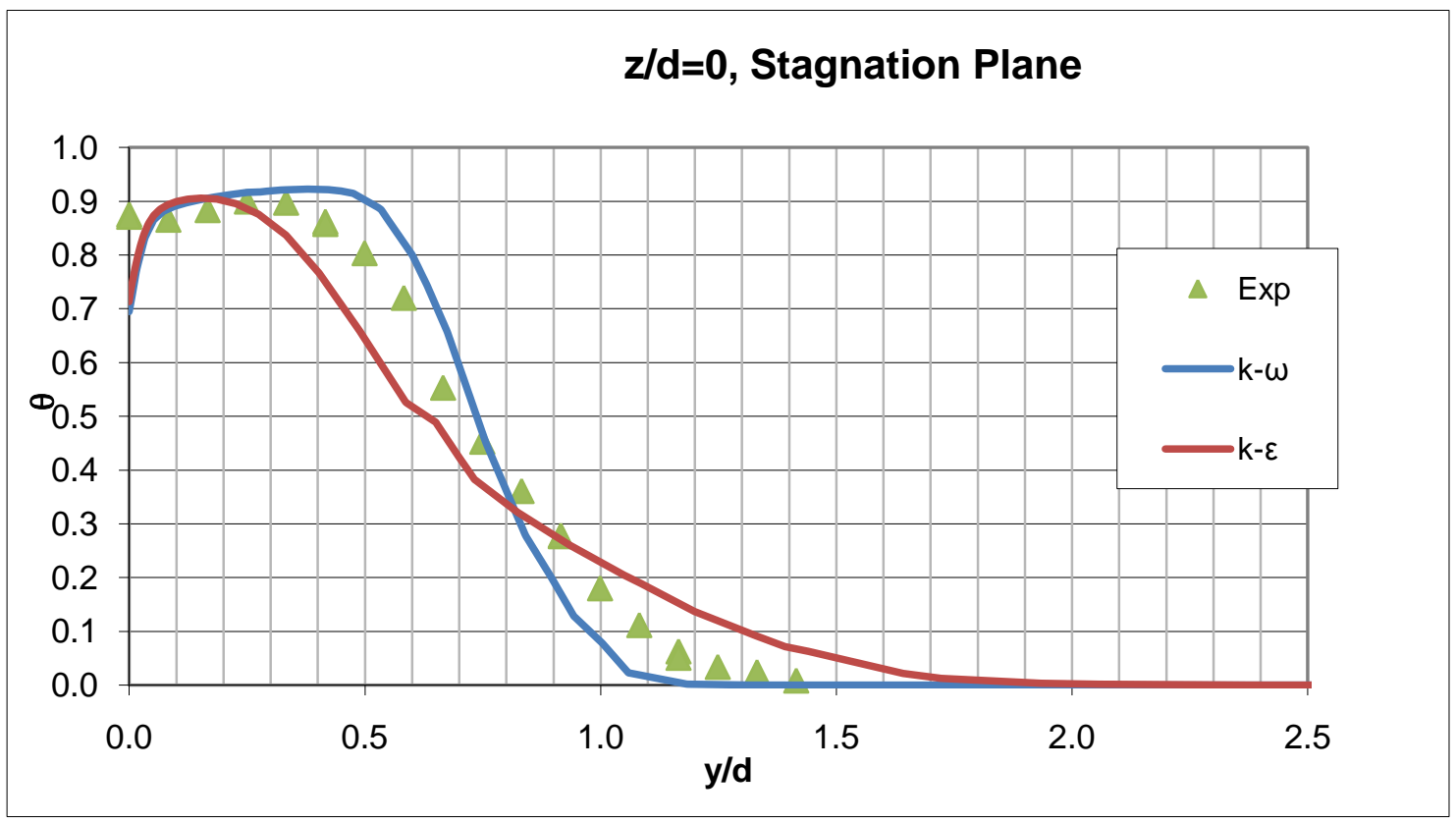

Figure 3.9- Temperature Profile at $\mathrm{z} / \mathrm{d}=0$ along the stagnation plane

At the $\mathrm{z} / \mathrm{d}=2$ span location, the experimental data show significant variation from both simulations (Figure 3.10). Besides the separation issues that were discussed in the earlier section, the experimental data follows a slope different from both simulations. For $\mathrm{k}-\omega$, the slope of the $\theta$ profile is 1.57 while the $\mathrm{k}-\varepsilon$ has a much lower slope of 0.41 . The experimental data shows an almost constant temperature up until $y / d=0.7$ from the surface and then continue to decrease in $\theta$ with a slope of 0.61 . While the shapes of the k$\varepsilon$ and experimental profiles differ, the experimental slope value is similar to the $\mathrm{k}-\varepsilon$ profile and the coolant layer extends to similar distances off the surface for the experimental and $\mathrm{k}-\varepsilon$ simulation. 


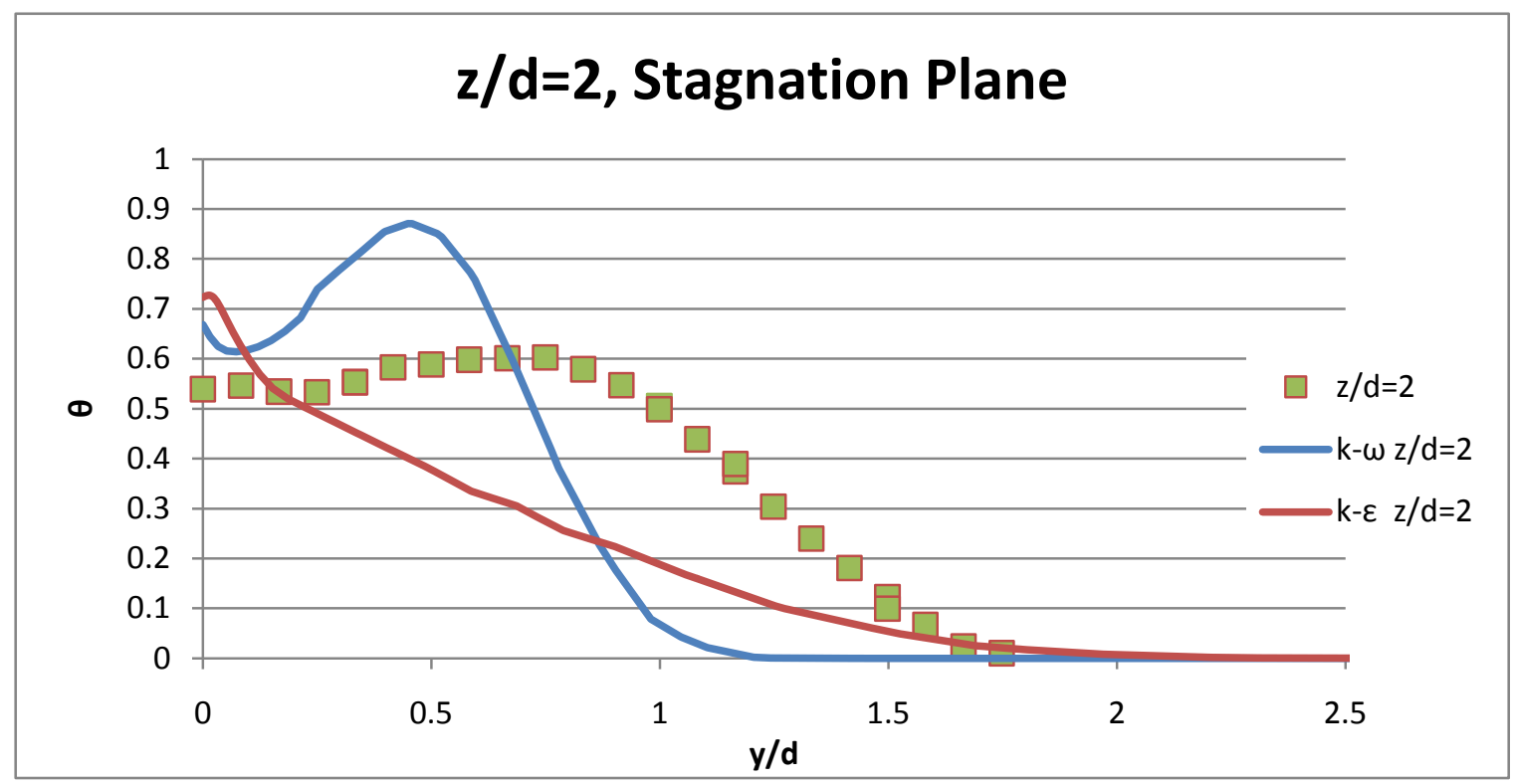

Figure 3.10- Temperature Profile at $\mathrm{z} / \mathrm{d}=2$ along the stagnation plane

Figure 3.10 also reveals that the peak values of $\theta$ are higher for the computational models. The experimental peak $\theta$ value of 0.6 is $21 \%$ and $43 \%$ lower than $\mathrm{k}-\varepsilon$ and $\mathrm{k}-\omega$ respectively. The warmer coolant for the experimental model can be a result of the increased mixing of the coolant relative to the CFD simulation and a difference in the amount of coolant turning downstream of the stagnation line.

Upon moving further in the span direction to $\mathrm{z} / \mathrm{d}=4$, Figure 3.11 also shows differences in $\theta$ profiles. Similar to the experimental profile at $\mathrm{z} / \mathrm{d}=2$, the temperature remains relatively constant up to $y / d=0.6$ from the surface and then decreases with a slope of 0.27 . Meanwhile, both computational models show peak $\theta$ value at the surface. All three profiles in Figure 3.11 show different variation. The k- $\varepsilon$ profile has a steep 
decrease in $\theta$ value close to the surface and then decreases with a low slope of .18. Such a profile is indicative of low coolant presence. The k- $\omega$ profile decreases with a much higher slope of 1.24 and shows coolant presence at this span location.

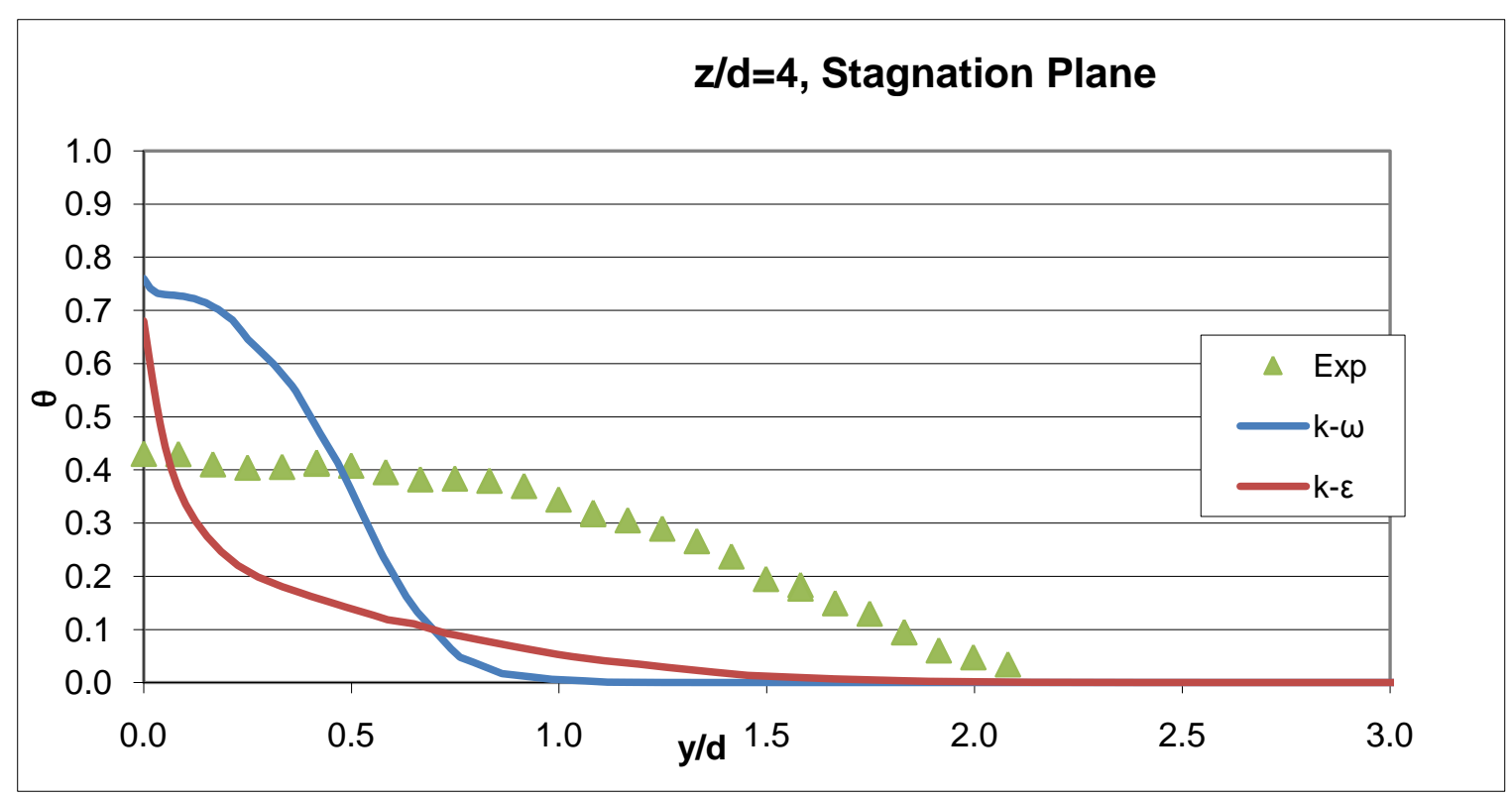

Figure 3.11- Temperature Profile at $\mathrm{z} / \mathrm{d}=4$ along the stagnation plane

Like the profiles at $\mathrm{z} / \mathrm{d}=2$, the experimental peak values of $\theta$ is significantly less. Diffusion is also different, with the coolant extending significantly further into the mainstream for the experimental data. Thus at $\mathrm{z} / \mathrm{d}=4$, the experimental data differs from the simulations in peak values, temperature slopes and coolant diffusion.

\subsubsection{Turbulence Characteristics}

Modeling of turbulent flows requires solving for the Reynolds shear stress. The Reynolds shear stress defines the interaction of the turbulent eddies with flow and thus 
controls the mixing of the mainstream with the coolant. Both k- $\omega$ and k- $\varepsilon$ turbulence models estimate the Reynolds shear stress using the turbulent viscosity hypothesis. The turbulent viscosity hypothesis models the Reynolds shear stress $\left\langle\mathrm{u}_{\mathrm{i}} \mathrm{u}_{\mathrm{j}}\right\rangle$ as

$$
<\mathrm{u}_{\mathrm{i}} \mathrm{u}_{\mathrm{j}}>=-\frac{\mu_{\mathrm{T}}}{\rho}\left(\frac{\delta<\mathrm{U}_{\mathrm{i}}>}{\delta \mathrm{x}_{\mathrm{j}}}+\frac{\delta<\mathrm{U}_{\mathrm{j}}>}{\delta \mathrm{x}_{\mathrm{i}}}\right)
$$

Where $\mu_{\mathrm{T}}$ is the turbulent viscosity and $\frac{\delta<\mathrm{U}_{\mathrm{i}}>}{\delta \mathrm{x}_{\mathrm{j}}}$ is the gradient of mean velocity. Earlier section showed that the stagnation coolant had similar velocity vector at the exit hole for the two turbulence models. The magnitude and direction of coolant at the exit hole is similar for the two turbulence models even though the coolant had different flow paths when travelling away from the hole.

With the turbulence models having with similar blowing ratios, coolant hole geometry and similar exit velocity profiles, the difference in mixing layer thickness can be attributed to turbulent viscosity. Figure 3.12 shows the turbulent viscosity contours for the models at the stagnation plane for two pitch lengths. The k- $\varepsilon$ simulation shows much higher turbulent viscosity levels than the k- $\omega$. The k- $\varepsilon$ has highest $\mu_{\mathrm{T}}$ levels at $\mathrm{y} / \mathrm{d}=2.35$ from the surface and this distance represent the edge of the mixing layer. Meanwhile, the k- $\omega$ simulation shows much lower values of $\mu_{\mathrm{T}}$, of magnitude less than $0.0055 \mathrm{~kg} / \mathrm{m}-\mathrm{s}$. Figure 3.12 shows the $\mu_{\mathrm{T}}$ profile for the $\mathrm{k}-\omega$ simulation at $\mathrm{z} / \mathrm{d}=0$, it has a value of zero at the wall and continues to increases even up to $y / d=3$ and beyond. With higher turbulent viscosity for $\mathrm{k}-\varepsilon$, the Reynolds shear stress is higher and thus the diffusion of the coolant is greater. The $\mathrm{k}-\varepsilon$ also shows elevated $\mu_{\mathrm{T}}$ even at $\mathrm{y} / \mathrm{d}=4.4$ from the surface. At this 
distance, even though the stagnation effects of the model are felt by the mainstream, there is no coolant presence.

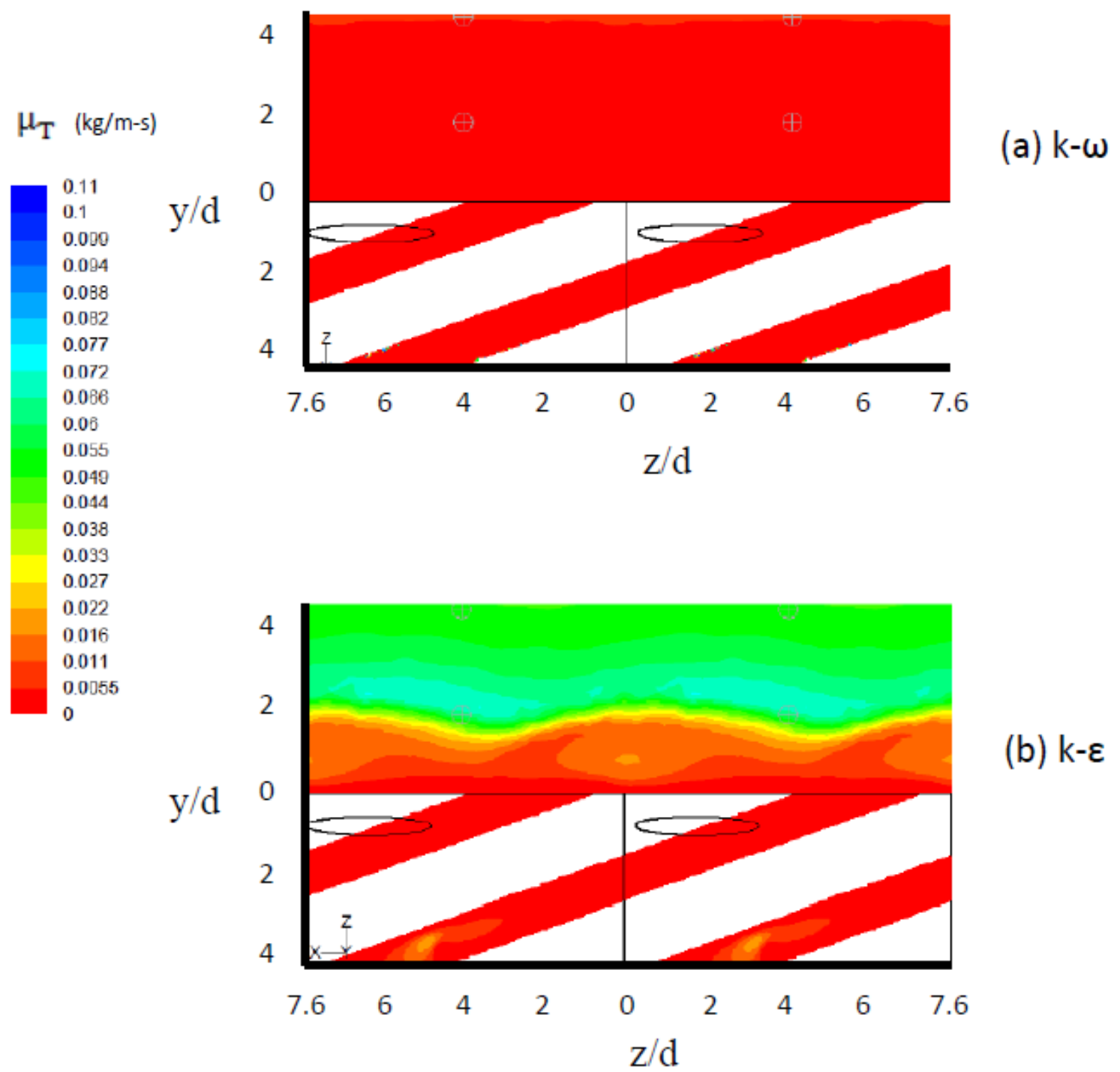

Figure 3.12- Contours of Turbulent Viscosity (kg/m-s) at Stagnation plane

As expected the turbulent energy production goes to zero at the wall. As for regions further away from the surface, the coolant interaction can be seen analogous to shear layers where the coolant propagates into the free stream by diffusion. In simpler 2-D 
flows, Pope (2000) indicates that the standard k- $\omega$ model has problems in predicting fluid mixing as the freestream is approached. The SST model for k- $\omega$ solves this problem of free shear behavior by using a blending function to combine the ability of k- $\omega$ in the near wall region with the ability of k- $\varepsilon$ to better predict free shear layers. The blending function is designed to be near unity or k- $\omega$ in the inner half of the wall boundary layer and then decrease until zero or k- $\varepsilon$ at the top edge. Hence the function is dependent on distance away from the wall.

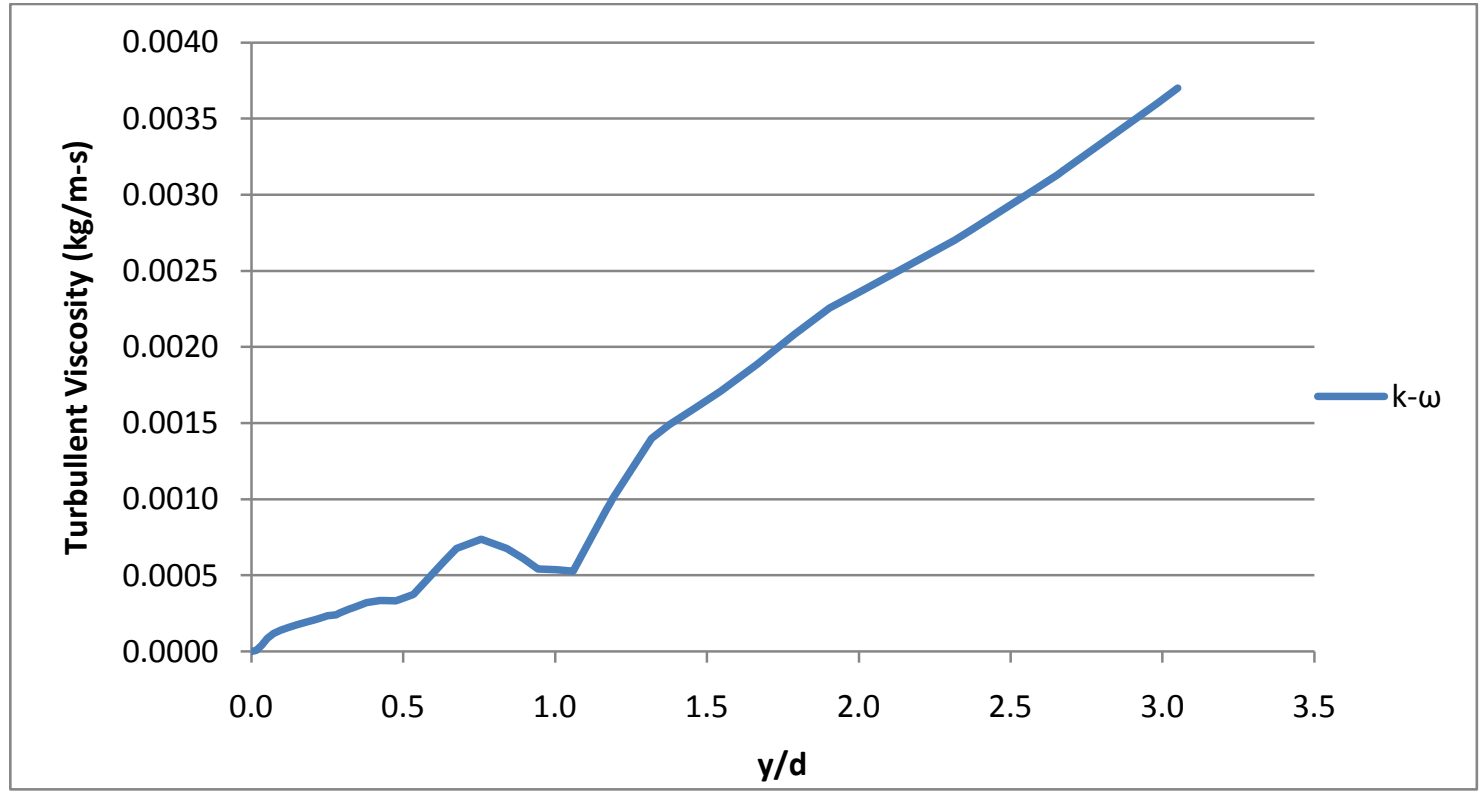

Figure 3.13 - Turbulent Viscosity Profile at $\mathrm{z} / \mathrm{d}=0$ and along stagnation plane

In the current simulations, the $\mathrm{k}-\omega$ coolant does predict coolant separation and hence shows differences from the $\mathrm{k}-\varepsilon$ model close to the wall. But further away from the surface, in the free stream edge region, turbulence viscosity contours of Figure 3.12 shows that the turbulence models behave differently. It is possible the transition of the k- 
$\omega$ to k- $\varepsilon$ as per the SST k- $\omega$ model does not occur correctly for the complex film cooling flow. Thus in the mixing regions of the coolant and mainstream, the k- $\omega$ model continues to exist instead of $k-\varepsilon$ and this could result in unreliable coolant mixing predicting. Kovzulovic and Rober (2006) mentions that the SST k- $\omega$ model with wall distance based bending function can result in erroneous predictions for complex 3-D flows such as in turbomachinery. It was proposed that a blending function that accounted for curvature influence and was calculated based only on physical characteristics of the flow and not based on wall distance, would provide more accurate transition between the k- $\omega$ and the k- $\varepsilon$ models.

The freestream velocity had an inlet condition of $6 \%$ turbulence intensity. With coolant interaction and the decrease in men velocity as the wall is approached, turbulence intensity will increase closer to the model. The turbulence intensity (I) for this study was defined as the

$$
I=\frac{\sqrt[2]{\frac{2 * k}{3}}}{U_{\text {local }}}
$$

where $\mathrm{U}_{\text {local }}$ is the mean velocity magnitude at that location.

Even though freestream velocity is $15 \mathrm{~m} / \mathrm{s}$, the local velocity magnitude along the stagnation plane can decrease to $0 \mathrm{~m} / \mathrm{s}$ at the wall and results in the turbulence intensity levels being excess of $100 \%$. Thus for the purposes of comparison, the modified quantity called turbulence level (I') is used where 


$$
I^{\prime}=\frac{\sqrt[2]{\frac{2 * k}{3}}}{U_{\text {approach }}}
$$

Where $U_{\text {approach }}$ is the of the approach flow $(15 \mathrm{~m} / \mathrm{s})$.

Figure 3.14 represents the contour plots of turbulence level along the stagnation plane. The k- $\varepsilon$ model shows higher percent turbulence intensity between $38 \%$ and $32 \%$ while the highest turbulence level is lower for $\mathrm{k}-\omega$, between $25 \%$ and $19 \%$. The $\mathrm{k}-\varepsilon$ simulation experiences higher turbulence levels over a greater area and for greater distances away from the surface. This region higher turbulence levels correspond to region of coolant mixing with the mainstream. Also with a constant $\mathrm{U}_{\mathrm{avg}}$, increased turbulence intensities translate to higher turbulent kinetic energy for the flow at that location.

The k- $\varepsilon$ simulations were also conducted with $1 \%$ freestream turbulence and these results are shown in Appendix A. 


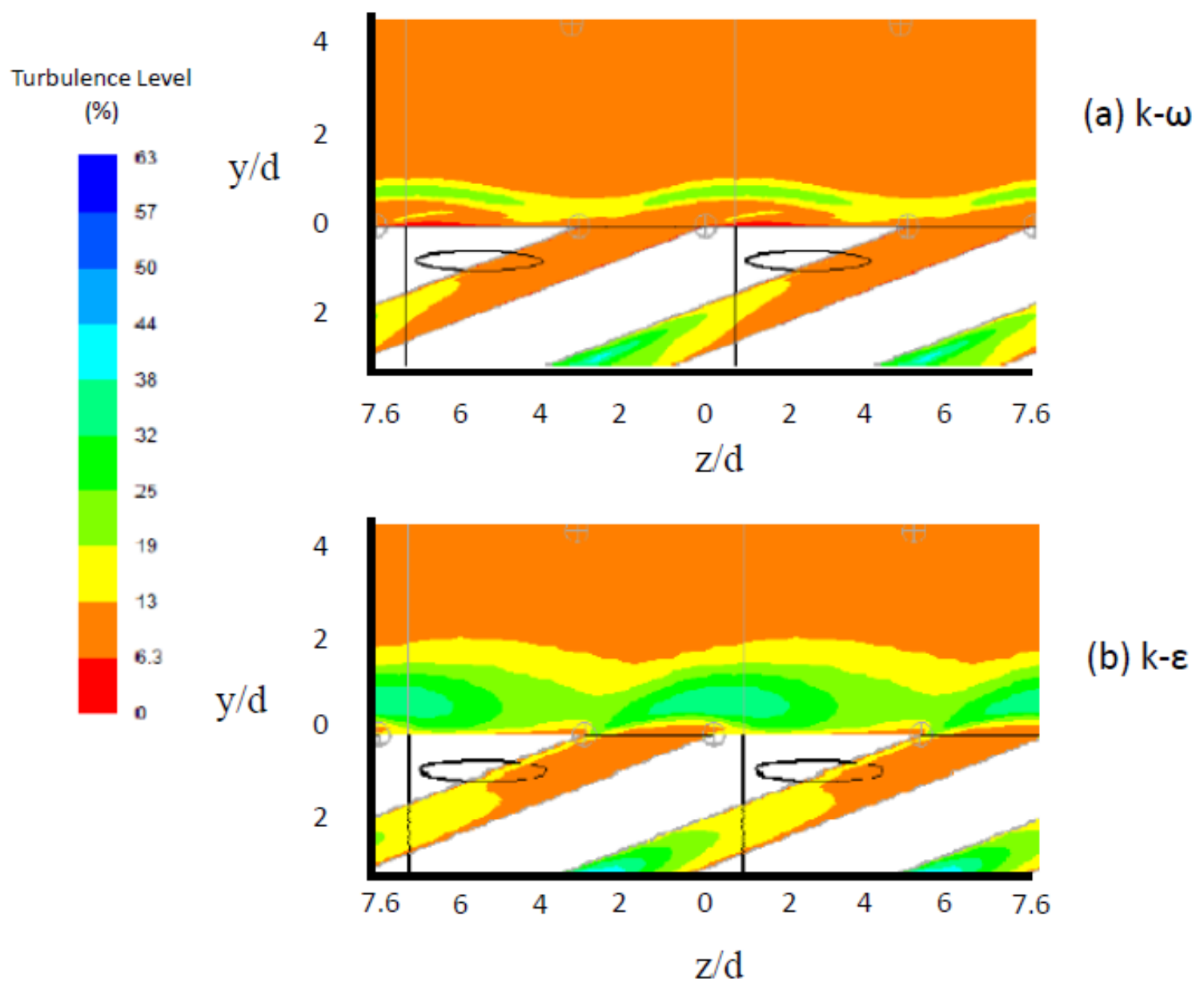

Figure 3.14- Turbulence level contours at stagnation plane

\section{3 - Between the Stagnation and Off Stagnation Row of Holes}

It was shown earlier that the lateral average value of $\eta$ was higher in most regions for the $\mathrm{k}-\omega$. This is also true in the region between the stagnation and off stagnation row of holes. Analysis of coolant exiting the stagnation row had shown that the k- $\varepsilon$ coolant experiences greater mixing with the mainstream. The $\mathrm{k}-\varepsilon$ coolant is also more prone to turn in the mainstream direction than the $\mathrm{k}-\omega$ simulations. 
The $\theta$ profile for $\mathrm{x} / \mathrm{d}=2$ is compared in Figure 3.15 . It can be clearly seen that $\mathrm{k}-\omega$ coolant flow is colder at this downstream location than the $k-\varepsilon$. The peak $\theta$ values for $k-\omega$ coolant lies within 0.7 and 0.6 while for $\mathrm{k}-\varepsilon$ the coolant core is warmer with highest $\theta$ between 0.5 and 0.4 . Also, the core of the coolant jet is located at a different span location for the two models. The k- $\omega$ coolant lies further away from the exit of the stagnation hole lip, at $\mathrm{z} / \mathrm{d}=4.2$ compared to the core of the $\mathrm{k}-\varepsilon$ coolant, located at $\mathrm{z} / \mathrm{d}=2$. Thus the k- $\omega$ stagnation coolant turns in the downstream direction farther downstream than the k- $\varepsilon$. The differences in the coolant temperatures jet and lateral spreading of the stagnation coolant was also confirmed by Figure 3.2 where k- $\omega$ shows colder surface temperatures at distances further away from the stagnation plane than the $\mathrm{k}-\varepsilon$.
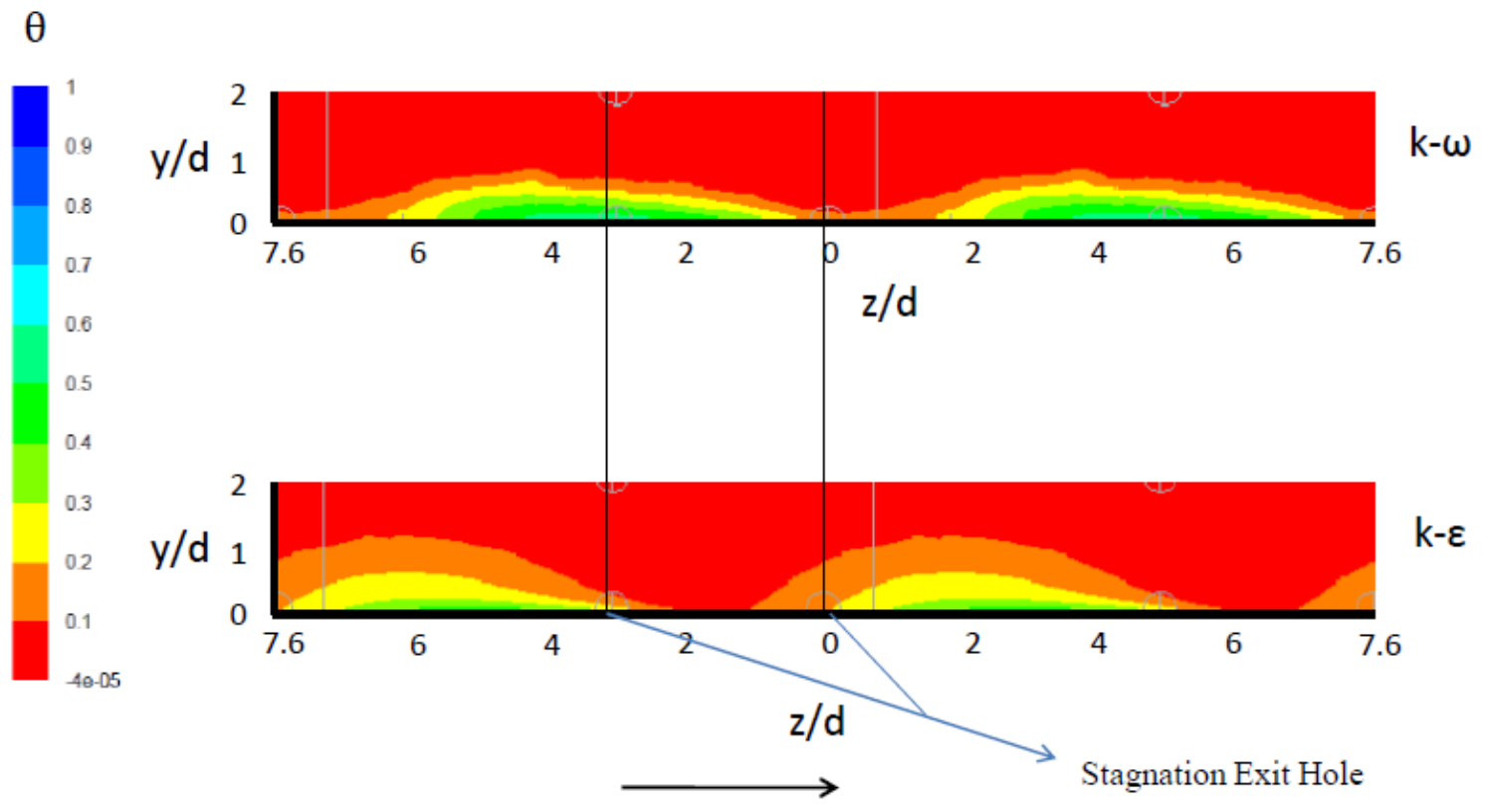

Direction of Coolant

Exit

Figure $3.15-\theta$ contours at $\mathrm{x} / \mathrm{d}=2$ plane 
The mixing between coolant and mainstream also differs at $\mathrm{x} / \mathrm{d}=2$. The $\mathrm{k}-\varepsilon$ shows larger diffusion of coolant into the mainstream. Similar to the stagnation plane, the $\mu_{\mathrm{T}}$ levels are significantly bigger in magnitude for the $\mathrm{k}-\varepsilon$ at $\mathrm{x} / \mathrm{d}=2$. Thus Reynolds shear stress value will be elevated, allowing for greater diffusion of coolant into the mainstream for $\mathrm{k}-\varepsilon$. 


\section{4 - Off Stagnation Plane}

\subsection{1- Thermal Field Measurements}

Along with stagnation plane data, experimental profiles were also measured at $\mathrm{x} / \mathrm{d}=5.1$ for the conducting model .This location allowed for capture of offstagnation coolant behavior and also shows the effects of interaction between coolant from the stagnation line of holes and from the off stagnation holes. The thermal field data is presented for a plane perpendicular to the model surface. The experimental data consists of individual temperature profiles that are spaced $1.59 \mathrm{~mm}$ apart. Figure 3.16 compares the experimental contour plot with the $\mathrm{k}-\omega$ and $\mathrm{k}-\varepsilon$ computational plots at $\mathrm{x} / \mathrm{d}=5.1$. The

temperatures are expressed in terms of non dimensional $\theta$. The location of the offstagnation coolant exit hole is also shown in Figure 3.16.

Figure 3.16 shows that the highest $\theta$ values for the experimental data is between $\theta=$ 0.6 and $\theta=0.7$ while computational models predicted higher values. The k- $\omega$ CFD simulation predicted highest $\theta$ values between 0.9 and 0.8 while the $\mathrm{k}-\varepsilon$ highest $\theta$ values are lower, between 0.8 and 0.7 . With the coldest temperatures representing the core of the offstagnation coolant jet, the experimental values show warmer coolant temperature at this downstream location than the CFD predictions.

It can also be noted that the k- $\omega$ simulation has significantly greater region of colder coolant $(\theta>0.8)$ present at this distance downstream of the offstagnation hole than the k- $\varepsilon$ predictions. Analysis of the plane revealed that the highest theta value is 0.86 for $\mathrm{k}-\omega$ 
while for the $\mathrm{k}-\varepsilon$ is lower at 0.78 . Thus the $\mathrm{k}-\omega$ simulations show less dispersion of the coolant into the mainstream and hence it retains is colder temperature further downstream.

The lateral spreading of the coolant upon exiting the offstagnation hole is also captured by both CFD models. If significant offstagnation coolant presence is defined as regions with $\theta>0.3$, the experimental offstagnation coolant spreads a spanwise distance of $\mathrm{z} / \mathrm{d}=3.3$. The $\mathrm{k}-\omega$ coolant also spreads a similar distance of $\mathrm{z} / \mathrm{d}=3.3$ while the $\mathrm{k}-\varepsilon$ off stagnation coolant is more compact at $\mathrm{z} / \mathrm{d}=2.8$. The general shape of the offstagnation coolant is also similar between contour plots. The region of coldest temperature is attached to the surface for some span length with a significant portion experiencing a hooking over effect in the direction opposite to coolant exit direction. Interaction of the offstagnation coolant with the mainstream results in some coolant being pushed away from the core of the offstagnation coolant jet which remains attached to the wall. 


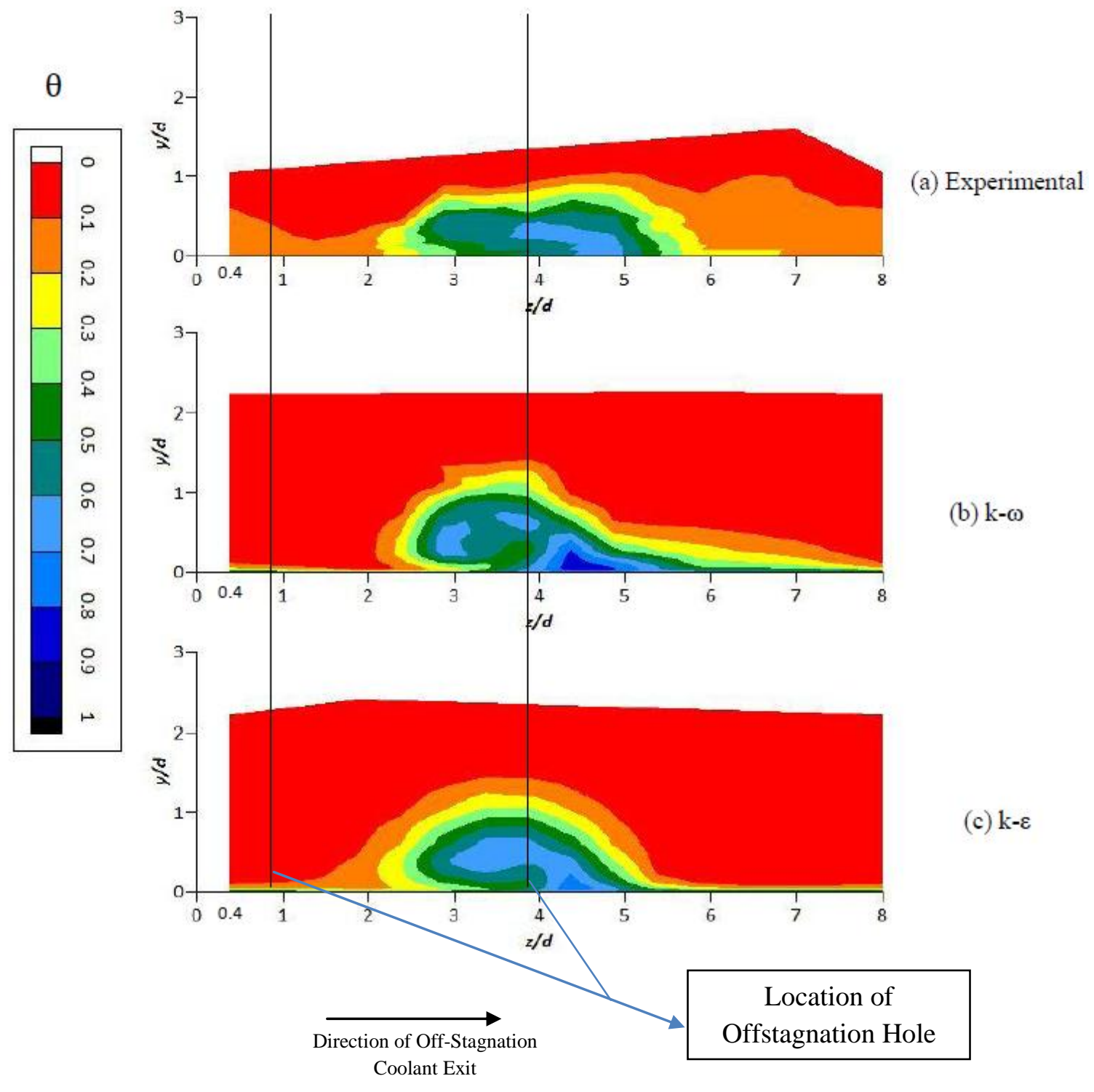

Figure 3.16- Contour plots of $\theta$ at $\mathrm{x} / \mathrm{d}=5.1$ plane 
Figure 3.16 also shows that the thermal fields differ significantly in the region $\mathrm{z} / \mathrm{d}>$ 5.78 with respect to the stagnation exit hole lip. The region of $\theta>0.1$ extends further off the surface for the experimental data compared to the both CFD data. Thus, the experimental data shows the presence of lower temperature gas above the model at this span location. The k- $\omega$ thermal profile also shows stagnation coolant presence for $\mathrm{z} / \mathrm{d}>5.78$ while the $\mathrm{k}-\varepsilon$ shows little coolant presence beyond the offstagnation coolant jet. Since this span location is outside the region of the offstagnation coolant, the higher $\theta$ values can be attributed to the effect of stagnation coolant interacting with the offstagnation row of holes.

In the CFD simulations, the stagnation coolant show different flow fields between simulations. As discussed in earlier section, the k- $\omega$ stagnation coolant turns in the downstream direction at a greater distance from the lip of the stagnation hole than the k- $\varepsilon$ simulation. The k- $\omega$ stagnation coolant interacts with the offstagnation coolant in the $\mathrm{z} / \mathrm{d}>5.78$ region while the $\mathrm{k}-\varepsilon$ stagnation coolant joins the offstagnation coolant in a region closer to the offstagnation exit hole. Since, the experimental and k- $\omega$ data show stagnation coolant presence at similar locations, it can be inferred that the k- $\omega$ model better predicts flow path of the stagnation coolant as it flows downstream.

The experimental data in Figure 3.16 shows coolant extending beyond $y / d=1$ for $z / d$ $>5.78$ while the $\mathrm{k}-\omega$ has coolant extending to lower $\mathrm{y} / \mathrm{d}$ distances of 0.5 . Also, the $\mathrm{k}-\omega$ stagnation coolant present at the $\mathrm{x} / \mathrm{d}=5.1$ location was colder in temperature with peak values of $0.3<\theta<0.4$ while the experimental data has peak values of $0.2<\theta<0.1$ (Figure 
3.16). Thus while the $\mathrm{k}-\omega$ model does predict the stagnation coolant flow path, it under predicts the coolant mixing and diffusion into the mainstream.

\subsubsection{Temperature Profiles}

The temperature profiles will be compared at different span locations in order to better access the differences among the contour thermal fields. Figure 3.17 compares off the surface $\theta$ profiles at the $\mathrm{z} / \mathrm{d}=2.9$ location. This location represents a region of coolant separation from the surface. Figure 3.18 represents the $\theta$ profiles at the $\mathrm{z} / \mathrm{d}=4.9$ and this location represents the core of the offstagnation coolant jet.

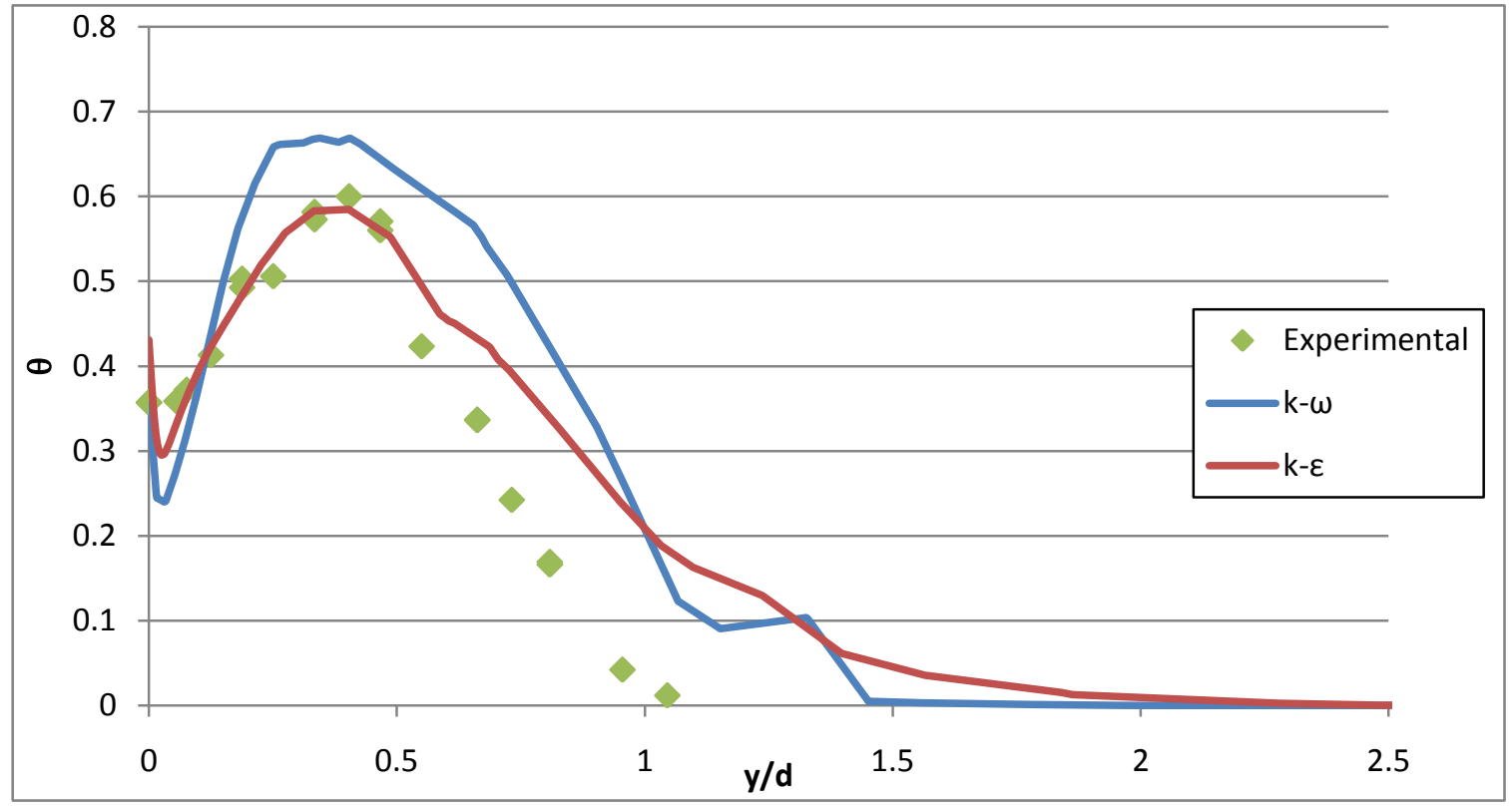

Figure $3.17-\theta$ profile at $\mathrm{z} / \mathrm{d}=2.9$ and $\mathrm{x} / \mathrm{d}=5.1$ 
Figure 3.17 show that the experimental and $k-\varepsilon$ profiles show similar levels of coolant detachment from the surface. The core of the jet is above the surface because of the lateral spreading of the offstagnation jet in the direction opposite to coolant exit due to interaction with the mainstream. The peak $\theta$ value is the same for these profile and they both occur at $\mathrm{y} / \mathrm{d}=0.4$ from the surface. The $\mathrm{k}-\omega$ coolant also shows separation and the peak value occurs at a similar distance off the wall compared to the other profiles but the peak value is higher in magnitude. While the slope of the experimental profile matches the k- $\omega$ profiles better, both CFD simulations show coolant extending to further distance off the surface compared to the experimental data at this span location.

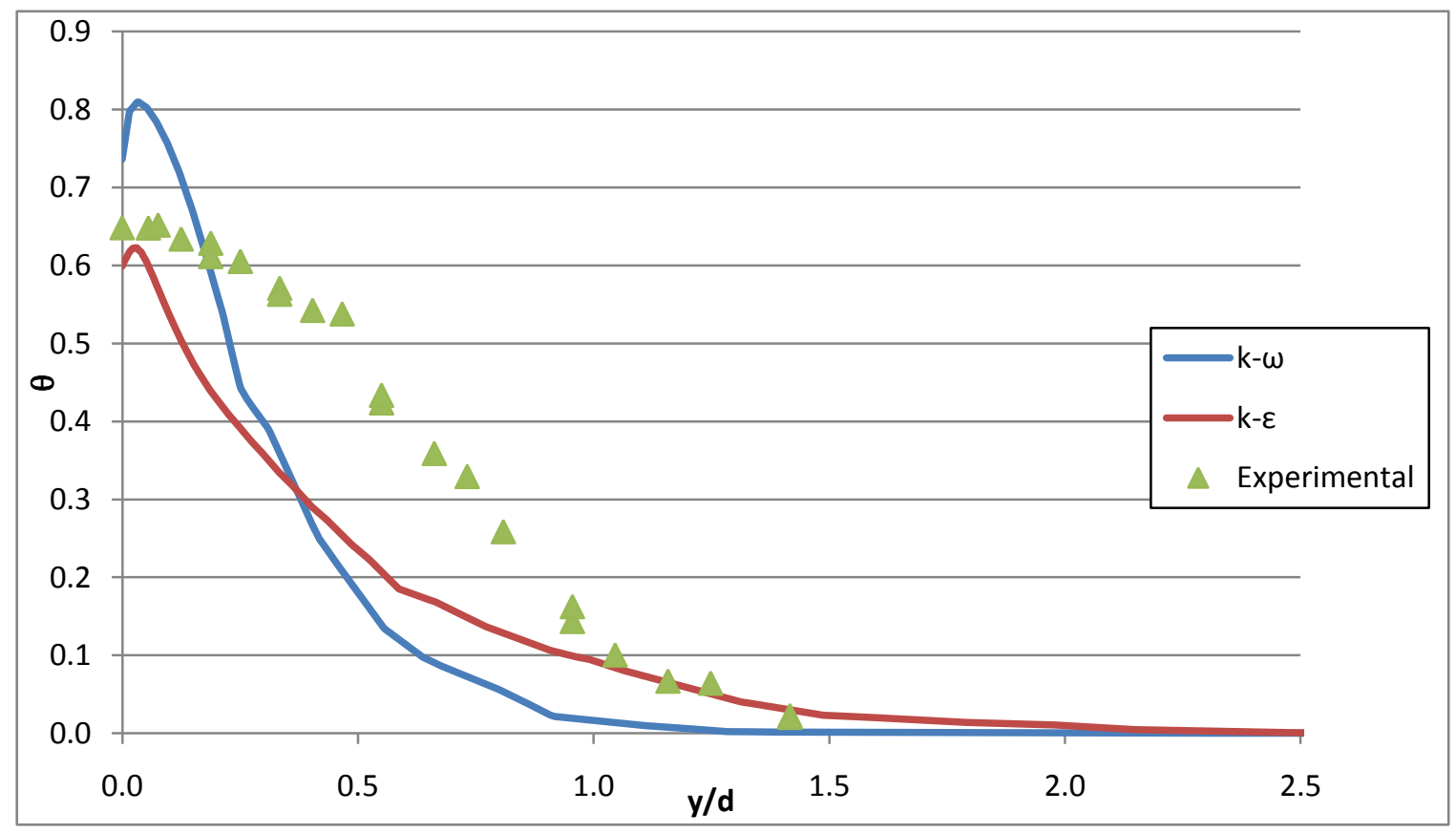

Figure 3.18- $\theta$ profile at $\mathrm{z} / \mathrm{d}=4.9$ and $\mathrm{x} / \mathrm{d}=5.1$

Figure 3.18 represents the coolant profile at the $\mathrm{z} / \mathrm{d}=4.9$. This span location represents the region of coldest temperature for the experimental data. The $\theta$ value of 0.63 close to 
the surface is similar to the $\mathrm{k}-\varepsilon$ simulation while the $\mathrm{k}-\omega$ simulation shows colder temperature close to the surface. The experimental data show mixing layer thickness similar to the k- $\varepsilon$ model while the $\mathrm{k}-\omega$ simulation shows smaller mixing layer thickness. Figure 3.18 shows that the 0.1 isotherm occurs a distance of $y / d=1$ for $k-\varepsilon$ while it occurs closer to the surface, at $y / d=0.6$ for the $k-\omega$. The pattern of the $k-\varepsilon$ simulation showing increased mixing with the mainstream is similar to the contour plots at the stagnation plane. The slopes of the $\mathrm{k}-\varepsilon$ and experimental temperature profiles are similar but the $\mathrm{k}-\varepsilon$ profile shows a concave shape while the experimental data is decreases more linearly.

The increased mixing of the coolant also be understood in terms of turbulent viscosity at the $\mathrm{x} / \mathrm{d}=5.1$ plane. Figure 3.19 reveals that the turbulence viscosity levels are significantly higher for $\mathrm{k}-\varepsilon$. 


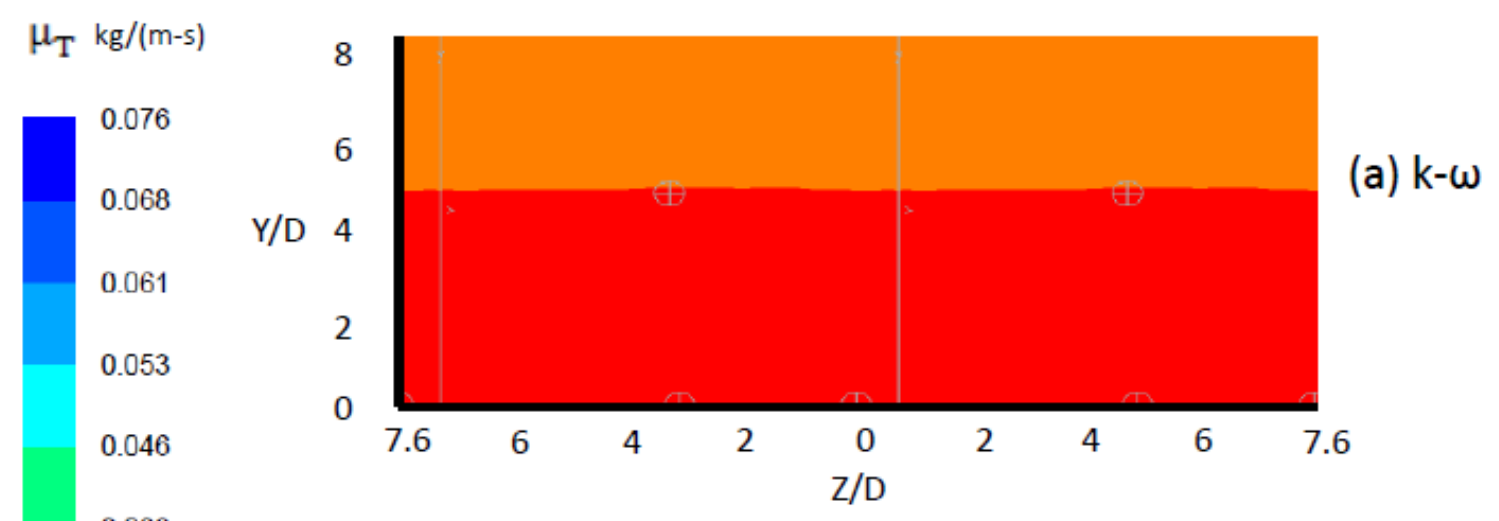

0.038

0.03

0.023

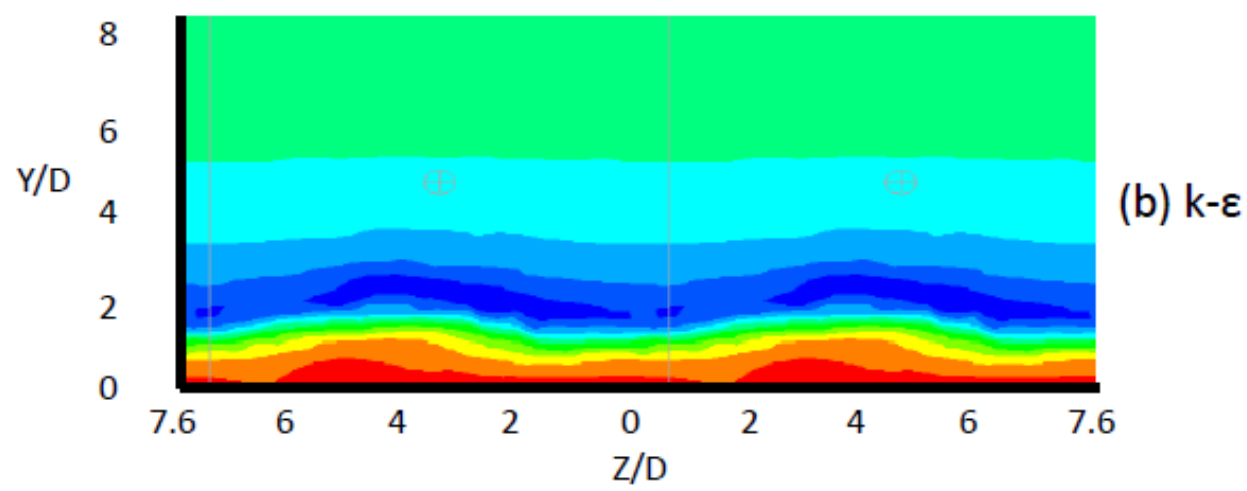

Figure 3.19- Contours of Turbulence Viscosity at $\mathrm{x} / \mathrm{d}=5.1$

As mentioned earlier, higher $\mu_{\mathrm{T}}$ levels translate to higher levels of Reynolds shear stress. The turbulence eddies have higher energy and diffuses more coolant into the mainstream. Thus, $\mathrm{k}-\varepsilon$ simulation has higher mixing levels. The pattern of the $\mathrm{k}-\varepsilon$ showing increased coolant mixing and increased $\mu_{\mathrm{T}}$ is similar to the stagnation plane and the $\mathrm{x} / \mathrm{d}=2$ plane. 


\subsection{Region of no coolant influence}

It is noted that the turbulence models show different coolant flow fields. While the k$\omega$ model captures near wall effects such as separation, the k- $\varepsilon$ model shows increased mixing of the coolant with the mainstream in regions away from the surface. Turbulent quantities such as $\mu_{\mathrm{T}}$ have been analyzed at different $\mathrm{x} / \mathrm{d}$ planes and the $\mathrm{k}-\varepsilon$ model consistently shows elevated levels. Even though the approach velocities of the freestream and the inlet freestream turbulences are identical for the models, the turbulence intensity levels are consistently higher for the $\mathrm{k}-\varepsilon$ simulations. Thus the exiting coolant experiences inherently different levels of turbulent kinetic energy and handle the freestream differently.

It is possible that the different turbulence levels are a result of the differences in coolant interaction with the mainstream and the subsequent treatment of shear flows by the turbulence models. In order to reduce the confounding effects of coolant flow, the temperature and velocity flowfields and turbulence levels were analyzed in a region of the model where the effect of coolant is minimal. Contour plots of the adiabatic model surface (Figure 3.1) shows that in $\mathrm{x} / \mathrm{d}=12.54$ ( 90 degree), there are regions where the surface temperature are close to mainstream temperature and thus experience minimal coolant presence. For the subsequent analysis, the conducting model was used and the spanwise location of lowest $\phi$ was found at the $\mathrm{x} / \mathrm{d}=12.54$ downstream location and off

the surface velocity and temperature fields are compared between turbulence models. 
At $\mathrm{x} / \mathrm{d}=12.54$, the lowest $\phi$ values is 0.267 for both turbulence models and spanwise location of lowest $\phi$ is $\mathrm{z} / \mathrm{d}=3.4$ and 2.6 from the stagnation exit hole lip for $\mathrm{k}-\omega$ and $\mathrm{k}-\varepsilon$ respectively. The location of lowest $\phi$ differs between models because the stagnation coolant experiences different lateral spreading downstream.

Figure 3.20 and 3.21 represent the velocity magnitude profiles at the location of lowest $\phi$. The distance $y / d=16$ from the model surface represents the top of the wind tunnel where a no shear boundary condition is imposed. With the no slip condition, the velocity is zero at the model surface. Both models show similar steep velocity gradients in the normal direction and this represents a turbulent boundary layer. While the profiles are the same for $y / d<0.05$ and for $y / d>1$, there is a distinct difference for $0.05<y / d<1$. In order to better understand the difference; the flowfield of the freestream air must be analyzed. With freestream air encountering the model, there is a region of stagnation at $\mathrm{x} / \mathrm{d}=0$ location. The flow configuration imposes a negative pressure gradient on the flow as it travels in the downstream direction. Thus, the freestream and coolant mixtures accelerates downstream of the stagnation line up until $\mathrm{x} / \mathrm{d}=12.54$ or the region of constant cross sectional area. The region of highest velocity magnitude shown in Figure 3.20 is result of the acceleration of the freestream as it travels downstream. But the extent of acceleration differs between models with the k- $\omega$ simulation having higher peak velocity. While the velocity goes to zero at the wall for a viscid flow with no slip conditions, the velocity of the flow would increase up until the wall for an invicid flow. Thus the decrease in the velocity profile when approaching the wall is a result of viscous forces. As shown in Figure 3.21 , the $k-\varepsilon$ velocity profile starts to decrease in value at $y / d=1$ but the $k-\omega$ 
shows a decrease in velocity profile only at $y / d=0.25$.Thus the viscous forces extend farther away from the wall for the k- $\varepsilon$. For $y / d>1$, both models are similar with the velocity profiles decreasing up until $\mathrm{y} / \mathrm{d}=12$ and then flattening out to a constant velocity of $20.4 \mathrm{~m} / \mathrm{s}$.

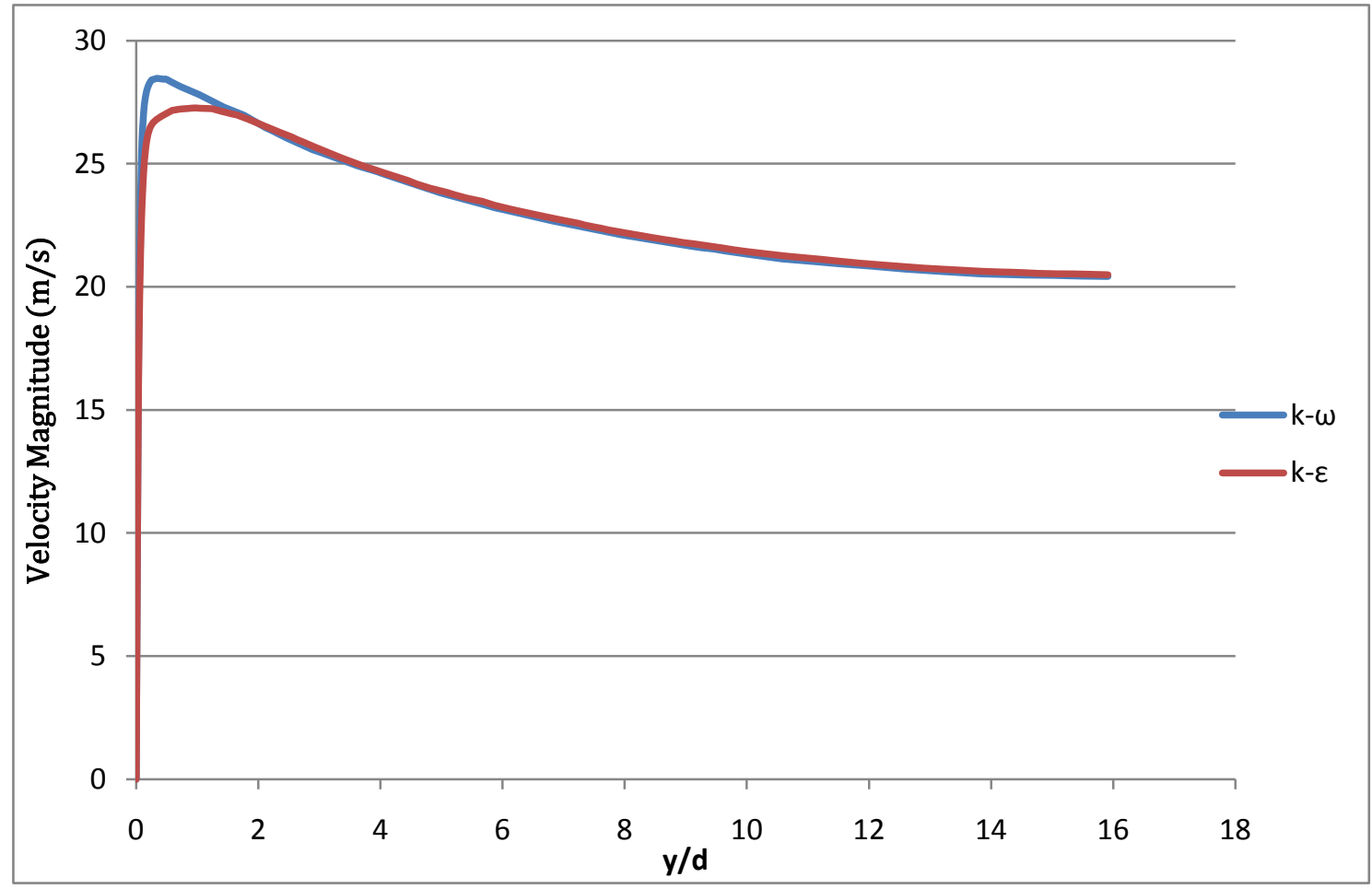

Figure 3.20- Velocity magnitude profile at location of lowest $\phi$ and $x / d=12.54$ 


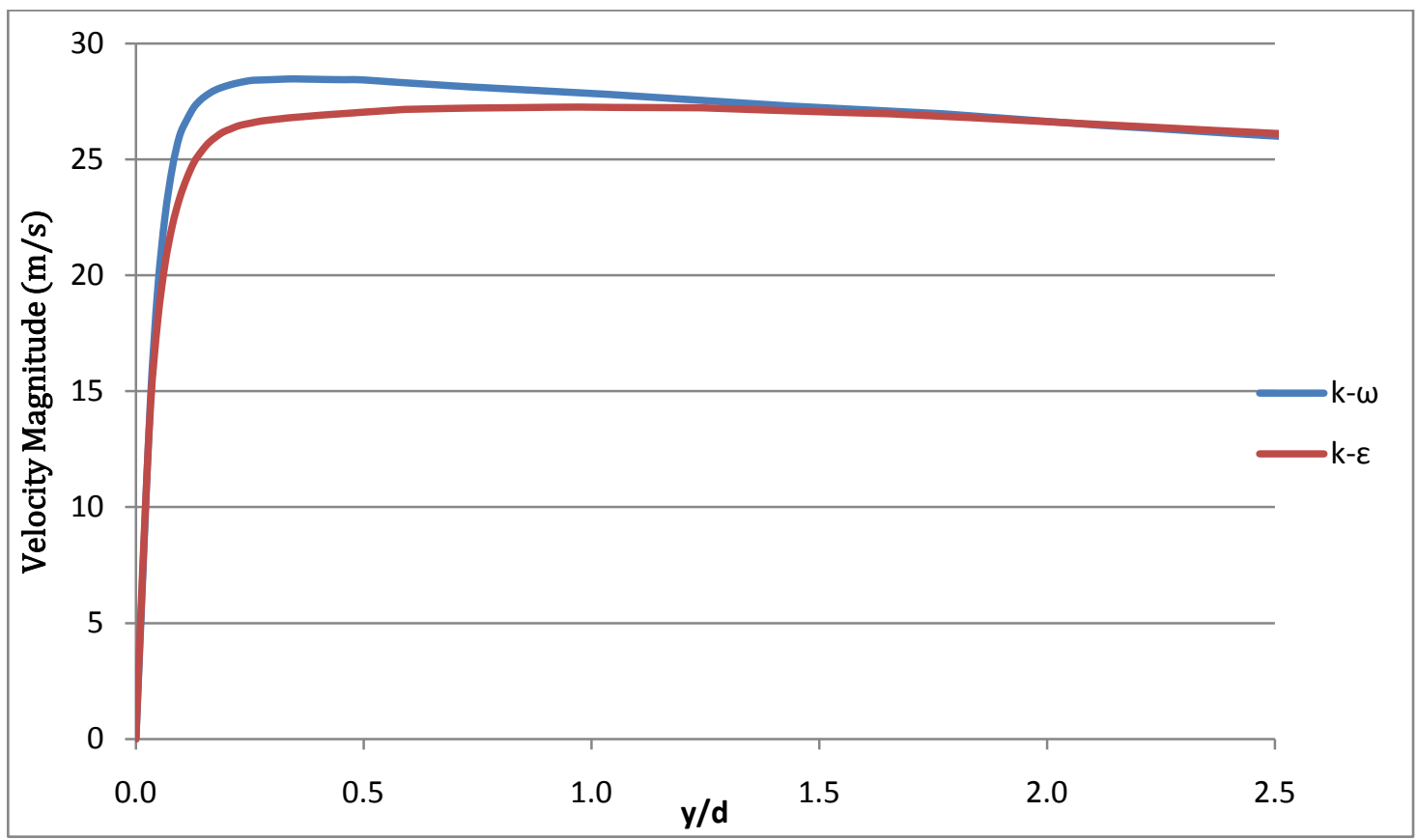

Figure 3.21-Velocity magnitude profile at location of lowest $\phi$ and $\mathrm{x} / \mathrm{d}=12.54$, near wall region

The temperature profiles off the surface at the region of lowest $\phi$ also differ between turbulence models, as shown in Figure 3.22. Both turbulence models have similar $\theta$ values near the surface, and both show a steep decrease in $\theta$ (increase in temperature) moving away from the wall. Since the viscous boundary layer effects appear to extend to $y / d=1$, the thermal boundary layer would not extend beyond $y / d>1$. Hence the non zero $\theta$ values above the surface is likely due to remnant coolant presence. The colder temperature at the surface is due to conduction through the solid and the gas temperature above the model matches the surface temperature. For $0.05<y / d<0.4$, the k$\omega$ simulations predicts lower $\theta$ values than the k- $\varepsilon$. And while the k- $\varepsilon$ shows a smooth decrease in $\theta$, the $\mathrm{k}-\omega$ profile shows a slight increase in $\theta$ values for $0.2<\mathrm{y} / \mathrm{d}<0.45$. Beyond $y / d=0.5$, the profiles for the turbulence models are similar. 


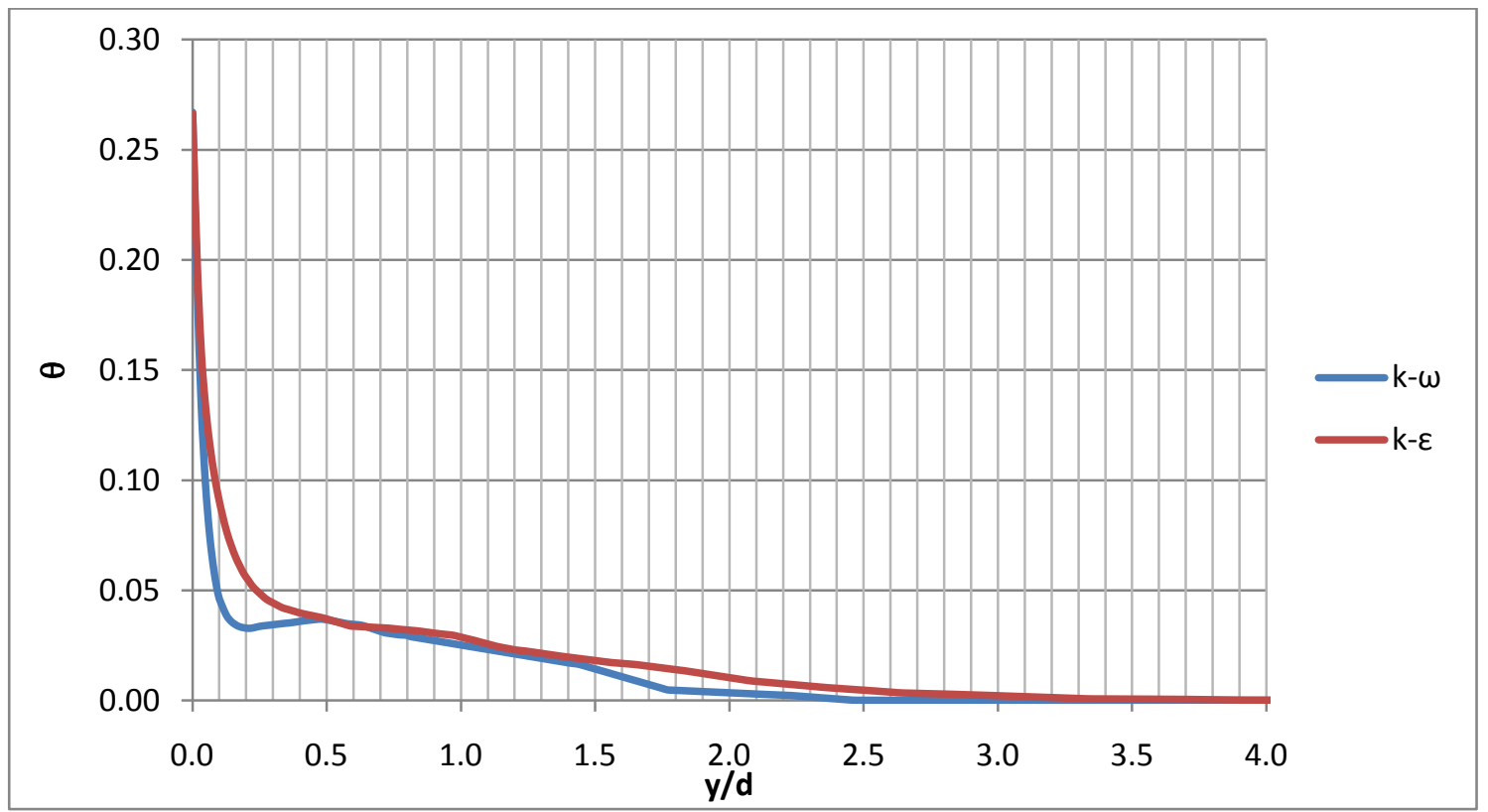

Figure 3.22- $\theta$ Profiles at $\mathrm{x} / \mathrm{d}=12.54$ and span location of lowest $\phi$

Figure 3.23 compares the turbulence intensity profiles for location of lowest $\eta$ at $\mathrm{x} / \mathrm{d}=12.54$. Similar to the stagnation plane, the $\mathrm{k}-\varepsilon$ model experiences higher levels of intensity, especially in the regions close to the surface. With higher intensities, the turbulent kinetic energy levels will be higher and thus resulting in greater turbulent diffusion for the k- $\varepsilon$ simulation. Further away from the surface, the turbulent intensity levels continue to decrease up to near the tunnel top. 


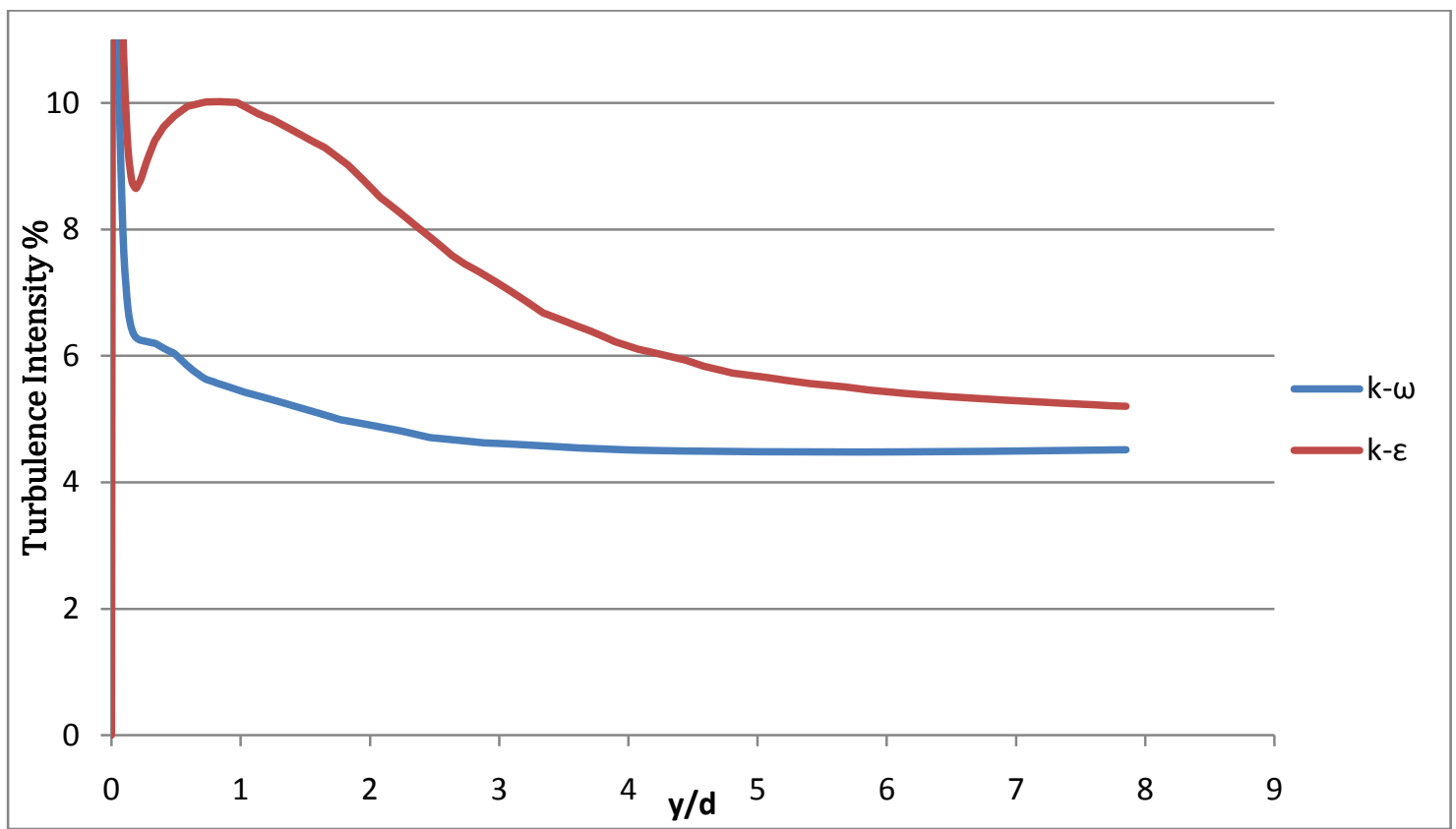

Figure 3.23- Turbulence Intensity Profiles at $\mathrm{x} / \mathrm{d}=12.54$ and span location of lowest $\phi$ 


\section{Chapter 4- Conclusion}

This chapter summarizes the key results and conclusions present in this thesis. Also, recommendations of future work are presented.

\section{1- Results and Conclusion}

The focus of this study was to evaluate CFD predictions of film cooling on a leading edge model by comparing it to experimental results. The leading edge model had three rows of film cooling holes, with one row on the stagnation line and two additional off stagnation rows at $\pm 25^{\circ}$. The thermal conductivity of the conducting model was specified such that the Biot number of the model matched that for a typical engine blade. The RKE and SST turbulence models were used in this study because these models showed superior performance in film cooling predictions compared to other RANS models. Comparison between the CFD predictions and experiments was done using adiabatic effectiveness and thermal field contours. All the analyses presented in this thesis were for $\mathrm{M}=2$ and $\mathrm{DR}=1.5$.

Contours of adiabatic effectiveness showed that the k- $\omega$ model predicted coolant separation at the stagnation plane while the k- $\varepsilon$ model predicted that the stagnation coolant remained attached to the model surface. The experimental adiabatic effectiveness and thermal field contours indicated stagnation coolant separation. The k- $\varepsilon$ predictions of laterally averaged adiabatic effectiveness $\eta$ were in very good agreement with the experimental measurements. Meanwhile, the k- $\omega$ predictions were distinctly higher than 
the experimental values throughout the length of the leading edge model. But, both CFD models overpredicted laterally averaged adiabatic effectiveness at the stagnation line.

The downstream spreading of the coolant also differs between CFD turbulence models. For the k- $\varepsilon$ case, coolant from the stagnation line holes turns in the downstream direction closer to the exit hole. Meanwhile, for the k- $\omega$ case coolant from the stagnation line holes preserves its momentum in the spanwise direction for farther distances away from the exit hole before moving downstream. The k- $\varepsilon$ stagnation coolant combines with the core of the offstagnation hole coolant closer to the exit of the hole and the combined jets move downstream in a narrow path. But the k- $\omega$ stagnation coolant reaches $5.1 \mathrm{~d}$ downstream of the stagnation line at a further spanwise distance from the off stagnation exit hole and thus the combined stagnation and offstagnation coolant jets are spread across a greater span length of the model. Experimental data shows stagnation coolant reaching the off stagnation row at a similar spanwise distance to the $\mathrm{k}-\omega$ prediction.

Analysis of the thermal field contours at the stagnation plane also showed differences in coolant diffusion into the mainstream. The diffusion of the coolant was compared using the $\theta=0.1$ isotherm distance. The $\mathrm{k}-\varepsilon$ prediction of stagnation coolant flow in the stagnation plane extended further away from the surface compared to the k- $\omega$ simulations but both CFD models showed similar trends, with a decrease in thickness from 1d downstream of the lip of the stagnation hole until the location of the adjacent stagnation coolant exit hole. But the experimental data shows the coolant extending further into the mainstream upon travelling in the spanwise direction. The growth in $\theta=0.1$ isotherm 
thickness continues up until the adjacent exit hole. Analysis of individual thermal profiles at the stagnation plane showed that near the coolant exit hole, before separation, both CFD models agreed reasonably well with the experimental profiles. But for $2 \mathrm{~d}$ downstream of the lip of the stagnation hole, the CFD predictions differed from the experimental thermal profile. Experimental profiles showed a constant $\theta$ value extending from the surface to beyond $0.5 \mathrm{~d}$, followed by a decrease in $\theta$ value but the CFD models showed highest $\theta$ value closer to the surface. By $4 \mathrm{~d}$ downstream of the stagnation hole, the experimental and CFD predictions differed significantly.

Thermal field contours were analyzed at 5.1d downstream of the stagnation line $(\mathrm{x} / \mathrm{d}=5.1)$ in order to study off stagnation coolant behavior. The general shape of the offstagnation coolant is similar for both CFD models and the experimental data, with the offstagnation coolant spreading a $\Delta \mathrm{z} / \mathrm{d}=3.3$ in the spanwise direction. But the core of the CFD offstagnation coolant jet is significantly colder than the experimental value. At $\mathrm{x} / \mathrm{d}=5.1$, both CFD models had significant regions of $\theta>0.8$ while the core of the experimental offstagnation coolant jet was warmer at $0.6<\theta<0.7$. The CFD models also predict more spreading of the off stagnation coolant in the $y / d$ direction compared to the experimental data.

The turbulent viscosity and intensity were checked along the stagnation plane and $\mathrm{x} / \mathrm{d}=5.1$ offstagnation plane for the CFD models. The $\mathrm{k}-\varepsilon$ simulation had significantly higher turbulent viscosity than the k- $\omega$ simulation at both locations. Also, the turbulent 
intensity level is higher for the $\mathrm{k}-\varepsilon$ simulation especially in the region of coolant and mainstream mixing.

\section{2- Recommendations for Future work}

While film cooling can lower the surface temperature of a model, it can also result in an augmentation of the heat transfer coefficient. An area of future research could be the comparison of heat transfer augmentation $\left(\mathrm{h}_{\mathrm{f}} / \mathrm{h}_{\mathrm{o}}\right)$ predictions between the RKE model and the SST k- $\omega$ turbulence models on a leading edge model. With overall effectiveness and heat transfer coefficient augmentation predictions, the net heat flux reduction due to film cooling can be analyzed.

Another area of research could be the analysis of the effects of freestream turbulence on the coolant flowfield for the CFD models. Both RKE and SST models under predicted diffusion of the coolant into the mainstream at $6 \%$ freestream turbulence. Increasing or decreasing the freestream turbulence could help explain some of the discrepancies between the CFD predictions.

An area of interest would be the investigation of the SST k- $\omega$ model regarding transition from k- $\omega$ to k- $\varepsilon$. The role of blending function used in the SST k- $\omega$ model could be investigated. Also for the k- $\omega$ grid, a sensitivity test could be performed by varying first cell and thickness of the outer prism layer 
Film cooling simulations could also be run on more advanced CFD models such as

LES and DNS. Such models could capture the unsteady effects and the anisotropy of the coolant flowfield. 


\section{Appendix - Low Freestream Turbulence}

The simulations for RKE model were also conducted at lower freestream turbulence levels of $1 \%$. Figure A. 1 shows the $\theta$ contours along the stagnation plane for high freestream turbulence of $6 \%$ and the low freestream turbulence of $1 \%$. Both simulations show similar coolant flow upon exiting the stagnation coolant hole. There is no coolant separation for either simulation. But the diffusion of coolant into the mainstream is different. For $\mathrm{z} / \mathrm{d}>2$, the low freestream turbulence case shows coolant extending further away from the surface than the higher freestream turbulence case.

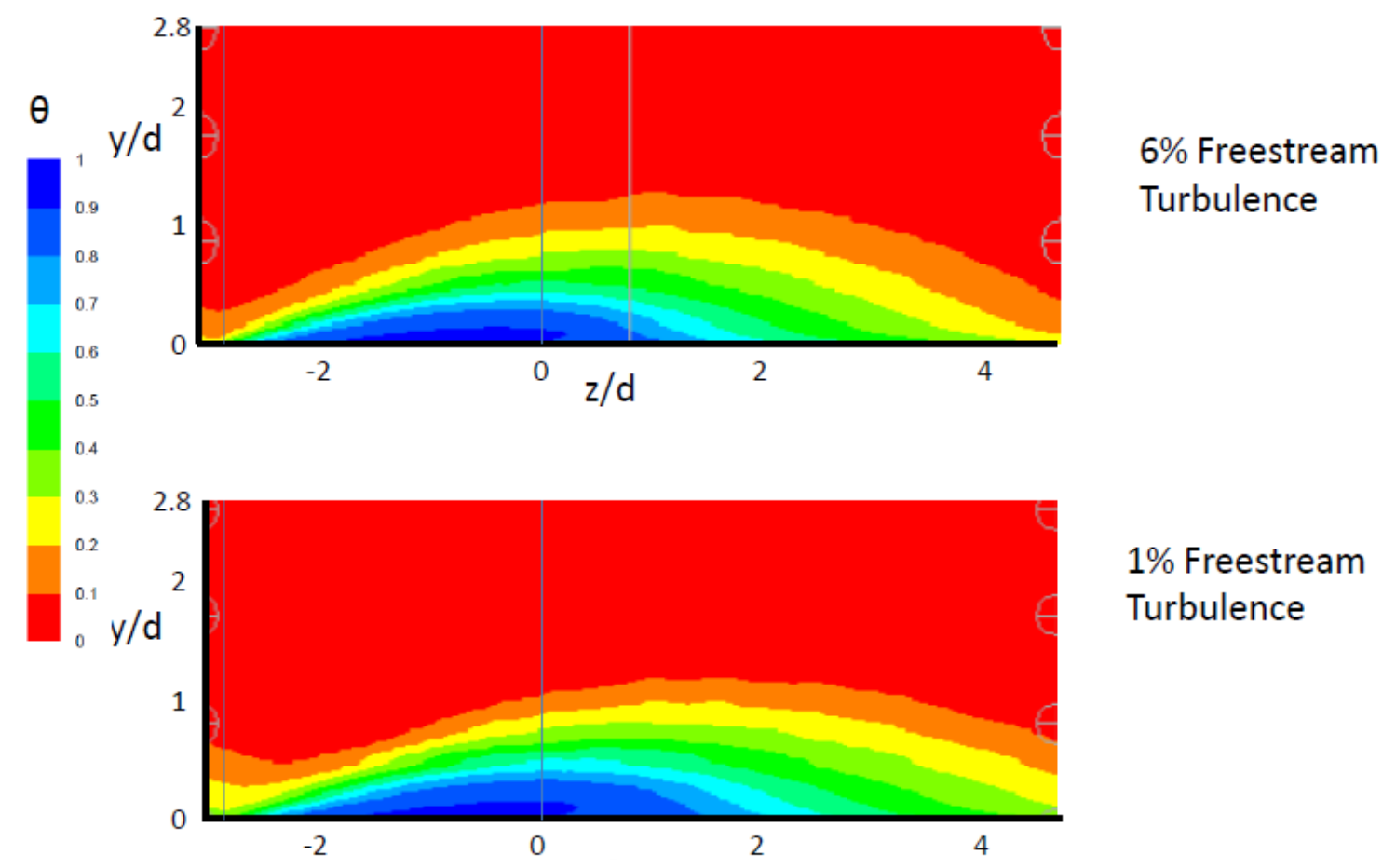

Figure A.1- Contours of $\theta$ along the stagnation plane with varying freestream turbulence 
Figure A.2 also shows $\phi$ contours for the high and low freestream turbulence simulations. Along the stagnation plane, the high freestream turbulence simulations predicts highest $\phi$ values closer to the exit of the hole with $\phi$ values decreasing with spanwise distance away from the exit of the hole. But, along the stagnation line, the low freestream turbulence shows highest $\phi$ value further away from the exit of hole. Such a $\phi$ distribution would suggest separation but Figure A.1 shows that the stagnation coolant does not separate for the low turbulence simulation. Also the low freestream turbulence simulations shows higher $\phi$ values extending further away from the exit of the stagnation hole even at downstream locations. Thus, the low freestream turbulence stagnation coolant retains more momentum and colder temperatures further away from the stagnation exit hole than the high freestream turbulence case.

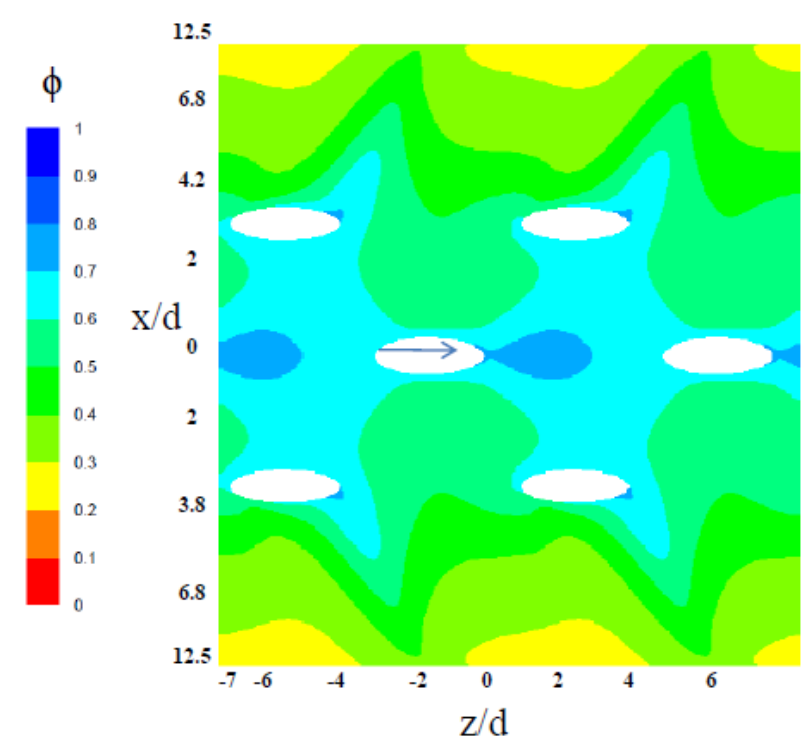

$6 \%$ Freestream

Turbulence

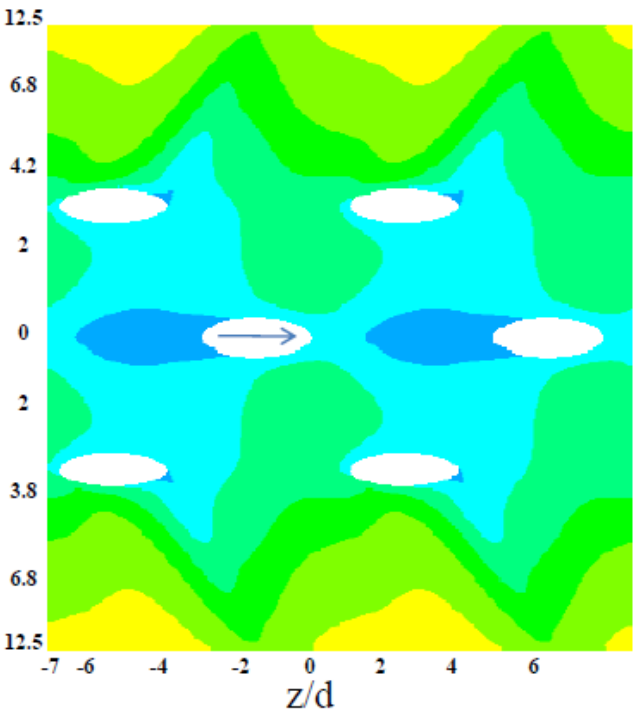

$1 \%$ Freestream Turbulence

Figure A.2- Contours of overall effectiveness with varying freestream turbulence 


\section{References}

Albert, J.E., 2003, "Adiabatic and overall effectiveness measurements of a forward diffused film cooling configuration for turbine blade leading edge model", M.S. Thesis, The University of Texas, Austin, Texas.

Albert, J.E., Bogard, D. G., Cunha, 2004, "Adiabatic and overall effectiveness for a film cooled blade", Proceedings of the ASME Turbo Expo, 2005, GT2004-53998

Coulthard, S.M., Volino, R.J., Flack, K. A., 2007, "Effect of jet pulsing on film cooling Part I: Effectiveness and flow-field temperature results", Journal of Turbomachinery, Vol. 129, n 2, p 232-246

Cruse, M. W., Yuki, U.M., Bogard, D.G., 1997, "Investigation of various parametric influences on leading edge film cooling”, ASME Turbo Expo 1997 ,97-GT-296

Cutbirth, J. M., Bogard, D. G., 2002, "Thermal field and flow visualization within the stagnation region of a film-cooled turbine vane", Journal of Turbomachinery, Vol. 124, n 2, p 200-206

Dyson, T. E., Bogard, D. G., Piggush, J.D., Kohli, A., 2010, “Overall effectiveness for a film cooled turbine blade leading edge with varying hole pitch", ASME Paper ,GT201023707

Fluent Manual, 2009, Version 6.3.26, Fluent Inc., Lebanon, New Hampshire

Harrison, K. and Bogard, D. G., 2008, “Comparison of RANS turbulence models for prediction of film cooling performance”, ASME Paper GT 2008-51423

Harrison, K. and Bogard, D. G., 2008, "Evaluation of the Use of the Adiabatic Wall Temperature to Predict Heat Fluxes for Film Cooled Turbine Airfoils", ISROMAC 122008-20187.

Johnston, C. A., 1999, "High freestream turbulence effects on film cooling of a turbine blade leading edge", M.S Thesis, University of Texas, Austin, Texas

Keimasi, M.R., Taeibi-Rahni, M., 2001, "Numerical simulation of jets in a crossflow using different turbulence models", AIAA Journal, Vol. 39, n 12, p 2268-2277

Kovzulovic, D. and Rober, T., 2006, Contribution of Turbulence Equation Terms to the Shear Stress Balance, Proc. of the Fourth International Conference on Computational Fluid Dynamics, July 2006, p 511-516 
Lin, Y.-L and Shih, T.I-P., 2001, "Film Cooling of a Cylindrical Leading Edge with Injection Through Rows of Compound Angle Holes", Journal Of Heat Transfer, Vol.123, n 4, pp. 645-654

Laskowski, G. M., Tolpadi, A. K., and Ostrowski, M. C., 2007, "Heat Transfer Predictions of Film Cooled Stationary Turbine Airfoils", ASME Paper No. GT200727497

Maikell, J.S., 2008, "Experimental Study of the effect of TBC and angle of attack on overall cooling performance on a leading edge with one and three rows of cooling holes" , M.S. Thesis, The University of Texas, Austin, Texas.

Medic, G., and Durbin P. A., 2002, “Towards improved prediction of heat transfer in turbine blades", Journal of Turbomachinery, Vol. 124 pp.187-192.

Mills, A. F., 1999, Heat Transfer, Second edition, Prentice Hall, Inc.

Mouzon, B.D., Terrell, E.J., Albert, J.E., Bogard, D.G., 2005. "Net heat flux reduction and overall effectiveness for a turbine blade leading edge". ASME Paper No. GT200569002

Pope, S.B., 2000, Turbulent Flows, Cambridge University Press

Ravelli, S., Dobrowolski, L., Bogard, D.G., 2010, "Evaluating the Effect of Internal Impingement Cooling on a Film Cooled Turbine Blade Leading Edge", ASME Paper No. GT2010-23002

Ravelli, S., 2009, Private Communications

Shih, T.-H., Liou,W, Shabbir, A., Yang, Z., Zhu, J., 1995, “A new k- $\varepsilon$ Eddy Viscosity Model For High Reynolds Number Turbulent Flows”, Computers Fluids, Vol.24, n 3, pp. 227-238

Teng. S, Han, J.C., Poinsatte, P. E., 2001 , "Effect of Film-Hole Shape on Turbine-Blade Film-Cooling Performance", Journal of Thermophysics and Heat Transfer, Vol. 15, n 3, p 257-265

Terrell, E. J, Mouzon, B. D., Bogard, D. G., 2004, “Convective Heat Transfer Through Film Cooling Holes of a Gas Turbine Blade Leading Edge", ASME Paper, No. GT200569003

Terrell, E. J, 2004, “A Study of the Convective Heat Transfer through Film Cooling Holes of a Gas Turbine Blade Leading Edge", M.S. Thesis, The University of Texas, Austin, Texas.

York, W. D. and Leylek, J.H., 2000, "Impact of Film Cooling Jets on Turbine Aerodynamics Losses”, Journal of Turbomachinery, Vol. 122, p 537-545 
York, W. D. and Leylek, J.H., 2002, "Leading-Edge Film Cooling Physics: Part IAdiabatic Effectiveness", ASME Paper No. GT-2002-30166 


\section{Vita}

Sibi Mathew was born on April 10 $0^{\text {th }}, 1986$ in Kuwait, the son of Ani Alexander and K.D Mathew. He grew up in Kuwait. Sibi graduated from Texas Tech University, Lubbock in May 2008 with a Bachelor of Science degree in Mechanical Engineering and a Minor in Mathematics. He graduated Summa Cum Laude with Honors Studies. During the summer of 2008, Sibi interned with Cameron, Houston as a subsea drilling engineer. He began his Master of Science in Mechanical Engineering at the University of Texas at Austin in August 2008. Sibi will graduate in August 2010 from the University of Texas at Austin.

Permanent Address- $4202,95^{\text {th }} \mathrm{St}$

Lubbock, TX 78741

This thesis was typed by the author 